\title{
Thermodynamic properties of sea air
}

\author{
R. Feistel ${ }^{1}$, D. G. Wright ${ }^{2}$, H.-J. Kretzschmar ${ }^{3}$, E. Hagen ${ }^{1}$, S. Herrmann ${ }^{3}$, and R. Span ${ }^{4}$ \\ ${ }^{1}$ Leibniz Institute for Baltic Sea Research, 18119 Warnemünde, Germany \\ ${ }^{2}$ Bedford Institute of Oceanography, Dartmouth, NS, Canada \\ ${ }^{3}$ Department of Technical Thermodynamics, Zittau/Görlitz University of Applied Sciences, 02763 Zittau, Germany \\ ${ }^{4}$ Lehrstuhl für Thermodynamik, Ruhr-Universität Bochum, 44780 Bochum, Germany
}

Received: 6 August 2009 - Published in Ocean Sci. Discuss.: 13 October 2009

Revised: 4 January 2010 - Accepted: 13 January 2010 - Published: 1 February 2010

\begin{abstract}
Very accurate thermodynamic potential functions are available for fluid water, ice, seawater and humid air covering wide ranges of temperature and pressure conditions. They permit the consistent computation of all equilibrium properties as, for example, required for coupled atmosphereocean models or the analysis of observational or experimental data. With the exception of humid air, these potential functions are already formulated as international standards released by the International Association for the Properties of Water and Steam (IAPWS), and have been adopted in 2009 for oceanography by IOC/UNESCO.

In this paper, we derive a collection of formulas for important quantities expressed in terms of the thermodynamic potentials, valid for typical phase transitions and composite systems of humid air and water/ice/seawater. Particular attention is given to equilibria between seawater and humid air, referred to as "sea air" here. In a related initiative, these formulas will soon be implemented in a source-code library for easy practical use. The library is primarily aimed at oceanographic applications but will be relevant to air-sea interaction and meteorology as well.

The formulas provided are valid for any consistent set of suitable thermodynamic potential functions. Here we adopt potential functions from previous publications in which they are constructed from theoretical laws and empirical data; they are briefly summarized in the appendix. The formulas make use of the full accuracy of these thermodynamic potentials, without additional approximations or empirical coefficients. They are expressed in the temperature scale ITS-90 and the 2008 Reference-Composition Salinity Scale.
\end{abstract}

\section{Correspondence to: $\mathrm{R}$. Feistel (rainer.feistel@io-warnemuende.de)}

\section{Introduction}

In meteorology and oceanography, many standard textbooks present the thermodynamic properties of moist air, seawater or ice in the form of a collection of independently determined correlation equations for selected quantities (e.g., Gill, 1984; Emanuel, 1994; Seinfeld and Pandis, 1998; Millero, 2001; Jacobson, 2005) or by means of meteorological charts such as the Stüve diagram, the emagram or the tephigram (Rogers and Yau, 1989). Often, some of these equations rely on certain simplifications (e.g., ideal gas, constant heat capacity, constant latent heat); their uncertainty, completeness, mutual consistency or range of validity is not always clearly stated. The particular selection chosen may depend on personal preferences of the author over various alternative empirical formulas available from the scientific literature. Although the air-sea interface forms the largest contribution to the atmospheric boundary layer, meteorological equations which account for the ocean's salinity are comparatively scarce and apparently considered to be of marginal interest. Explicit formulas for fundamental quantities such as entropy are often missing (McDougall and Feistel, 2003; Pauluis et al., 2008). Even if mathematical expressions for enthalpy, entropy or chemical potential are available, without an explicit specification of their freely adjustable constants it is difficult to compare the results from different formulas or to consistently combine one with another (Feistel et al., 2008b).

An alternative, systematic, theoretically more elegant and satisfactory concept is the construction and evaluation of thermodynamic potentials, as demonstrated by IAPWS ${ }^{1}$ in the form of its releases on fluid water (IAPWS, 2009a), ice (IAPWS, 2009b) and seawater (IAPWS, 2008). On this

\footnotetext{
${ }^{1}$ IAPWS: The International Association for the Properties of Water and Steam, http://www.iapws.org.
} 
basis, together with IAPWS, the $\mathrm{SCOR}^{2} / \mathrm{IAPSO}^{3}$ Working Group 127 (WG127) on Thermodynamics and Equation of State of Seawater in cooperation with UNESCO-IOC ${ }^{4}$ developed a proposal for a new international standard for oceanography, TEOS- $10^{5}$ (IOC, 2010), which has replaced the currently valid 1980 International Equation of State of Seawater (EOS-80, Fofonoff and Millard, 1983) after almost three decades without formal updates to account for progress in this field (Feistel, 2008a, b; Feistel et al., 2008b; IAPWS, 2008a; IOC, 2009).

All available accurate experimental thermodynamic data for a given substance can be suitably combined with each other for the construction of a single mathematical function, the thermodynamic potential (Gibbs, 1873; Fofonoff, 1962; Feistel, 1993; Pruppacher and Klett, 1997; TillnerRoth, 1998; Alberty, 2001; Hantel and Mayer, 2006; Aleksandrov and Orlov, 2007; Feistel, 2008a; Herrmann et al., 2009; IOC, 2010), from which all thermodynamic properties can be derived analytically or numerically. Moreover, if the potential functions of different substances obey certain mutual consistency requirements (Feistel et al., 2008b), any mixtures, phase equilibria or composite systems can additionally be described this way by rigorous thermodynamic methods, i.e. by appropriate algebraic combinations of partial derivatives of the potential functions.

For practical use in oceanography and meteorology, Gibbs functions $g(T, P)$ are the most convenient potentials because they provide all properties as functions of temperature $T$ and pressure $P$ which are directly available from measurements (Tillner-Roth, 1998). If the range of validity includes a phase transition region, Gibbs functions possess multiple values in the vicinity of the phase transition line in the $T-P$ diagram (Kittel, 1969; Stanley, 1971). Helmholtz functions $f(T, \rho)$ depending on temperature and density $\rho$ are single-valued even in this case and are therefore the preferred numerical formulation in such regions of parameter space. Enthalpy $h(s, P)$ as a function of entropy $s$ and pressure is particularly useful for the description of adiabatic (isentropic) processes (Feistel and Hagen, 1995; McDougall, 2003; Pauluis et al., 2003; Feistel, 2008a), e.g. for the computation of potential temperature (v. Helmholtz, 1888; v. Bezold, 1888; HellandHansen, 1912; Rogers and Yau, 1996; McDougall and Feistel, 2003). All these potential functions are mathematically and physically equivalent; the choice of which to use depends on application requirements or numerical simplicity.

\footnotetext{
${ }^{2}$ SCOR: Scientific Committee on Oceanic Research, http:// www.scor-int.org.

${ }^{3}$ IAPSO: International Association for the Physical Sciences of the Ocean, http://iapso.sweweb.net.

${ }^{4}$ IOC: Intergovernmental Oceanographic Commission, http:// ioc-unesco.org

${ }^{5}$ TEOS-10: International Thermodynamic Equation of Seawater 2010, http://www.teos-10.org.
}

The short term "sea air" is used in this paper to refer to systems composed of humid air and seawater, freshwater or ice in mutual thermodynamic equilibrium, e.g. modelling properties of a system including the ocean surface mixed layer and the marine atmospheric boundary layer. In addition to the three thermodynamic potentials for fluid water, ice and seawater endorsed by IAPWS, potential functions for dry air and virial coefficients for air-vapour interaction are required for the construction of a potential function for humid air. These formulas are available from the literature as discussed later and are used here to construct the desired potential function. An IAPWS document on this formulation is in preparation (IAPWS, 2010). Combined, these four potentials permit the computation of thermodynamic properties of the atmosphere, the ocean, clouds, ice and lakes in a comprehensive and consistent manner, valid over wide ranges in temperature, pressure and concentrations, from polar cirrus clouds at high altitudes to saline estuaries in the tropics, with the highest accuracy presently available. Rather than being a mere theoretical exercise, these four independent functions and numerous properties derived thereof are available from a numerical source-code library which has currently been developed, supporting the implementation of the intended new oceanographic TEOS-10 standard (Feistel et al., 2009; Wright et al., 2009). The library will be available in Fortran and Visual Basic/Excel for easy use on various platforms. Later, versions in Matlab and $\mathrm{C} / \mathrm{C}++$ are also planned.

The mathematical forms of the four thermodynamic potentials are briefly described in the appendix. In Sects. 3 to 9 , exploiting those potentials, mathematical formulas and relations are given for the computation of properties of humid air and its equilibria with liquid water, ice and seawater. These formulas are rigorous thermodynamic relations; they will remain valid even if the particular potential functions are updated in the future with new numerical coefficients or different algebraic structures.

The flux of water vapour from the oceans into the air is the main source of moisture for the atmospheric branch of the hydrological cycle, in which the moisture is carried by large-scale atmospheric circulation patterns. Via precipitation, a great part returns directly to the ocean or indirectly as riverine run-off from the continents. Spatial patterns of evaporation, precipitation, river discharge and overall runoff vary substantially over time, and the oceanic circulation adjusts to close the freshwater cycle on the global scale. The global freshwater balance controls the distribution of salinity which, via internal pressure gradients, constitutes a main driving factor for the circulation of the world ocean. Regional exchange processes at the ocean-atmosphere interface depend essentially on the redistribution of thermohaline water properties by oceanic currents. As sufficiently advanced global coupled ocean-atmosphere models become available, the remotely observed sea-surface temperature (SST) is being treated as an internal system variable rather than being formulated as a boundary condition of either an atmospheric 
or an oceanic climate model; it is thus becoming one of the most useful and sensitive indicators for the correctness of such models. Fully coupled atmosphere-ocean general circulation and ice-sheet models are presently the most important tools to understand the dramatic climatic impact on atmospheric water content, cloud formation, ice cover and sea level in the past and future (Trenberth et al., 2001; Lunt et al., 2008). The feedback associated with changes in atmospheric water content is a key process that must be accounted for in predictions of global warming (Willett et al., 2007; Dressler et al., 2008).

A consistent description of the fluxes of seawater vapour and latent heat, which continually pass across the oceanatmosphere interface on different spatial and temporal scales, is indispensable for models dealing with the coupled oceanatmosphere system. All thermodynamic quantities associated with such fluxes must be formulated consistently on both sides of the sea surface. While transport properties themselves cannot directly be computed from the equilibrium properties summarized in this paper, the deviation of measured or modelled conditions from those equilibrium properties represent the system's distance from equilibrium and estimate the thermodynamic Onsager forces (Glansdorff and Prigogine, 1971; De Groot and Mazur, 1984) driving the irreversible fluxes. Consequently, standard formulas which use measurable parameters to estimate the physical fluxes of energy, heat, water, or salt at the sea surface are based on equilibrium properties of seawater and humid air (Stull, 2003; Weller et al., 2008). For example, the corresponding bulk formulas for the ocean-atmosphere latent heat flux (Weare et al., 1981; Baosen, 1989; Wells and King-Hele, 1990) are more correctly expressed in terms of the sea-air specific humidity at the condensation point (Sects. 6, 12) rather than of saturated humid air. Measurements of the evaporation rate of seawater at different salinities were made recently by Panin and Brezgunov (2007).

In Sect. 2, with regard to ocean-atmosphere interaction, we first briefly review several vapour-pressure formulas and equations for humid air. In Sect. 3, the IAPWS Release 2008 for seawater is explained. Some recent formulations for dry air are discussed in Sect. 4. The generalisation for humid air follows in Sect. 5. The composite system of humid air in equilibrium with seawater (sea air) is analyzed in Sect. 6. Its special case of vanishing salinity, i.e. the equilibrium between pure water and saturated air is described in Sect. 7, in particular the saturation of air with respect to liquid water. Below the freezing point of seawater, ice appears in sea air as a third phase; in Sect. 8 extended formulas are discussed that account for the presence of ice, and in Sect. 9 we consider the related zero-salinity limit, "ice air". In Sect. 10 we discuss different definitions of relative humidity and derive its equilibrium value for the sea surface. Introducing enthalpy as a potential function in Sect. 11, we consider the properties of a well-mixed, isentropic atmosphere in local equilibrium with the sea surface. In the discussion, Sect. 12, we compare equilibrium properties of sea air with some observations of the marine atmosphere. The appendix summarizes the details of the different thermodynamic potential functions, formulas for properties derived thereof, conversion tables between the partial derivatives of different potentials and, for reference, simplified potential functions in the ideal-gas limit. Note that this limit implies pressure-independent but not necessarily temperature-independent heat capacities (Landau and Lifschitz, 1987). For the humid-air potential that is published in this form for the first time in this paper, tables with numerical check values are provided for the verification of the correctness of its implementations or modifications.

To use an approach and nomenclature that are consistent with oceanographic practice, some equations developed here differ slightly from common meteorological practice. Pure water serves as the general reference substance, in the ocean in liquid form, in the atmosphere in its vapour phase. Dissolved in or mixed with this fluid water are sea salt and dry air, both being natural mixtures of various chemical species, described by the absolute salinity, $S_{\mathrm{A}}$, as the mass fraction of salt in seawater, and the mass fraction of dry air, $A$, in humid air, including the limiting cases of freshwater $\left(S_{\mathrm{A}}=0\right)$, dry air $(A=1)$ or air-free vapour $(A=0)$.

The dissolution of air in water or seawater is always neglected in this paper. Also, problems related to metastable states, supersaturation or nucleation processes are not considered. The fallout of condensed water or ice from the real atmosphere depends on certain conditions such as the droplet size distribution. This precipitation problem is excluded from our theoretical model; in this paper we consider thermodynamic phase equilibria of gravity-free composite systems regardless of the shape of the phase boundaries.

The chemical composition of sea-salt, Table A1, is considered as constant, as described by Millero et al. (2008). The chemical composition of air, Table A2, is assumed to be fixed as described by Picard et al. (2008), although only the permanent constituents of air are considered here except for the variable water vapour content. The symbol for pressures is $P$ with corresponding superscripts. Thus, for example, we use $P^{\mathrm{V}}$ for the partial pressure of water vapour rather than $e$ as often used in meteorological texts. The symbol $s$ is used for the specific entropy rather than $\phi$ (as in the word "tephigram" which refers to the $T$ - $\phi$ diagram, where $\phi$ refers to specific entropy). Consistent with oceanography, the adiabatic lapse rate $\Gamma$ is defined here as the isentropic temperature change with respect to pressure rather than with respect to altitude, $\Gamma=(\partial T / \partial P)_{s}$, thus being immediately available from e.g. the related Gibbs function as an intrinsic thermodynamic property of air, independent of non-thermodynamic parameters such as gravity or distance.

Regarding the terminology of adiabatic processes and lapse rates, consistent with Pruppacher and Klett (1997), we refer to the lapse rate of dry air as "dry adiabatic", of unsaturated humid air as "moist adiabatic", of wet air (parcels containing humid air and liquid water) as "wet adiabatic" and 
of ice air (parcels containing humid air and solid ice) as "ice adiabatic". This is in contrast to textbooks such as Jacobson (2005) which, to some extent confusingly, link "dry" adiabatic lapse rates to unsaturated moist air, and "moist" adiabatic lapse rates to saturated parcels which condense water and warm up due to latent heat release during uplift.

If not explicitly mentioned otherwise, all equations are expressed in the temperature scale ITS- $90^{6}$ (Preston-Thomas, 1990) and in the new oceanographic Reference-Composition Salinity Scale (Millero et al., 2008; IOC, 2010) in $\mathrm{g} \mathrm{kg}^{-1}$ rather than in Practical Salinity on the 1978 scale. As usual in meteorology, in several figures the absolute pressure is given in units of $\mathrm{hPa}=100 \mathrm{~Pa}$.

\section{Available formulations for dry air, humid air, and the vapour pressure over water, seawater, or ice}

Standard textbooks (Gill, 1983; Jacobson, 2005) provide sets of various separate correlation equations for relevant thermodynamic properties of the atmosphere such as the heat capacity, the lapse rate or the latent heat. The estimated uncertainties in the proposed ideal-gas formulations for the density of dry and humid air are less than $0.2 \%$ under typical atmospheric temperature and pressure conditions (Jacobson, 2005).

A Helmholtz function for dry air was published by Lemmon et al. (2000), representing experimental data in the range 0-70 MPa and $60-873 \mathrm{~K}$ (reasonably predicting properties even up to $2000 \mathrm{MPa}$ and $2000 \mathrm{~K}$ ) as an extended revision of the previous equation by Jacobsen et al. (1990), completing it with the ideal-gas contributions for the major air constituents. The most recent ideal-gas heat capacities are available from Helmholtz function formulations for Nitrogen (Span et al., 2000, quantum rigid rotor model for 20-500 K with $0.01 \%$ uncertainty), Oxygen (Stewart et al., 1991, from experimental data at $30-3000 \mathrm{~K}$ with a maximum deviation of $0.003 \mathrm{~J} \mathrm{~mol}^{-1} \mathrm{~K}^{-1}$ ) and Argon (Tegeler et al., 1999, using the constant isobaric heat capacity $c_{P}^{\mathrm{Ar}, \mathrm{id}}=2.5 R$ with an uncertainty below $0.01 \%$ up to $10000 \mathrm{~K}$ ). Another equation for oxygen is available from Wagner and de Reuck (1987). We choose to use the more recent formulation of Lemmon et al. (2000) which combines the properties of the different air constituents including their ideal-gas parts, and is expressed on the ITS-90 scale. Experimental data for the heat capacity of air were published by Henry (1931) at atmospheric pressure for the temperature range $20-370^{\circ} \mathrm{C}$, and by Magee (1994) for 67-300 K at pressures up to $35 \mathrm{MPa}$. The different formulas and data available are reviewed in great detail by Lemmon et al. (2000).

\footnotetext{
${ }^{6}$ ITS-90: International Temperature Scale of 1990, www.bipm. org/en/publications/its-90.html.
}

The most recent and most accurate equation of state for moist air near atmospheric pressure is the CIPM ${ }^{7}-2007$ formula (Picard et al., 2008), replacing its predecessor known as the CIPM-81/91 equation (Giacomo, 1982; Davis, 1992) in particular for its corrected value of the Argon fraction. CIPM-2007 is valid in the range 0.06-0.11 MPa and 15$27^{\circ} \mathrm{C}$ for relative humidity $0-100 \%$. Its relative combined standard uncertainty in density is estimated to be $22 \mathrm{ppm}$ $\left(10^{-6}\right)$. The formulation of Hyland and Wexler (1983b) covers dry air at $173.15-473.15 \mathrm{~K}$ and humid air at $173.15-$ $372.15 \mathrm{~K}$ at pressures up to $5 \mathrm{MPa}$. For an accurate construction of humid-air formulations from separate equations for dry air and for water vapour, Harvey and Huang (2007) recalculated the second virial coefficient of $\mathrm{H}_{2} \mathrm{O}$-air interaction by evaluating the statistical configuration integral. Earlier second and also third virial coefficients for $\mathrm{H}_{2} \mathrm{O}$-air-air and $\mathrm{H}_{2} \mathrm{O}-\mathrm{H}_{2} \mathrm{O}$-air three-particle interactions are available from Hyland and Wexler (1983b). In this paper we use these second and third cross-virial coefficients in combination with Helmholtz functions for dry air from Lemmon et al. (2000) and for water vapour from IAPWS-95 (Appendix D; IAPWS, 2010).

Thermodynamic properties of water vapour including airfree saturation conditions are available from the IAPWS-95 formulation in the range $130 \mathrm{~K}-1273 \mathrm{~K}$ and pressures up to $1000 \mathrm{MPa}$ in the form of a Helmholtz function. A correction term additional to the ideal-gas part of IAPWS-95 below $130 \mathrm{~K}$ is proposed in Appendix $\mathrm{C}$ of this paper, extending its validity to $50 \mathrm{~K}$, for consistency with the latest formulas for the sublimation pressure and the sublimation enthalpy of ice Ih (Feistel and Wagner, 2007; IAPWS, 2008b).

An industrial humid-air description, intended for applications at elevated pressure and temperature, was recently proposed by Aleksandrov and Orlov (2007), based on the dryair equation of Lemmon et al. (2000) in conjunction with the IAPWS Industrial Formulation IF97 (Wagner and Kretzschmar, 2008) for fluid water and an earlier equation for ice (Feistel and Wagner, 2005), although without including virial coefficients for air-water interaction. The approach of Nelson and Sauer (Gatley, 2005) is similar. The "SKU" model of Herrmann et al. (2009c, 2010) also is an ideal-mixture combination of the real-gas formulation of Lemmon et al. (2000) with IAPWS (2009a) for water vapour (ideal mixtures are models which may consider non-ideal properties of the pure components but neglect any interaction between the particles of different chemical species). Herrmann et al. (2009c) provide an extensive comparison of different humid-air equations and experimental data.

Millero (2001, p. 291) provides a vapour pressure formula for seawater that uses the pure-water vapour pressure of Ambrose and Lawrenson (1972) with a pressure-independent three-term salinity correction. The related table for practical

\footnotetext{
${ }^{7}$ CIPM: International Committee for Weights and Measures, www.bipm.org/en/committees/cipm/.
} 
salinity $S_{\mathrm{P}}=35$ refers to Millero and Leung (1976), who provide a different, two-term salinity correction. The temperature scale can be assumed to be IPTS- $68^{8}$, the salinity scale is PSS- $78^{9}$. No uncertainty estimate is given. Kennish (2001, p. 62) provides a table with reference to Millero (1996), which probably means the same formula as given in Millero (1983) and Millero (2001). The earlier edition of the CRC Handbook of Marine Science (Walton Smith, 1974, p. 49) reported the vapour pressure lowering of seawater from Robinson (1954) and Arons and Kientzler (1954). The formula is pressure-independent. Siedler and Peters (1986, p. 252) refer to Millero (1983), who in turn provides the same formula as Millero (2001). Gill (1982) refers to empirical formulas of List (1951) and Kraus (1972) for the vapour pressure over water and seawater. Dietrich et al. (1975, p. 68) provide a graph for the vapour pressure lowering based on measurements of Higashi et al. (1931) and Arons and Kientzler (1954). No uncertainty estimates are given and the temperature and salinity scales are not specified. The formula is pressure-independent.

Linke and Baur (1970, p. 476) provide a standard meteorological formula that is attributed to G. Hofmann (without giving the reference, which probably is Hofmann, 1955, 1956) for pure water with a linear salinity correction from Defant $(1961$, p. 45). The latter in turn uses results from Witting (1908) without specifying that reference. No uncertainty estimates are given, the temperature and salinity scales are not reported, and the formula is pressure-independent. Tetens' (1930) formula for the saturated vapour pressure is also common in meteorology; a recent update was recommended by Bolton (1980). A review of vapour-pressure formulas over pure water and ice was given by Sonntag (1982) with explicit conversion to the IPTS-68 temperature scale; the effect of the conversion to ITS-90 on the saturation vapour pressure was analysed by Sonntag (1990). Saturated vapour pressure data and formulae since 1740 were reviewed by Sonntag (1998) who expressed his regret regarding the lack of internationally recognised meteorological standards for these properties. For very accurate vapour pressure formulas such as that of Weiss and Price (1980) for seawater, based on Robinson's (1954) careful experiments, the temperature conversion to the recent ITS-90 temperature scale is essential (Feistel, 2008a). An early review on seawater evaporation was published by Montgomery (1940). Formulas used for the latent heat in numerical ocean models are given by Smith (1988); simple expressions for the evaporation and sublimation heat as functions of temperature are available from Gill (1982).

Various properties of marine aerosols are reviewed by O'Dowd et al. (1997) and Seinfeld and Pandis (1998). The adjustment of the equilibrium droplet size distribution of sea

\footnotetext{
${ }^{8}$ IPTS-68: International Practical Temperature Scale of 1968 (Goldberg and Weir, 1992).

${ }^{9}$ PSS-78: Practical Salinity Scale 1978 (Unesco, 1981).
}

smoke by differential evaporation and condensation is controlled by the so-called Köhler equation (Köhler, 1936; Seinfeld and Pandis, 1998; Jacobson, 2005). This relaxation process is similar to the Ostwald ripening of nuclei that emerge along with phase transitions of the first kind (Ostwald, 1896; Schmelzer and Schweitzer, 1987), as e.g. encountered in cloud formation processes (Rogers and Yau, 1989; Pruppacher and Klett, 1997; Hellmuth, 2006). In this paper we do not consider the specific properties of aerosols or similar systems that are essentially influenced by surface-tension effects.

Consistent with the Releases of IAPWS (2008b, 2009a, 2009b), valid over the full range of ambient temperatures including conditions in the high atmosphere at high latitudes, the vapour pressure over ice (i.e., the sublimation pressure) was compiled by Feistel and Wagner (2007) and is available from an IAPWS Release (IAPWS, 2008b). The vapour pressure over sea ice (i.e. ice in equilibrium with brine pockets) and its relation to the brine salinity is discussed in Sect. 8 .

As is evident from this brief review, numerous different formulas are in practical use for the particular properties of water, seawater and air, on different temperature scales, with different accuracies and over different ranges of validity, with uncertain completeness or mutual consistency. Quantitative information is scarce about fundamental quantities such as entropy or enthalpy of humid air, or the latent heat of seawater. In this paper we propose to replace this inhomogeneous collection by consistent, thermodynamically rigorous formulas derived from a minimum set of comprehensive and most accurate thermodynamic potentials of fluid water, ice, seawater (Feistel, 2008a, b; Feistel et al., 2008b) and humid air which provide all thermodynamic equilibrium properties of those substances as well as their combinations and mutual phase equilibria over a wide range of conditions. When an official standard formulation for humid air becomes available which covers wider ranges of conditions than the present CIPM-2007 formula (Picard et al., 2008), the building-block concept proposed here will permit its easy incorporation into the suggested system of equations derived by thermodynamic rules.

Simple correlation functions for particular thermodynamic properties have advantages for certain purposes; they are easy to use and numerical implementations execute quickly. The comprehensive formulation proposed in this paper can provide error estimates and validity limits for such existing formulas. For high-speed applications, it can be used to derive from its computed data points tailored, very accurate and consistent new correlation equations. The time it takes to determine tailored formulae or to compute and store tabulated values in look-up tables is irrelevant for their later usage. The sea-air functions implemented in the library (Feistel et al., 2009; Wright et al., 2009) permit the computation of look-up tables for practically any desired combination of input and output properties, since the thermodynamic potentials provide a complete description. Real-time models 
require the highest computation speeds; so, we believe that our equations may well feed such models with the most accurate properties available. However, we recognize that for particular applications such as in oceanographic or climate models, computation (or look-up) speed may be critical, too.

\section{Helmholtz and Gibbs functions of fluid water, ice and seawater}

The new standard formulation for thermodynamic properties of seawater developed cooperatively by the SCOR/IAPSO WG 127 (Feistel, 2008a; IOC, 2010) and IAPWS (2008) consists of the saline part of the Gibbs function, $g^{\mathrm{S}}\left(S_{\mathrm{A}}, T, P\right)$ which describes the salinity correction to the specific Gibbs energy of pure liquid water that can be derived from the Helmholtz potential for fluid (i.e. liquid and gaseous) water, and a Gibbs function $g^{\mathrm{Ih}}(T, P)$ for ice (IAPWS, 2009a, 2009b; Feistel et al., 2008b). Mathematical details of those correlation functions are given in the appendix.

The Gibbs function of seawater, $g^{\mathrm{SW}}$,

$g^{\mathrm{SW}}\left(S_{\mathrm{A}}, T, P\right)=g^{\mathrm{W}}(T, P)+g^{\mathrm{S}}\left(S_{\mathrm{A}}, T, P\right)$,

as a function of absolute salinity $S_{\mathrm{A}}$, absolute temperature $T$ and absolute pressure $P$ refers to a Gibbs function of water, $g^{\mathrm{W}}$, as its zero-salinity limit, and a saline part, $g^{\mathrm{S}}$, which is explicitly available from IAPWS (2008). Rather than the Gibbs function $g^{\mathrm{W}}$, the IAPWS-95 standard provides a Helmholtz function for fluid water, $f^{\mathrm{F}}(T, \rho)$, depending on temperature and density. Below the critical temperature, the related equation for the pressure,

$P=\rho^{2}\left(\frac{\partial f^{\mathrm{F}}}{\partial \rho}\right)$

has two different inverse functions of practical relevance, one for the liquid density, $\rho^{\mathrm{W}}(T, P)$, and one for the vapour density, $\rho^{\mathrm{V}}(T, P)$, which can be computed iteratively from Eq. (3.2). With this liquid density, the Gibbs function of liquid water is obtained from

$g^{\mathrm{W}}(T, P)=f^{\mathrm{F}}\left(T, \rho^{\mathrm{W}}\right)+P / \rho^{\mathrm{W}}$,

and similarly for the Gibbs function of water vapour, $g^{\mathrm{V}}$.

Together with $g^{\mathrm{S}}$ available from the IAPWS Release 2008, Eq. (3.3) provides the full Gibbs function (3.1) of seawater, $g^{\mathrm{SW}}$, from which all thermodynamic properties of seawater are available by thermodynamic rules, as e.g. the entropy

$s^{\mathrm{SW}}\left(S_{\mathrm{A}}, T, P\right)=-\left(\frac{\partial g \mathrm{SW}}{\partial T}\right)_{S_{\mathrm{A}}, P}$

or the enthalpy,

$h^{\mathrm{SW}}\left(S_{\mathrm{A}}, T, P\right)=g^{\mathrm{SW}}+T s^{\mathrm{SW}}=g^{\mathrm{SW}}-T\left(\frac{\partial g^{\mathrm{SW}}}{\partial T}\right)_{S, P}$
In particular for the description of phase equilibria with seawater, partial specific properties are conveniently derived from the Gibbs function. If, for example, $H^{\mathrm{SW}}$ is the enthalpy of a sample of seawater that contains the masses $m^{\mathrm{W}}$ of water and $m^{\mathrm{S}}$ of salt, respectively, the partial specific enthalpy $H_{\mathrm{W}}^{\mathrm{SW}}$ of water in seawater is defined by (Glasstone, 1947)

$H_{\mathrm{W}}^{\mathrm{SW}}=\left(\frac{\partial H^{\mathrm{SW}}}{\partial m^{\mathrm{W}}}\right)_{m^{\mathrm{s}}, T, P}$.

Transformation to the absolute salinity, $S_{\mathrm{A}}=m^{\mathrm{S}} /\left(m^{\mathrm{W}}+m^{\mathrm{S}}\right)$, and the specific enthalpy, $h^{\mathrm{SW}}=H^{\mathrm{SW}} /\left(m^{\mathrm{W}}+m^{\mathrm{S}}\right)$, yields for the partial specific enthalpy the expression

$$
\begin{aligned}
H_{\mathrm{W}}^{\mathrm{SW}} & =h^{\mathrm{SW}}+\left(m^{\mathrm{W}}+m^{\mathrm{S}}\right)\left(\frac{\partial h^{\mathrm{SW}}}{\partial S_{\mathrm{A}}}\right)_{T, P}\left(\frac{\partial S_{\mathrm{A}}}{\partial m^{\mathrm{W}}}\right)_{m^{\mathrm{S}}} \\
& =h^{\mathrm{SW}}-S_{\mathrm{A}}\left(\frac{\partial h^{\mathrm{SW}}}{\partial S_{\mathrm{A}}}\right)_{T, P} .
\end{aligned}
$$

By means of Eqs. (3.5) and (3.7), $H_{\mathrm{W}}^{\mathrm{SW}}$ is computed from the Gibbs function of seawater. Since $H_{\mathrm{W}}^{\mathrm{SW}}$ depends on one freely adjustable constant (see Appendix E), the absolute energy of water, only differences between partial specific enthalpies rather than their absolute values can be measured in thermodynamic experiments. With respect to different phases that contain water, an important example for such a difference is the latent heat of the related phase transition.

If the Gibbs energy $G^{\mathrm{SW}}$ of seawater is used in Eq. (3.6) rather than $H^{\mathrm{SW}}$, then the related result (3.7) is the partial specific Gibbs energy of water in seawater which equals the chemical potential of water in seawater (Landau and Lifschitz, 1987; IOC, 2010).

The freely adjustable constants representing the absolute energy and entropy of water and sea salt are specified by the reference state conditions of vanishing internal energy and entropy of liquid water at the triple point and zero entropy and enthalpy of seawater at the standard ocean state (Feistel et al., 2008b). The related terms of the Gibbs functions which are determined by these conditions are independent of pressure and of order 0 and 1 in temperature and salinity, respectively (Fofonoff, 1962; Feistel and Hagen, 1995).

\section{Helmholtz function for dry air}

Air is a mixture of gases, Table A2, as described recently by Picard et al. (2008), with only minor variability of its composition under ambient conditions. A Helmholtz function for dry air was published by Lemmon et al. (2000) in the form of the molar Helmholtz energy, $f^{\mathrm{A}, \mathrm{mol}}\left(T, \rho^{\mathrm{mol}}\right)$, depending on absolute temperature $T$ (ITS-90) and molar air density, $\rho^{\mathrm{mol}}$. For consistency with the description of water and seawater, we will use in this paper the specific (rather than the molar) 
Helmholtz energy, $f^{\mathrm{A}}$, as the thermodynamic Helmholtz potential,

$$
f^{\mathrm{A}}\left(T, \rho^{\mathrm{A}}\right)=\frac{F^{\mathrm{A}}}{m^{\mathrm{A}}}=\frac{1}{M^{\mathrm{A}, \mathrm{L}}} f^{\mathrm{A}, \mathrm{mol}}\left(T, \frac{\rho^{\mathrm{A}}}{M^{\mathrm{A}, \mathrm{L}}}\right),
$$

which depends on the mass density of dry air, $\rho^{\mathrm{A}}=m^{\mathrm{A}} / V$. For consistency we use in the conversion (4.1) the original estimate of the molar mass of dry air given by Lemmon et al. (2000), $M^{\mathrm{A}, \mathrm{L}}=28.9586(2) \mathrm{g} \mathrm{mol}^{-1}$, rather than the latest estimate by Picard et al. (2008), $M^{\mathrm{A}}=28.96546 \mathrm{~g} \mathrm{~mol}^{-1}$ (Table A2). In Eq. (4.1), $m^{\mathrm{A}}$ is the mass of air contained in the volume $V$, and $F^{\mathrm{A}}$ is the (extensive) Helmholtz energy of this air sample.

The Helmholtz function (4.1) for dry air adopted from Lemmon et al. (2000) is defined in Eq. (B1) in the Appendix. The absolute energy and the absolute entropy of dry air is specified here by the reference-state condition of vanishing entropy and enthalpy at the standard ocean state, $T_{0}=273.15 \mathrm{~K}, P_{0}=101325 \mathrm{~Pa}$. The related freely adjustable terms of $f^{\mathrm{A}}\left(T, \rho^{\mathrm{A}}\right)$ are independent of $\rho^{\mathrm{A}}$ and multiply powers 0 and 1 in $T$.

Various thermophysical properties of dry air are available from the formulas given in the following section for the limiting case of vanishing humidity.

\section{Helmholtz and Gibbs functions for humid air}

The Helmholtz function $f^{\mathrm{V}}\left(T, \rho^{\mathrm{V}}\right)$ of water vapour is available from the IAPWS-95 formulation, Sect. 3 and Appendix $\mathrm{C}$, and that of dry air, $f^{\mathrm{A}}\left(T, \rho^{\mathrm{A}}\right)$, from Appendix B and Eq. (4.1) above. If a mass $m^{\mathrm{V}}$ of vapour is mixed with a mass $m^{\mathrm{A}}$ of dry air in a volume $V$, the virial expansion of the Helmholtz energy $F^{\mathrm{AV}}$ of the humid-air system up to three-particle vapour-air interactions reads (Appendix D),

$$
\begin{aligned}
& F^{\mathrm{AV}}\left(m^{\mathrm{V}}, m^{\mathrm{A}}, T, V\right)=m^{\mathrm{V}} f^{\mathrm{V}}\left(T, \frac{m^{\mathrm{V}}}{V}\right)+m^{\mathrm{A}} f^{\mathrm{A}}\left(T, \frac{m^{\mathrm{A}}}{V}\right) \\
& +2 \frac{R T}{V} \frac{m^{\mathrm{A}} m^{\mathrm{V}}}{M^{\mathrm{A}} M^{\mathrm{W}}}\left\{B_{\mathrm{AW}}(T)+\frac{3}{4 V}\left[\frac{m^{\mathrm{A}}}{M^{\mathrm{A}}} C_{\mathrm{AAW}}(T)+\frac{m^{\mathrm{V}}}{M^{\mathrm{W}}} C_{\mathrm{AWW}}(T)\right]\right\}
\end{aligned}
$$

In Eq. (5.1), $R$ is the molar gas constant, and $M^{\mathrm{A}}, M^{\mathrm{W}}$ are the molar masses of air and water, respectively. The second molar virial coefficient $B_{\mathrm{AW}}(T)$ is available from Harvey and Huang (2007), and the third molar virial coefficients $C_{\mathrm{AAW}}(T)$ and $C_{\mathrm{AWW}}(T)$ of air-vapour interaction are reported by Hyland and Wexler (1983b). We note that the Helmholtz functions $f^{\mathrm{V}}$ and $f^{\mathrm{A}}$ that we have chosen to use in Eq. (5.1) are complete expressions rather than expansions in terms of powers of density; consequently, they include contributions corresponding to higher powers of density than included in the cross-virial terms represented by the third term, see Appendix D. Eq. (5.1) is thus an inhomogeneous approximation formula with respect to the powers of density and the related correlation clusters. This approach provides accurate results for higher densities if $m^{\mathrm{A}} \ll m^{\mathrm{V}}$ or $m^{\mathrm{V}} \ll m^{\mathrm{A}}$ where the contributions from one component are strongly dominating and contributions from 4th and higher order cross-virial terms remain negligible. The absence of these higher cross-virial coefficients will not relevantly affect the accuracy of Eq. (5.1) for meteorological applications because of the smallness of non-ideal effects, Fig. 1, under natural atmospheric conditions of temperature, pressure and humidity.

Defining the mass of humid air by $m^{\mathrm{AV}}=m^{\mathrm{A}}+m^{\mathrm{V}}$ and the mass fraction of dry air by $A=m^{\mathrm{A}} / \mathrm{m}^{\mathrm{AV}}$, we find for the Helmholtz function of humid air the expression

$$
\begin{aligned}
& f^{\mathrm{AV}}\left(A, T, \rho^{\mathrm{AV}}\right)=\frac{F^{\mathrm{AV}}}{m^{\mathrm{AV}}}=(1-A) f^{\mathrm{V}}\left(T,(1-A) \rho^{\mathrm{AV}}\right)(5.2) \\
& +A f^{\mathrm{A}}\left(T, A \rho^{\mathrm{AV}}\right)+2 \rho^{\mathrm{AV}} \frac{A(1-A) R T}{M^{\mathrm{A}} M^{\mathrm{W}}} \\
& \left\{B_{\mathrm{AW}}(T)+\frac{3}{4} \rho^{\mathrm{AV}}\left[\frac{A}{M^{\mathrm{A}}} C_{\mathrm{AAW}}(T)+\frac{(1-A)}{M^{\mathrm{W}}} C_{\mathrm{AWW}}(T)\right]\right\}
\end{aligned}
$$

Here, $\rho^{\mathrm{AV}}=m^{\mathrm{AV}} / V$ is the density of humid air, $q=1-A=$ $m^{\mathrm{V}} / m^{\mathrm{AV}}$ is its specific humidity, $(1-A) \rho^{\mathrm{AV}}=m^{\mathrm{V}} / V$ is its absolute humidity, and $r=(1-A) / A=m^{\mathrm{V}} / \mathrm{m}^{\mathrm{A}}$ is the humidity ratio or mixing ratio (van Wylen and Sonntag, 1965; Gill, 1982; Emanuel, 1994; Pruppacher and Klett, 1997). The relation to relative humidity is given in Sects. 7 and 10, and Fig. 14 therein.

Later in this text, we will frequently omit for simplicity of the formulae the superscript AV at the density of humid air, $\rho^{\mathrm{AV}} \equiv \rho$, if there is no risk of confusion.

The use of $A$ rather than $q$ as the composition variable of humid air is not common. This somewhat unfamiliar decision was made independently of the typical ambient mass ratios $(q \ll 1)$. The formalisms used here are very similar for the ocean and the atmosphere. Water in its three phases forms the reference system; in particular its two fluid phases are described by one and the same Helmholtz potential (IAPWS95) for rigorous consistency between ocean and atmosphere. Thus, as in nature, also in this theoretical model the water is exactly "the same" on both sides of the sea surface. Added to these fluid phases of water are natural mixtures of almost constant composition, sea salt in the liquid and dry air in the gas phase. For these additives, their pure properties are described and corrected by a density expansion with respect to their interaction with water. As a result, the equations for humid air look very similar to those of seawater if just the mass fraction $A$ of the one additive is exchanged with the other, $S_{\mathrm{A}}$.

The Helmholtz potential (5.2) is formally a symmetric function in the fractions of air and of vapour. Its validity is not restricted to small specific humidity, $q=(1-A)$, such as some 1-3\% often assumed for empirical equations used in meteorology; see for example Figs. 3 and 13 in the following sections. Equation (5.2) can therefore be applied even to physical situations in which dry air is the minor fraction, 

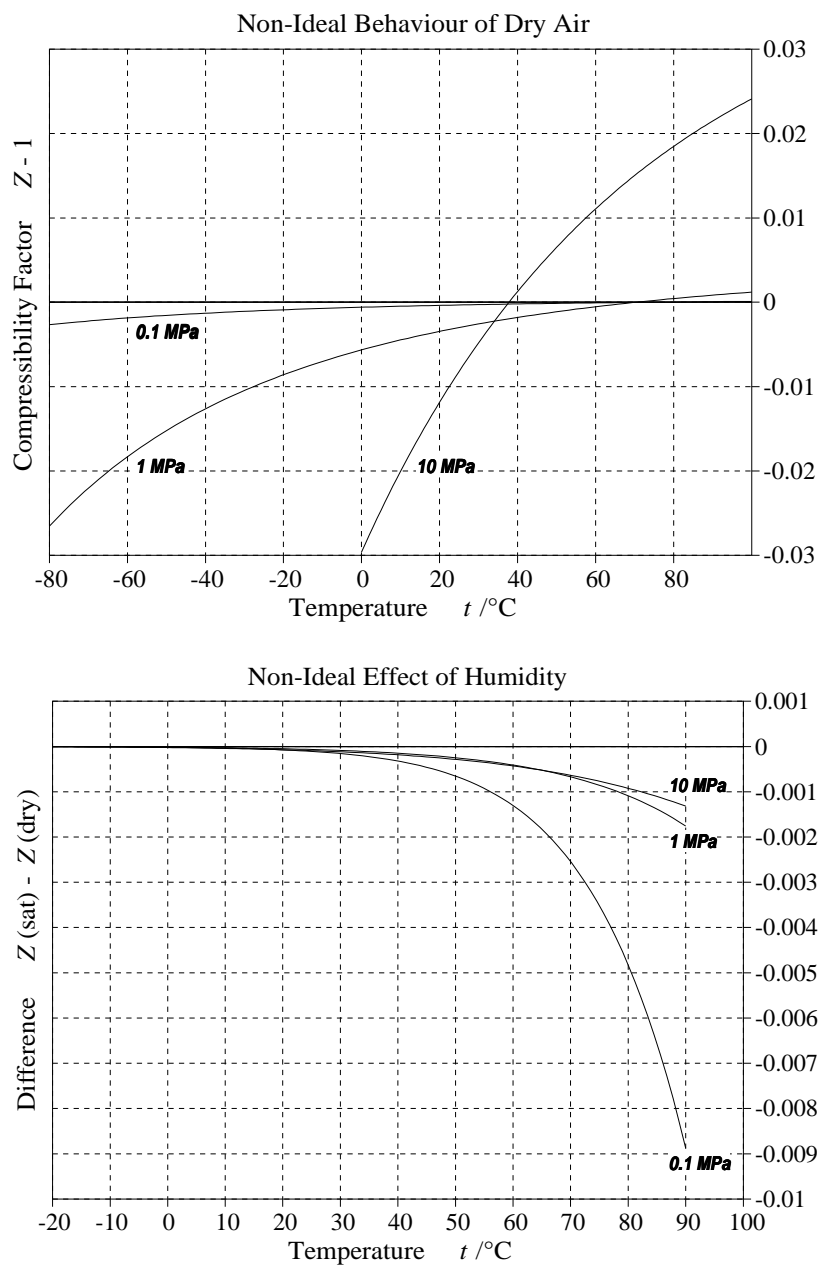

Fig. 1. Non-ideal behaviour $\left(Z^{\mathrm{AV}}-1\right)$, Eq. (5.3), of dry (upper panel) and saturated (lower panel) air for several values of $T$ and $P$.

such as condensers of desalination plants or headspaces over subglacial lakes. The most relevant restriction for the accuracy of Eq. (5.2) is the truncation error of the virial expansion, i.e., its omitted subsequent terms proportional to $A^{3}(1-A) \rho^{3}, A^{2}(1-A)^{2} \rho^{3}$ and $A(1-A)^{3} \rho^{3}$ must be negligibly small. Hyland and Wexler (1983b) require pressures less than $5 \mathrm{MPa}$ for saturated humid air; this condition implies limitations to the air density in dependence of the air fraction. The comparison with experimental data shown in the appendix, Fig. D1, suggests that the current formulation of Eq. (5.2) can safely be used up to densities of $100 \mathrm{~kg} \mathrm{~m}^{-3}$ of saturated humid air. Results remain reasonable beyond this point, but the uncertain third and the missing fourth and higher cross-virial coefficients are clearly relevant beyond $200 \mathrm{~kg} \mathrm{~m}^{-3}$.

Describing the deviation of the equation of state from ideal-gas behaviour, the compressibility factor $Z_{\mathrm{AV}}$ of humid air is defined as (van Wylen and Sonntag, 1965),
$Z_{\mathrm{AV}}=\frac{P}{\rho^{\mathrm{AV}} R_{\mathrm{AV}} T}=\frac{\rho^{\mathrm{AV}}}{R_{\mathrm{AV}} T}\left(\frac{\partial f^{\mathrm{AV}}}{\partial \rho^{\mathrm{AV}}}\right)_{A, T}$,

where $R_{\mathrm{AV}}$ is the (effective) specific gas "constant" of humid air, as a function of the air fraction $A$,

$R_{\mathrm{AV}}=\frac{R}{M^{\mathrm{AV}}}=R\left[\frac{1-A}{M^{\mathrm{W}}}+\frac{A}{M^{\mathrm{A}}}\right]=(1-A) R_{\mathrm{W}}+A R_{\mathrm{A}}$

and $M^{\mathrm{AV}}$ its mean molar mass,

$M^{\mathrm{AV}}=x_{\mathrm{V}}^{\mathrm{AV}} M^{\mathrm{W}}+\left(1-x_{\mathrm{V}}^{\mathrm{AV}}\right) M^{\mathrm{A}}=\left[\frac{1-A}{M^{\mathrm{W}}}+\frac{A}{M^{\mathrm{A}}}\right]^{-1}$,

where the mole fraction $x_{\mathrm{V}}^{\mathrm{AV}}$ of vapour is given by

$x_{\mathrm{V}}^{\mathrm{AV}}=\frac{(1-A) / M^{\mathrm{W}}}{(1-A) / M^{\mathrm{W}}+A / M^{\mathrm{A}}}=\frac{r}{r+M^{\mathrm{W}} / M^{\mathrm{A}}}$.

The deviation of the compressibility factor $Z_{\mathrm{AV}}$, as defined in Eq. (5.3), from unity is shown in Fig. 1 for dry and saturated air for several values of $T$ and $P$. Selected values for $Z_{\mathrm{AV}}$ are reported by Pruppacher and Klett (1997). The compressibility factor $Z$ of fluids is generally small in the vicinity of the critical point; the critical value is $Z_{\mathrm{c}}=0.375$ for the vander-Waals gas, 0.29 for noble gases (Guggenheim, 1967) and 0.23 for water (Stanley, 1971). In statistical thermodynamics, the compressibility factor is the logarithm of the grand canonical partition function and therefore a thermodynamic potential in terms of temperature, density and chemical potential (Landau and Lifschitz, 1987) which is regarded as the Landau free energy or the Landau potential (Goodstein, 1975) divided by $R T$.

For the total pressure of humid air we get from Eq. (5.2), compare Eq. (D11),

$$
\begin{aligned}
P & =-\left(\frac{\partial F^{\mathrm{AV}}}{\partial V}\right)_{m^{\mathrm{A}}, m^{\mathrm{V}}, T} \\
& =\left(\rho^{\mathrm{AV}}\right)^{2}\left(\frac{\partial f^{\mathrm{AV}}}{\partial \rho^{\mathrm{AV}}}\right)_{A, T}=\left(\rho^{\mathrm{AV}}\right)^{2}\left[(1-A)^{2} f_{\rho^{\mathrm{V}}}^{\mathrm{V}}+A^{2} f_{\rho^{\mathrm{A}}}^{\mathrm{A}}\right] \\
& +A(1-A)\left(\rho^{\mathrm{AV}}\right)^{2} \frac{R T}{M^{\mathrm{A}} M^{\mathrm{W}}} \\
& \left\{2 B_{\mathrm{AW}}(T)+3 \rho^{\mathrm{AV}}\left[\frac{A}{M^{\mathrm{A}}} C_{\mathrm{AAW}}(T)+\frac{(1-A)}{M^{\mathrm{W}}} C_{\mathrm{AWW}}(T)\right]\right\}
\end{aligned}
$$

from which the partial pressure of vapour, $P^{\mathrm{V}}$, is obtained by multiplication with $x_{\mathrm{V}}^{\text {AV }}$ (Guggenheim, 1967). The subscripts on $f_{\rho^{\mathrm{V}}}^{\mathrm{V}}$ and $f_{\rho^{\mathrm{A}}}^{\mathrm{A}}$ are meant as partial derivatives with respect to the particular density arguments of those functions, rather than derivatives with respect to the total density of humid air, $\rho^{\mathrm{AV}}$, in Eq. (5.7). 
Computed from Eqs. (5.7) or (5.3), the ratio

$$
\begin{aligned}
T_{\mathrm{V}} & =\frac{P M^{\mathrm{A}}}{R \rho^{\mathrm{AV}}}=T Z_{\mathrm{AV}} \frac{A M^{\mathrm{W}}+(1-A) M^{\mathrm{A}}}{M^{\mathrm{W}}} \\
& =T Z_{\mathrm{AV}} \frac{1+r M^{\mathrm{A}} / M^{\mathrm{W}}}{1+r}
\end{aligned}
$$

is known in meteorology as the virtual temperature of humid air (Rogers and Yau, 1989; Jacobson, 2005; Hantel and Mayer, 2006).

The specific entropy of humid air follows from

$$
s^{\mathrm{AV}}=-\frac{1}{m^{\mathrm{AV}}}\left(\frac{\partial F^{\mathrm{AV}}}{\partial T}\right)_{m^{\mathrm{A}}, m^{\mathrm{V}}, V}=-\left(\frac{\partial f^{\mathrm{AV}}}{\partial T}\right)_{A, V}
$$

as

$$
\begin{aligned}
s^{\mathrm{AV}} & =-(1-A) f_{T}^{\mathrm{V}}-A f_{T}^{\mathrm{A}}-2 A(1-A) \rho^{\mathrm{AV}} \frac{R T}{M^{\mathrm{A}} M^{\mathrm{W}}} \\
& \times\left\{\frac{B_{\mathrm{AW}}}{T}+\frac{\mathrm{d} B_{\mathrm{AW}}}{\mathrm{d} T}+\frac{3}{4} \rho^{\mathrm{AV}}\left[\frac{A}{M^{\mathrm{A}}}\left(\frac{C_{\mathrm{AAW}}}{T}+\frac{\mathrm{d} C_{\mathrm{AAW}}}{\mathrm{d} T}\right)\right.\right. \\
& \left.\left.+\frac{(1-A)}{M^{\mathrm{W}}}\left(\frac{C_{\mathrm{AWW}}}{T}+\frac{\mathrm{d} C_{\mathrm{AWW}}}{\mathrm{d} T}\right)\right]\right\}
\end{aligned}
$$

and the specific enthalpy from Eqs. (3.3), (3.5) as

$h^{\mathrm{AV}}=f^{\mathrm{AV}}+P / \rho^{\mathrm{AV}}+T s^{\mathrm{AV}}$.

Here, the functions $f^{\mathrm{AV}}, P$ and $s^{\mathrm{AV}}$ are available from Eqs. (5.2), (5.7) and (5.10), respectively.

Equation (5.10) is consistent with the air-vapour interaction terms of the virial formula for the entropy given by Hyland and Wexler (1983b, their Eqs. 2, 3 and 7).

The Gibbs function of humid air, $g^{\mathrm{AV}}$, follows from its Helmholtz function (5.2) via the Legendre transform

$g^{\mathrm{AV}}=f^{\mathrm{AV}}+\rho^{\mathrm{AV}}\left(\frac{\partial f^{\mathrm{AV}}}{\partial \rho^{\mathrm{AV}}}\right)_{A, T}$.

The function $g^{\mathrm{AV}}$ can be written in terms of the chemical potentials of air in humid air, $\mu^{\mathrm{A}}$, and of vapour in humid air, $\mu^{\mathrm{V}}$, as (Gibbs-Duhem equation, Landau and Lifschitz, 1987; Feistel and Marion, 2007; Feistel, 2008a; IOC, 2010)

$g^{\mathrm{AV}}=A \mu^{\mathrm{A}}+(1-A) \mu^{\mathrm{V}}$

which obey the relations

$\mu^{\mathrm{A}}=g^{\mathrm{AV}}+(1-A)\left(\frac{\partial g^{\mathrm{AV}}}{\partial A}\right)_{T, P}$

and

$\mu^{\mathrm{V}}=g^{\mathrm{AV}}-A\left(\frac{\partial g^{\mathrm{AV}}}{\partial A}\right)_{T, P}$.

From the Gibbs function (5.12), the entropy (5.9) is computed as

$s^{\mathrm{AV}}(A, T, P)=-\left(\frac{\partial g^{\mathrm{AV}}}{\partial T}\right)_{A, P}$,

and the enthalpy in the form,
$h^{\mathrm{AV}}(A, T, P)=g^{\mathrm{AV}}+T s^{\mathrm{AV}}=g^{\mathrm{AV}}-T\left(\frac{\partial g^{\mathrm{AV}}}{\partial T}\right)_{A, P}$.

The specific isobaric heat capacity is $c_{P}^{\mathrm{AV}}=$ $\left(\partial h^{\mathrm{AV}} / \partial T\right)_{A, P}=T\left(\partial s^{\mathrm{AV}} / \partial T\right)_{A, P}$.

In particular for the description of phase equilibria with humid air, partial specific properties are conveniently derived from the Gibbs function. If, for example, $H^{\mathrm{AV}}$ is the enthalpy of a sample of humid air that contains the masses $m^{\mathrm{V}}$ of vapour and $m^{\mathrm{A}}$ of air, respectively, the partial specific enthalpy $H_{\mathrm{V}}^{\mathrm{AV}}$ of water in humid air is defined by (Glasstone, 1947)

$H_{\mathrm{V}}^{\mathrm{AV}}=\left(\frac{\partial H^{\mathrm{AV}}}{\partial m^{\mathrm{V}}}\right)_{m^{\mathrm{A}}, T, P}$.

Transformation to the air fraction, $A=m^{\mathrm{A}} /\left(m^{\mathrm{V}}+m^{\mathrm{A}}\right)$, and the specific enthalpy, $h^{\mathrm{AV}}=H^{\mathrm{AV}} /\left(m^{\mathrm{V}}+m^{\mathrm{A}}\right)$, yields for the partial specific enthalpy the expression

$$
\begin{aligned}
H_{\mathrm{V}}^{\mathrm{AV}} & =h^{\mathrm{AV}}+\left(m^{\mathrm{V}}+m^{\mathrm{A}}\right)\left(\frac{\partial h^{\mathrm{AV}}}{\partial A}\right)_{T, P}\left(\frac{\partial A}{\partial m^{\mathrm{V}}}\right)_{m^{\mathrm{A}}} \\
& =h^{\mathrm{AV}}-A\left(\frac{\partial h^{\mathrm{AV}}}{\partial A}\right)_{T, P} .
\end{aligned}
$$

By means of Eqs. (5.17) and (5.19), $H_{\mathrm{V}}^{\mathrm{AV}}$ is computed from the Gibbs function of humid air.

Exploiting the entropy (5.16), the (absolute) potential temperature $\theta^{\mathrm{AV}}(A, T, P)$ (in $\mathrm{K}$ ) of humid air referenced to standard pressure is obtained by solving (numerically) the equation

$s^{\mathrm{AV}}\left(A, \theta^{\mathrm{AV}}, P_{0}\right)=s^{\mathrm{AV}}(A, T, P)$

where $P_{0}=101325 \mathrm{~Pa}$. Equivalently, the potential temperature follows from the entropy derivative of the enthalpy (5.11) of humid air (Feistel and Hagen, 1995; Feistel, 2008), as

$\theta^{\mathrm{AV}}=\left(\frac{\partial h^{\mathrm{AV}}}{\partial s}\right)_{A, P=P_{0}}$,

where the right side is evaluated at the reference pressure, $P=P_{0}$, and in-situ entropy.

The equivalent potential temperature $\theta_{\mathrm{e}}^{\mathrm{AV}}$ is the potential temperature that a parcel of humid air would have if all its vapour were condensed and the latent heat released used to heat the parcel (Jacobson, 2005). If a mass fraction $(1-A)$ of liquid water with the initial temperature $\theta_{\mathrm{e}}^{\mathrm{AV}}$ is irreversibly evaporated into dry air with the mass fraction $A$ at the surface pressure $P_{0}$, the resulting humid air is cooled by this process to the temperature $\theta^{\mathrm{AV}}$ determined from,

$(1-A) h^{\mathrm{W}}\left(\theta_{\mathrm{e}}^{\mathrm{AV}}, P_{0}\right)+A h^{\mathrm{A}}\left(\theta_{\mathrm{e}}^{\mathrm{AV}}, P_{0}\right)=h^{\mathrm{AV}}\left(A, \theta^{\mathrm{AV}}, P_{0}\right)$, 
because the enthalpy is conserved during an irreversible isobaric and adiabatic process rather than the entropy (Landau and Lifschitz, 1987; IOC, 2010). This equation implicitly provides the equivalent potential temperature $\theta_{\mathrm{e}}^{\mathrm{AV}}\left(A, \theta^{\mathrm{AV}}\right)$ as a function of the air fraction and the potential temperature of the original humid-air sample.

From the Gibbs function (5.12), the specific volume $v^{\mathrm{AV}}$ and the density $\rho^{\mathrm{AV}}$ of humid air at given $T$ and $P$ are computed as

$$
v^{\mathrm{AV}}(A, T, P)=\frac{1}{\rho^{\mathrm{AV}}}=\left(\frac{\partial g^{\mathrm{AV}}}{\partial P}\right)_{A, T} .
$$

The parcel's volume expansion during adiabatic uplift is obtained from the adiabatic compressibility,

$$
\kappa_{s}^{\mathrm{AV}}=-\frac{1}{v^{\mathrm{AV}}}\left(\frac{\partial v^{\mathrm{AV}}}{\partial P}\right)_{A, s}=-\frac{\left(\partial^{2} h^{\mathrm{AV}} / \partial P^{2}\right)_{A, T}}{\left(\partial h^{\mathrm{AV}} / \partial P\right)_{A, T}}=\frac{1}{\rho^{\mathrm{AV}} c^{2}} .
$$

Here, $c=\sqrt{(\partial P / \partial \rho)_{A, s}}$ is the sound speed.

The adiabatic lapse rate of humid air (recall that in some literature this is referred to as the "dry" adiabatic lapse rate rather than the "moist" adiabatic lapse rate) is computed from the enthalpy (5.11) or the Gibbs function (5.12) as

$$
\Gamma^{\mathrm{AV}}=\left(\frac{\partial T}{\partial P}\right)_{A, s}=\left(\frac{\partial^{2} h^{\mathrm{AV}}}{\partial s \partial P}\right)_{A}=-\frac{\left(\partial^{2} g^{\mathrm{AV}} / \partial T \partial P\right)_{A}}{\left(\partial^{2} g^{\mathrm{AV}} / \partial T^{2}\right)_{A, P}}
$$

or, by means of Table G2, from the Helmholtz function (5.2) at the given humid-air density, $\rho=\rho^{\mathrm{AV}}$, as

$$
\Gamma^{\mathrm{AV}}=\frac{f_{T \rho}^{\mathrm{AV}}}{\rho^{2}\left(f_{T \rho}^{\mathrm{AV}}\right)^{2}-\rho f_{T T}^{\mathrm{AV}}\left(2 f_{\rho}^{\mathrm{AV}}+\rho f_{\rho \rho}^{\mathrm{AV}}\right)} .
$$

Here, the subscripts of $f^{\mathrm{AV}}$ indicate partial derivatives at fixed $A$. The deviation between the lapse rates of dry air $(A=1)$ and of humid air $(A<1)$ in the atmosphere is usually less than 3\% (Seinfeld and Pandis, 1998), in contrast to the wet-air lapse rate, Eq. (7.19). Selected values of Eq. (5.26) computed at saturation humidity are displayed in Fig. 2.

The adiabatic virtual-temperature lapse rate (Jacobson, 2005) follows from the pressure derivative of the virtual temperature, Eq. (5.8), as

$$
\begin{aligned}
\Gamma_{\mathrm{V}}^{\mathrm{AV}} & =\left(\frac{\partial T_{\mathrm{V}}}{\partial P}\right)_{A, s}=\frac{M^{\mathrm{A}}}{R}\left(\frac{\partial\left(P v^{\mathrm{AV}}\right)}{\partial P}\right)_{A, s} \\
& =T_{\mathrm{V}}\left(\frac{1}{P}-\kappa_{s}^{\mathrm{AV}}\right)=T_{\mathrm{V}}\left(\frac{1}{P}-\frac{1}{\rho^{\mathrm{AV}} c^{2}}\right)
\end{aligned}
$$

Adiabatic compressibility and sound speed are given in Eq. (5.24).

In addition to the compressibility factor, Eq. (5.3), another common measure for the non-ideal behaviour of gases or gas mixtures is the fugacity (Glasstone, 1947; van Wylen and Sonntag, 1965; Guggenheim, 1967; IUPAC, 1997). It is defined in terms of chemical potentials in such a way that it coincides with the (partial) pressure of the gas in the idealgas limit. The fugacity $f_{\mathrm{AV}}$ of humid air is

$f_{\mathrm{AV}}(A, T, P)=P \exp \left(\frac{g^{\mathrm{AV}}-g^{\mathrm{AV}, \mathrm{id}}}{R_{\mathrm{AV}} T}\right)$

Here we have used the subscript AV on $f$ to distinguish the fugacity $f_{\mathrm{AV}}$ from the Helmholtz function $f^{\mathrm{AV}}$ of humid air. The pressure $P$ is obtained from Eq. (5.7), the ideal-gas limit $g^{\mathrm{AV}, \text { id }}$ is given in Eq. (H5).

The fugacity is related to the compressibility factor, $Z_{\mathrm{AV}}$, Eq. (5.3), by the differential equation (Glasstone, 1947; van Wylen and Sonntag, 1965),

$$
\frac{P}{f_{\mathrm{AV}}}\left(\frac{\partial f_{\mathrm{AV}}}{\partial P}\right)_{A, T}=Z_{\mathrm{AV}}=\frac{P}{\rho R_{\mathrm{AV}} T}
$$

Because $g^{\mathrm{AV}}=f^{\mathrm{AV}}+Z_{\mathrm{AV}} R_{\mathrm{AV}} T$, using Eqs. (5.3) and (5.12), the fugacity (5.28) can be expressed in terms of Helmholtz functions as

$f_{\mathrm{AV}}(A, T, P)=P \exp \left(\frac{f^{\mathrm{AV}}-f^{\mathrm{AV}, \mathrm{id}}}{R_{\mathrm{AV}} T}+Z_{\mathrm{AV}}-1\right)$.

The fugacity of vapour in humid air is

$f_{\mathrm{V}}(A, T, P)=x_{\mathrm{V}}^{\mathrm{AV}} P \exp \left(\frac{\mu^{\mathrm{V}}-\mu^{\mathrm{V}, \mathrm{id}}}{R_{\mathrm{W}} T}\right)$.

Here, $R_{\mathrm{W}}=R / M^{\mathrm{W}}$ is the specific gas constant of water, $\mu^{\mathrm{V}}$ is given in Eq. (5.15) and its ideal-gas limit in Eq. (H13).

The saturation of air with vapour is defined by the equilibrium between humid air and liquid water and will be considered in Sect. 7, i.e. as the limiting case for zero salinity of the sea-air properties derived in the next section.

\section{Gibbs function for sea air}

The Gibbs function $g$ SW

$g^{\mathrm{SW}}\left(S_{\mathrm{A}}, T, P\right)=g^{\mathrm{W}}(T, P)+g^{\mathrm{S}}\left(S_{\mathrm{A}}, T, P\right)$

of seawater (IAPWS, 2008a; Feistel, 2008a; Feistel et al., 2009; Wright et al., 2009) consist of a pure-water part, $g^{\mathrm{W}}$, available from the Helmholtz function $f^{\mathrm{W}}(T, \rho)$ given by the IAPWS-95 formulation, and a saline addition, $g^{\mathrm{S}}$, accounting for the solute regarded as sea salt. Here, $S_{\mathrm{A}}=$ $m^{\mathrm{S}} /\left(m^{\mathrm{W}}+m^{\mathrm{S}}\right)$ is the absolute salinity (Millero et al., 2008), expressed by the mass, $m^{\mathrm{S}}$, of salt dissolved in a mass of liquid water, $m^{\mathrm{W}}$.

In the composite system "sea air" consisting of seawater and humid air, these two phases are spatially separate and the mutual molecular interaction (surface effects) of their particles will be neglected. We may imagine a portion of the surface mixed layer of the ocean in contact with the atmospheric 


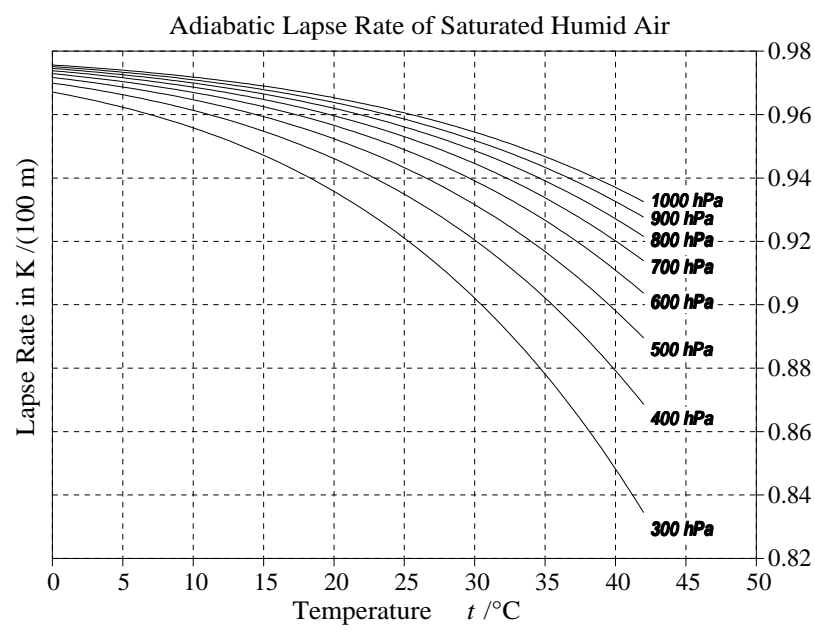

Fig. 2. Adiabatic lapse rate (5.26) of saturated humid air at different temperatures and pressures as indicated by the curves. Note that this lapse rate is termed "dry" in some literature. For convenience, the rate is shown per $100 \mathrm{~m}$ altitude difference as common in meteorology, rather than per pressure difference. For comparison, the lapse rate of wet air is shown in Fig. 7.

surface boundary layer, or droplets of sea spray floating in air, in thermodynamic equilibrium at their interface. As a simple example, a box containing certain masses of water, air and salt (in any initial form) will adjust to its equilibrium state in the form of seawater and humid air after some relaxation time. When the volume or the temperature of the box is changing, the related shifted liquid-gas equilibria can be computed from a Gibbs function of this heterogeneous system since each component has the same pressure and temperature. Thus, the Gibbs function $g^{\text {SA }}$ of sea air consists of the sum of a seawater part with the mass $m^{\mathrm{SW}}=m^{\mathrm{S}}+m^{\mathrm{W}}$, and a humid-air part with the mass $m^{\mathrm{AV}}=m^{\mathrm{A}}+m^{\mathrm{V}}$, forming the total sea-air mass $m^{\mathrm{SA}}=m^{\mathrm{SW}}+m^{\mathrm{AV}}$ :

$$
\begin{gathered}
g^{\mathrm{SA}}\left(w^{\mathrm{A}}, w^{\mathrm{S}}, T, P\right)=w^{\mathrm{AV}} g^{\mathrm{AV}}(A, T, P) \\
\quad+w^{\mathrm{SW}} g^{\mathrm{SW}}\left(S_{\mathrm{A}}, T, P\right)
\end{gathered}
$$

Here, the independent composition variables $w^{\mathrm{A}}=m^{\mathrm{A}} / m^{\mathrm{SA}}$ and $w^{\mathrm{S}}=m^{\mathrm{S}} / m^{\mathrm{SA}}$ are the mass fractions of air and of sea salt in sea air, respectively. The liquid mass fraction of the sea-air composite is $w^{\mathrm{SW}}=m^{\mathrm{SW}} / m^{\mathrm{SA}}=w^{\mathrm{S}} / S_{\mathrm{A}}$, the gaseous one is $w^{\mathrm{AV}}=m^{\mathrm{AV}} / m^{\mathrm{SA}}=w^{\mathrm{A}} / A$. Thus, from the mass conservation we infer the identity

$w^{\mathrm{A}} / A+w^{\mathrm{S}} / S_{\mathrm{A}}=1$

which relates the air fraction $A$ of humid air to the salinity $S_{\mathrm{A}}$ of seawater at given $w^{\mathrm{A}}$ and $w^{\mathrm{S}}$.

In equilibrium and in the absence of gravity, temperature and pressure in both phases are the same. The vapour-liquid mass ratio $\mathrm{m}^{\mathrm{V}} / \mathrm{m}^{\mathrm{W}}$ of $\mathrm{H}_{2} \mathrm{O}$ in sea air is controlled by the thermodynamic equilibrium condition of equal chemical potentials of water in seawater and water in humid air, Eq. (5.15),
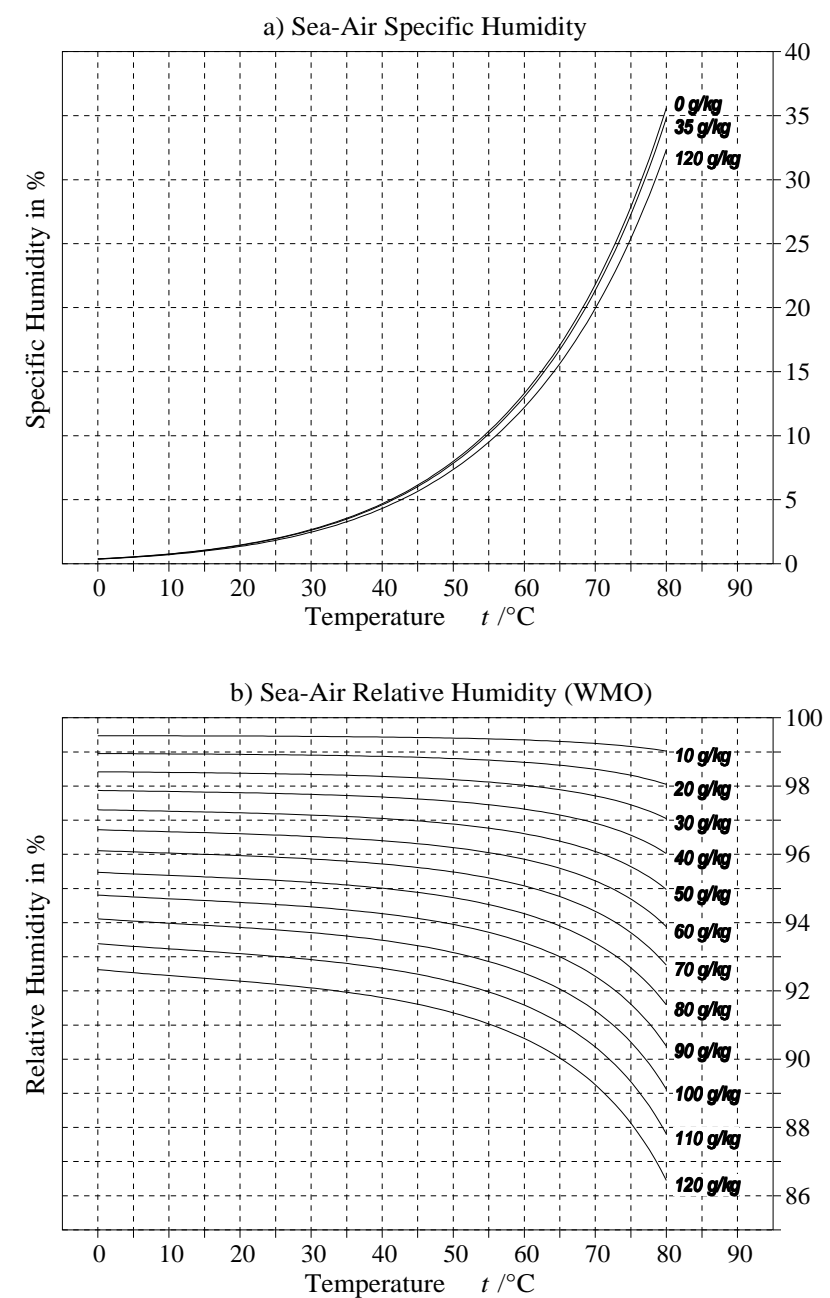

Fig. 3. Specific (a) and relative (b) humidity of sea air, i.e., humid air in equilibrium with seawater, at sea level pressure, $P=101325 \mathrm{~Pa}$, as a function of temperature for different absolute salinities as indicated by the curves. The equilibrium air fraction, $A=A^{\text {cond }}$, at the condensation point is computed from Eq. (6.4), the resulting specific humidity is $q=(1-A)$. The relative humidity $R H_{\text {WMO }}$ is determined from $A$ by the WMO definition (10.4) with the saturated air fraction $A^{\text {sat }}$ from Eq. (7.5). $A^{\text {sat }}$ is displayed as the zero-salinity curve " $0 \mathrm{~g} / \mathrm{kg}$ " in (a).

$g^{\mathrm{AV}}-A\left(\frac{\partial g^{\mathrm{AV}}}{\partial A}\right)_{T, P}=g^{\mathrm{SW}}-S_{\mathrm{A}}\left(\frac{\partial g^{\mathrm{SW}}}{\partial S_{\mathrm{A}}}\right)_{T, P}$.

From Eq. (6.4) along with (6.3), all thermodynamic properties of sea air can be computed as functions of the four independent variables $\left(w^{\mathrm{A}}, w^{\mathrm{S}}, T, P\right)$.

For this purpose, the air fraction $A=A^{\text {cond }}\left(S_{\mathrm{A}}, T, P\right)$ of sea air at equilibrium, i.e. of humid air at the condensation point in contact with seawater, can be computed from the condition (6.4) by Newton iteration. Converted to relative 
humidity using the WMO definition, Eq. (10.4), the subsaturation of sea air as a function of temperature and salinity is shown in Fig. 3 at conditions ranging from cold brackish water to hot concentrated brines. The climatological ambient relative humidity at the ocean surface is about $80 \%$ (Dai, 2006), i.e. clearly below the equilibrium values shown in the lower panel of Fig. 3. The equilibrium specific humidity of sea-air shown in Fig. 3 is the actual quantity to be used in latent heat flow parameterisations (Wells and King-Hele, 1990) that represent the tendency of the system to adjust towards thermodynamic equilibrium.

For a compact writing of formulas related to the equilibrium condition (6.4) of a composite system, we define for sea air a formal phase-transition latency operator $\Lambda_{\mathrm{AS}}[z]$ of the two phases with the subscripts $\mathrm{A}$ for humid air and $\mathrm{S}$ for seawater, with respect to a certain property, $z$, by,

$$
\Lambda_{\mathrm{AS}}[z]=z^{\mathrm{AV}}-A\left(\frac{\partial z^{\mathrm{AV}}}{\partial A}\right)_{T, P}-z^{\mathrm{SW}}+S_{\mathrm{A}}\left(\frac{\partial z^{\mathrm{SW}}}{\partial S_{\mathrm{A}}}\right)_{T, P} .
$$

The operator (6.5) was originally introduced for sea ice in a slightly modified version (Feistel and Hagen, 1998), where it was referred to as the "melting operator". It turns out that for humid air a similar expression is again a very useful tool. The term "latency operator" is used as a natural generalization of "melting operator", applicable for arbitrary phase transitions of the first kind. The operator $\Lambda_{\mathrm{AS}}$ is commutative regarding partial differentiation, $(\partial / \partial x) \Lambda_{\mathrm{AS}}[z]=\Lambda_{\mathrm{AS}}[\partial z / \partial x]$, taken at constant $A$ and $S_{\mathrm{A}}$, if $x$ stands for either $T$ or $P . \Lambda_{\mathrm{AS}}$ is antisymmetric in its indices, $\Lambda_{\mathrm{AS}}[z]=-\Lambda_{\mathrm{SA}}[z]=\Lambda_{\mathrm{SA}}[-z]$. Evidently, in a case of three phases, A, B, C, the related operators obey additivity in the form

$\Lambda_{\mathrm{AB}}=\Lambda_{\mathrm{AC}}+\Lambda_{\mathrm{CB}}$,

corresponding to the transitivity of the binary relation "equilibrium" between phases. Using this operator, the equilibrium condition (6.4) takes the simple form $\Lambda_{\mathrm{AS}}[g]=0$. Its total differential gives the Clausius-Clapeyron differential equation of this phase transition,

$$
\begin{aligned}
& \mathrm{d} \Lambda_{\mathrm{AS}}[g]=0=\left(\frac{\partial}{\partial A} \Lambda_{\mathrm{AS}}[g]\right)_{S_{\mathrm{A}}, T, P} \mathrm{~d} A \\
& +\left(\frac{\partial}{\partial S_{\mathrm{A}}} \Lambda_{\mathrm{AS}}[g]\right)_{A, T, P} \mathrm{~d} S_{\mathrm{A}}+\Lambda_{\mathrm{AS}}\left[\left(\frac{\partial g}{\partial T}\right)_{A, S_{\mathrm{A}}, P}\right] \mathrm{d} T \\
& \quad+\Lambda_{\mathrm{AS}}\left[\left(\frac{\partial g}{\partial P}\right)_{A, S_{\mathrm{A}}, T}\right] \mathrm{d} P
\end{aligned}
$$

which tells us how changes in one state variable must be compensated by changes in some other state variable(s) in order to maintain thermodynamic equilibrium.

Evaluating the first two terms in Eq. (6.7), we define the chemical coefficient $D_{\mathrm{A}}$ of humid air as the derivative of the relative chemical potential with respect to the air fraction change,

$$
D_{\mathrm{A}}=-A\left(\frac{\partial}{\partial A} \Lambda_{\mathrm{AS}}[g]\right)_{S_{\mathrm{A}}, T, P}=A^{2}\left(\partial^{2} g^{\mathrm{AV}} / \partial A^{2}\right)_{T, P},
$$

and $D_{\mathrm{S}}$ of seawater as the derivative with respect to absolute salinity change,

$$
D_{\mathrm{S}}=S_{\mathrm{A}}\left(\frac{\partial}{\partial S_{\mathrm{A}}} \Lambda_{\mathrm{AS}}[g]\right)_{A, T, P}=S_{\mathrm{A}}^{2}\left(\partial^{2} g^{\mathrm{SW}} / \partial S_{\mathrm{A}}^{2}\right)_{T, P} .
$$

From the Second Law of Thermodynamics it follows that the coefficients $D_{\mathrm{A}}$ and $D_{\mathrm{S}}$ are nonnegative (Prigogine and Defay, 1954; Ebeling and Feistel, 1982; De Groot and Mazur, 1984; Landau and Lifschitz, 1987; IOC, 2010). In the idealgas limit, $D_{\mathrm{A}} / T$ is independent of temperature and pressure, Eq. (H15).

Because of total water conservation between the phases, Eq. (6.3), the differentials $\mathrm{d} A$ and $\mathrm{d} S_{\mathrm{A}}$ at constant $w^{\mathrm{A}}$ and $w^{\mathrm{S}}$ of sea air are not independent of each other and are related by

$$
\frac{w^{\mathrm{A}}}{A^{2}} \mathrm{~d} A+\frac{w^{\mathrm{S}}}{S_{\mathrm{A}}^{2}} \mathrm{~d} S_{\mathrm{A}}=0 .
$$

Thus, in Eq. (6.7), $\mathrm{d} A$ can be expressed in terms of $\mathrm{d} S_{\mathrm{A}}$ or vice versa. Eliminating first $\mathrm{d} A$, the Clausius-Clapeyron Eq. (6.7) reads for the total differential $\mathrm{d} S_{\mathrm{A}}$

$$
\frac{w^{\mathrm{S}}}{S_{\mathrm{A}}^{2}} D_{\mathrm{AS}} \mathrm{d} S_{\mathrm{A}}=\Lambda_{\mathrm{AS}}[s] \mathrm{d} T-\Lambda_{\mathrm{AS}}[v] \mathrm{d} P .
$$

Here, the combined chemical air-salt coefficient $D_{\mathrm{AS}}$ is defined as

$D_{\mathrm{AS}}=\frac{A}{w^{\mathrm{A}}} D_{\mathrm{A}}+\frac{S_{\mathrm{A}}}{w^{\mathrm{S}}} D_{\mathrm{S}}=\frac{D_{\mathrm{A}}}{w^{\mathrm{AV}}}+\frac{D_{\mathrm{S}}}{w^{\mathrm{SW}}}$.

The specific entropies of the two phases appearing in $\Lambda_{\mathrm{AS}}[s]$ are $s^{\mathrm{AV}}=-\left(\partial g^{\mathrm{AV}} / \partial T\right)_{A, P}$ and $s^{\mathrm{SW}}=-\left(\partial g^{\mathrm{SW}} / \partial T\right)_{S_{\mathrm{A}}, P}$, and $v^{\mathrm{AV}}=\left(\partial g^{\mathrm{AV}} / \partial P\right)_{A, T}, v^{\mathrm{SW}}=\left(\partial g^{\mathrm{SW}} / \partial P\right)_{S_{\mathrm{A}}, T}$ are the specific volumes of humid air and of seawater in $\Lambda_{\mathrm{AS}}[v]$.

From the exact differential (6.11) we infer the partial derivatives

$\left(\frac{\partial S_{\mathrm{A}}}{\partial T}\right)_{w^{\mathrm{A}}, w^{\mathrm{S}}, P}=\frac{S_{\mathrm{A}}^{2}}{w^{\mathrm{S}}} \frac{\Lambda_{\mathrm{AS}}[s]}{D_{\mathrm{AS}}}$,

and

$\left(\frac{\partial S_{\mathrm{A}}}{\partial P}\right)_{w^{\mathrm{A}}, w^{\mathrm{S}}, T}=-\frac{S_{\mathrm{A}}^{2}}{w^{\mathrm{S}}} \frac{\Lambda_{\mathrm{AS}}[v]}{D_{\mathrm{AS}}}$.

Alternatively, eliminating $\mathrm{d} S_{\mathrm{A}}$ from Eq. (6.7) by means of Eq. (6.10), we get for the corresponding changes of the unsaturated air fraction at the condensation point

$$
\left(\frac{\partial A}{\partial T}\right)_{w^{\mathrm{A}}, w^{\mathrm{s}}, P}=-\frac{A^{2}}{w^{\mathrm{A}}} \frac{\Lambda_{\mathrm{AS}}[s]}{D_{\mathrm{AS}}}
$$


and

$\left(\frac{\partial A}{\partial P}\right)_{w^{\mathrm{A}}, w^{\mathrm{S}}, T}=\frac{A^{2}}{w^{\mathrm{A}}} \frac{\Lambda_{\mathrm{AS}}[v]}{D_{\mathrm{AS}}}$.

With the help of Eqs. (6.13-6.16) we can derive formulas for relevant sea-air properties from temperature and pressure derivatives of the sea-air Gibbs function (6.2),

$g^{\mathrm{SA}}\left(w^{\mathrm{A}}, w^{\mathrm{S}}, T, P\right)=\frac{w^{\mathrm{A}}}{A} g^{\mathrm{AV}}(A, T, P)+\frac{w^{\mathrm{S}}}{S_{\mathrm{A}}} g^{\mathrm{SW}}\left(S_{\mathrm{A}}, T, P\right)$,

where $A$ and $S_{\mathrm{A}}$ are the solutions of the Eqs. (6.3) and (6.4), i.e. functions of $\left(w^{\mathrm{A}}, w^{\mathrm{S}}, T, P\right)$.

The specific entropy of sea air, $s^{\mathrm{SA}}$, is

$s^{\mathrm{SA}}=-\left(\frac{\partial g^{\mathrm{SA}}}{\partial T}\right)_{w^{\mathrm{A}}, w^{\mathrm{S}}, P}=w^{\mathrm{AV}} s^{\mathrm{AV}}+w^{\mathrm{SW}} s^{\mathrm{SW}}$,

and the specific enthalpy of sea air, $h^{\mathrm{SA}}$, is

$h^{\mathrm{SA}}=g^{\mathrm{SA}}+T s^{\mathrm{SA}}=w^{\mathrm{AV}} h^{\mathrm{AV}}+w^{\mathrm{SW}} h^{\mathrm{SW}}$.

Its humid-air parts $s^{\mathrm{AV}}$ and $h^{\mathrm{AV}}$ are plotted in Fig. 4 for different salinities and temperatures at the standard surface air pressure of $101325 \mathrm{~Pa}$.

The specific volume of sea air follows as

$v^{\mathrm{SA}}=\left(\frac{\partial g^{\mathrm{SA}}}{\partial P}\right)_{w^{\mathrm{A}}, w^{\mathrm{S}}, T}=w^{\mathrm{AV}} v^{\mathrm{AV}}+w^{\mathrm{SW}} v^{\mathrm{SW}}$.

Taking the derivatives in Eqs. (6.18) and (6.20), the property of the composite system is just the sum of the fractions of the two phases. Any additional terms cancel because of Eq. (6.4), $\Lambda_{\mathrm{AS}}[g]=0$. This is no longer the case if the second derivatives of the Gibbs function (6.17) are involved, as for the heat capacity, compressibility or thermal expansion.

The specific isobaric heat capacity of sea air, $c_{P}^{\mathrm{SA}}$, is computed from Eq. (6.18), as

$$
\begin{aligned}
& \frac{c_{P}^{\mathrm{SA}}}{T}=\left(\frac{\partial s^{\mathrm{SA}}}{\partial T}\right)_{w^{\mathrm{A}}, w^{\mathrm{S}}, P}=\frac{w^{\mathrm{A}}}{A}\left(\frac{\partial s^{\mathrm{AV}}}{\partial T}\right)_{A, P} \\
& +\left[-\frac{w^{\mathrm{A}}}{A^{2}} s^{\mathrm{AV}}+\frac{w^{\mathrm{A}}}{A}\left(\frac{\partial s^{\mathrm{AV}}}{\partial A}\right)_{T, P}\right]\left(\frac{\partial A}{\partial T}\right)_{w^{\mathrm{A}}, w^{\mathrm{S}}, P} \\
& +\frac{w^{\mathrm{S}}}{S_{\mathrm{A}}}\left(\frac{\partial s^{\mathrm{SW}}}{\partial T}\right)_{S, P}+\left[-\frac{w^{\mathrm{S}}}{S_{\mathrm{A}}^{2}} s^{\mathrm{SW}}+\frac{w^{\mathrm{S}}}{S_{\mathrm{A}}}\left(\frac{\partial s^{\mathrm{SW}}}{\partial S_{\mathrm{A}}}\right)_{T, P}\right] \\
& \left(\frac{\partial S_{\mathrm{A}}}{\partial T}\right)_{w^{\mathrm{A}}, w^{\mathrm{S}}, P}
\end{aligned}
$$

Using Eqs. (6.5), (6.13) and (6.15), this expression can be rearranged to give

$c_{P}^{\mathrm{SA}}=w^{\mathrm{AV}} c_{P}^{\mathrm{AV}}+w^{\mathrm{SW}} c_{P}^{\mathrm{SW}}+T \frac{\left(\Lambda_{\mathrm{AS}}[s]\right)^{2}}{D_{\mathrm{AS}}}$.
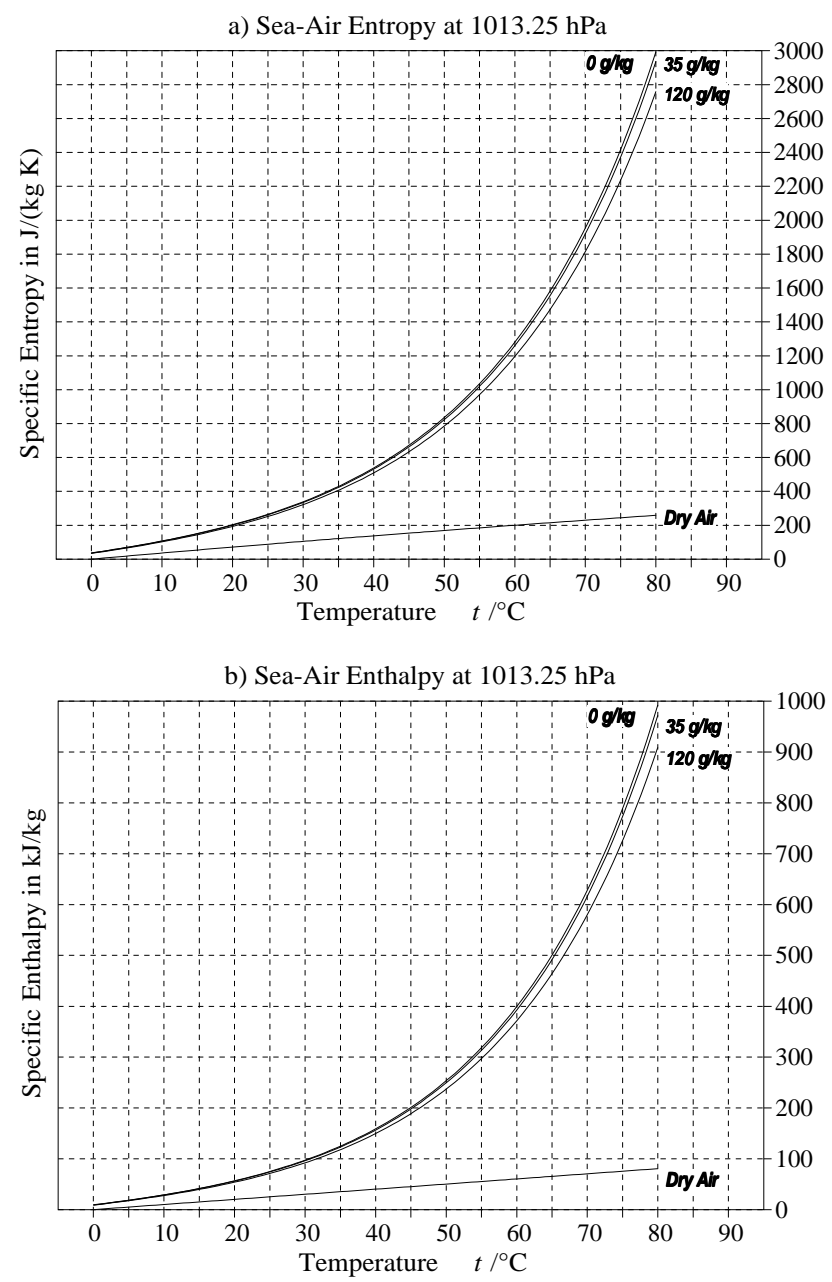

Fig. 4. Entropy $\left.s^{\mathrm{AV}}, \mathbf{a}\right)$, and enthalpy $h^{\mathrm{AV}}, \mathbf{b}$ ), of humid air in equilibrium with seawater, Eq. (6.18), at sea-level pressure as a function of the sea-surface temperature (SST) for salinities 0, 35 and $120 \mathrm{~g} / \mathrm{kg}$. The related humidity is shown in Fig. 3. Entropy and enthalpy of dry air are plotted for comparison.

The last term of Eq. (6.22) is the latent-heat part of the heat capacity caused by the transfer of water between the liquid and the gas phase when the temperature is changing at constant pressure.

For the isothermal compressibility, $\kappa_{T}^{\mathrm{SA}}=$ $-\left(\partial v^{\mathrm{SA}} / \partial P\right)_{w^{\mathrm{A}}, w^{\mathrm{S}}, T} / v^{\mathrm{SA}}$, we repeat similar calculation steps with the result

$$
v^{\mathrm{SA}} \kappa_{T}^{\mathrm{SA}}=w^{\mathrm{AV}} v^{\mathrm{AV}} \kappa_{T}^{\mathrm{AV}}+w^{\mathrm{SW}} v^{\mathrm{SW}} \kappa_{T}^{\mathrm{SW}}+\frac{\left(\Lambda_{\mathrm{AS}}[v]\right)^{2}}{D_{\mathrm{AS}}} .
$$

For the thermal expansion coefficient, $\alpha^{\mathrm{SA}}=$ $\left(\partial v^{\mathrm{SA}} / \partial T\right)_{w^{\mathrm{A}}, w^{\mathrm{s}}, P} / v^{\mathrm{SA}}$, we similarly obtain

$v^{\mathrm{SA}} \alpha^{\mathrm{SA}}=w^{\mathrm{AV}} v^{\mathrm{AV}} \alpha^{\mathrm{AV}}+w^{\mathrm{SW}} v^{\mathrm{SW}} \alpha^{\mathrm{SW}}+\frac{\Lambda_{\mathrm{AS}}[v] \Lambda_{\mathrm{AS}}[s]}{D_{\mathrm{AS}}}$. 
Various other thermodynamic properties of sea air are available from these coefficients using standard thermodynamic relations. As an example, a property of particular interest in meteorology is the adiabatic lapse rate, $\Gamma^{\mathrm{SA}}$, describing the temperature change with pressure under isentropic conditions, similar to Eq. (5.25),

$$
\Gamma^{\mathrm{SA}}=\left(\frac{\partial T}{\partial P}\right)_{w^{\mathrm{A}}, w^{\mathrm{S}, s^{\mathrm{AS}}}}=-\frac{\left(\partial^{2} g^{\mathrm{SA}} / \partial T \partial P\right)_{w^{\mathrm{A}}, w^{\mathrm{S}}}}{\left(\partial^{2} g^{\mathrm{SA}} / \partial T^{2}\right)_{w^{\mathrm{A}}, w^{\mathrm{S}}, P}} .
$$

From Eqs. (6.18), (6.20-6.22) and (6.24) we get

$$
\Gamma^{\mathrm{SA}}=\frac{w^{\mathrm{AV}} v^{\mathrm{AV}} \alpha^{\mathrm{AV}}+w^{\mathrm{SW}} v^{\mathrm{SW}} \alpha^{\mathrm{SW}}+\Lambda_{\mathrm{AS}}[v] \Lambda_{\mathrm{AS}}[s] / D_{\mathrm{AS}}}{\left(w^{\mathrm{AV}} c_{P}^{\mathrm{AV}}+w^{\mathrm{SW}} c_{P}^{\mathrm{SW}}\right) / T+\left(\Lambda_{\mathrm{AS}}[s]\right)^{2} / D_{\mathrm{AS}}}
$$

for air over seawater reacting to air pressure variations with warming or cooling caused by adiabatic expansion as well as condensation or evaporation.

To obtain an appropriate expression for the latent heat, $L_{P}^{\mathrm{AS}}$, of isobaric evaporation from the sea surface, we divide the latent heat-capacity term, $T \Lambda_{\mathrm{AS}}[s]^{2} / D_{\mathrm{AS}}$, from Eq. (6.22) by the related isobaric evaporation rate, Eq. (6.15),

$$
\left(\frac{\partial w^{\mathrm{AV}}}{\partial T}\right)_{w^{\mathrm{A}}, w^{\mathrm{s}}, P}=-\frac{w^{\mathrm{A}}}{A^{2}}\left(\frac{\partial A}{\partial T}\right)_{w^{\mathrm{A}}, w^{\mathrm{s}}, P}=\frac{\Lambda_{\mathrm{AS}}[s]}{D_{\mathrm{AS}}} .
$$

Under consideration of $\Lambda_{\mathrm{AS}}[g]=0$, Eq. (6.4), we eventually arrive at the formula for the latent heat,

$$
\begin{gathered}
L_{P}^{\mathrm{SA}}=T \Lambda_{\mathrm{AS}}[s]=\Lambda_{\mathrm{AS}}[h] \equiv h^{\mathrm{AV}}-A\left(\frac{\partial h^{\mathrm{AV}}}{\partial A}\right)_{T, P} \\
-h^{\mathrm{SW}}+S_{\mathrm{A}}\left(\frac{\partial h^{\mathrm{SW}}}{\partial S_{\mathrm{A}}}\right)_{T, P} .
\end{gathered}
$$

This expression does not depend on the absolute enthalpies of $\mathrm{H}_{2} \mathrm{O}$, air, and sea salt. On the contrary, the simple enthalpy difference, $\Delta h=h^{\mathrm{AV}}-h^{\mathrm{SW}}$, between the gaseous and the liquid component depends on the arbitrary values of the absolute enthalpies of air and of salt. Only in the special case of pure water, $S_{\mathrm{A}}=0, A=0$, does this difference equal the latent heat of evaporation (Kirchhoff's law).

From Eq. (6.28) it is reasonable to refer to $h^{\mathrm{AV}}-A h_{A}^{\mathrm{AV}}$ as the specific enthalpy of water in humid air, and $h^{\mathrm{SW}}-S_{\mathrm{A}} h_{S_{\mathrm{A}}}^{\mathrm{SW}}$ as the specific enthalpy of water in seawater, or the partial specific enthalpies (Glasstone, 1947; Pruppacher and Klett, 1997), Eqs. (3.7) and (5.19). In the ideal-gas limit, $h^{\mathrm{AV}}-A h_{A}^{\mathrm{AV}}$ equals the enthalpy of vapour, Eq. (H12), as one would expect. The latent heat, Eq. (6.28), is an almost linear function of temperature and depends only weakly on salinity, air fraction and pressure (Fig. 5). For illustration we give the values for the standard ocean surface state $\left(T_{\mathrm{SO}}=273.15 \mathrm{~K}, P_{\mathrm{SO}}=101325 \mathrm{~Pa}, S_{\mathrm{SO}}=35.16504 \mathrm{~g} \mathrm{~kg}^{-1}\right)$. At this state, the air fraction of sea-air is $A_{\mathrm{SO}}=0.996293$, the saturated air fraction would be $A^{\text {sat }}=0.996223$, i.e. the relative humidity of sea-air is, from Eq. (10.4), $R H_{\mathrm{WMO}}=98.14 \%$, Fig. 3. The latent heat is $L_{P}^{\mathrm{SA}}=$ $2498510 \mathrm{Jkg}^{-1}$, its derivatives are $\left(\partial L_{P}^{\mathrm{SA}} / \partial S_{\mathrm{A}}\right)_{A, T, P}=$ $-30775 \mathrm{Jkg}^{-1},\left(\partial L_{P}^{\mathrm{SA}} / \partial T\right)_{A, S_{\mathrm{A}}, P}=-2379 \mathrm{Jkg}^{-1} \mathrm{~K}^{-1}$ and $\left(\partial L_{P}^{\mathrm{SA}} / \partial P\right)_{A, S_{\mathrm{A}}, T}=-0.0136 \mathrm{Jkg}^{-1} \mathrm{~Pa}^{-1}$.

\section{Properties of wet and saturated humid air}

As an important special case, at zero salinity, $w^{\mathrm{S}}=0$, the Gibbs function (6.2) describes the properties of wet air, i.e. saturated air combined with liquid water, e.g. in the form of droplets as in clouds or fog. It is therefore often denoted as "cloudy air" in meteorology. However, this Gibbs function for a composite system of liquid water and humid air in mutual equilibrium may also be applied to e.g. saturated air resting over a fresh water lake, or to the precipitation of dew. Below the freezing point of water, in the formulas derived here the properties of liquid water must be replaced with those of ice.

The Gibbs function of wet air, $g^{\mathrm{AW}}$, follows from Eq. (6.2) as

$$
\begin{aligned}
& g^{\mathrm{AW}}\left(w^{\mathrm{A}}, T, P\right)=g^{\mathrm{SA}}\left(w^{\mathrm{A}}, 0, T, P\right) \\
& =w^{\mathrm{AV}} g^{\mathrm{AV}}(A, T, P)+w^{\mathrm{W}} g^{\mathrm{SW}}(0, T, P)
\end{aligned}
$$

and the related latency operator reads

$$
\Lambda_{\mathrm{AW}}[z]=z^{\mathrm{AV}}-A\left(\frac{\partial z^{\mathrm{AV}}}{\partial A}\right)_{T, P}-z^{\mathrm{W}} .
$$

The mass fractions are $w^{\mathrm{AV}}=$ $\left(m^{\mathrm{A}}+m^{\mathrm{V}}\right) /\left(m^{\mathrm{A}}+m^{\mathrm{V}}+m^{\mathrm{W}}\right)$ for the humid air in the wet air, $w^{\mathrm{W}}=m^{\mathrm{W}} /\left(m^{\mathrm{A}}+m^{\mathrm{V}}+m^{\mathrm{W}}\right)$ for the liquid water in the wet air, and $w^{\mathrm{A}}=m^{\mathrm{A}} /\left(m^{\mathrm{A}}+m^{\mathrm{V}}+m^{\mathrm{W}}\right), A=$ $m^{\mathrm{A}} /\left(m^{\mathrm{A}}+m^{\mathrm{V}}\right)$ for the dry air content, which together obey mass conservation of water, $m^{\mathrm{V}}+m^{\mathrm{W}}$, between vapour and liquid, i.e. the identity

$$
\frac{w^{\mathrm{A}}}{A}+w^{\mathrm{W}}=w^{\mathrm{AV}}+w^{\mathrm{W}}=1 .
$$

Thus, the Gibbs function (7.1) can be written in the form

$$
\begin{aligned}
& g^{\mathrm{AW}}\left(w^{\mathrm{A}}, T, P\right)=\frac{w^{\mathrm{A}}}{A(T, P)} g^{\mathrm{AV}}(A, T, P) \\
& +\left(1-\frac{w^{\mathrm{A}}}{A(T, P)}\right) g^{\mathrm{W}}(T, P)
\end{aligned}
$$

as a linear function of the given air fraction, $w^{\mathrm{A}}$, of the wet air.

As a function of $T$ and $P$, the saturated air fraction of humid air, $A_{\mathrm{AW}}^{\mathrm{sat}}(T, P)$, is obtained from the equilibrium condition

$$
\Lambda_{\mathrm{AW}}[g]=g^{\mathrm{AV}}-A\left(\frac{\partial g^{\mathrm{AV}}}{\partial A}\right)_{T, P}-g^{\mathrm{W}}=0 .
$$




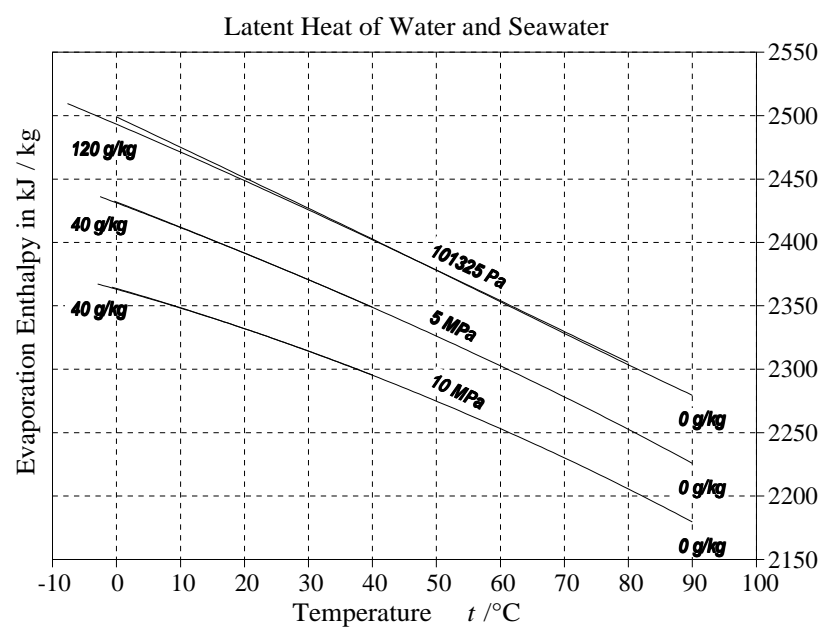

Fig. 5. Isobaric evaporation enthalpy of water, indicated by " $0 \mathrm{~g} / \mathrm{kg}$ ", and of seawater with salinity $120 \mathrm{~g} \mathrm{~kg}^{-1}$ at $101325 \mathrm{~Pa}$ and $40 \mathrm{~g} \mathrm{~kg}^{-1}$ at $5 \mathrm{MPa}$ and $10 \mathrm{MPa}$, computed from Eq. (6.28). The salinity dependence is very weak in either case. At high pressure the validity of the current Gibbs function of seawater (3.1) is restricted to maxima of only $40 \mathrm{~g} \mathrm{~kg}^{-1}$ and $40^{\circ} \mathrm{C}$, but at atmospheric pressure it is valid up to $120 \mathrm{~g} \mathrm{~kg}^{-1}$ and $80^{\circ} \mathrm{C}$ (IAPWS, 2008; Feistel, 2008). The lower temperature bounds are the particular freezing points of water or seawater.

Throughout this section, if not explicitly stated otherwise, $A$ equals the saturation value $A_{\mathrm{AW}}^{\mathrm{sat}}$, Fig. 8, since humid air in equilibrium with liquid water is always saturated.

The function $A_{\mathrm{AW}}^{\mathrm{sat}}(T, P)$ is different from the saturation $A_{\mathrm{AI}}^{\text {sat }}(T, P)$ of humid air with respect to ice, Sect. 8. Both functions take the same values on the freezing curve because $g^{\mathrm{W}}$ equals $g^{\text {Ih }}$ under the equilibrium conditions, Eq. (7.5) and its counterpart for ice air, Eq. (8.7).

Solving Eq. (7.5) for either $T(A, P)$ or $P(A, T)$ provides the saturation point (SP) temperature $T_{\mathrm{SP}}$ or pressure $P_{\mathrm{SP}}$, also known as the isentropic condensation level (ICL), i.e. the saturation level for an unsaturated parcel lifted moistadiabatically (Stull, 2003; Jacobson, 2005). This is considered in more detail below, see Eq. (7.25) and following.

The total differential of the phase equilibrium condition (7.5) is the related Clausius-Clapeyron differential equation,

$$
\begin{aligned}
& \mathrm{d} \Lambda_{\mathrm{AW}}[g]=0=\left(\frac{\partial \Lambda_{\mathrm{AW}}[g]}{\partial A}\right)_{T, P} \mathrm{~d} A \\
& +\Lambda_{\mathrm{AW}}\left[\left(\frac{\partial g}{\partial T}\right)_{A, P}\right] \mathrm{d} T+\Lambda_{\mathrm{AW}}\left[\left(\frac{\partial g}{\partial P}\right)_{A, T}\right] \mathrm{d} P,
\end{aligned}
$$

which represents a special case of Eq. (6.7).

Commonly, the Clausius-Clapeyron differential equation for a two-phase system such as water-vapour or ice-vapour takes the form $\mathrm{d} P / \mathrm{d} T=\Delta s / \Delta v$, obtained by taking the total differential of the equilibrium condition of equal chemical potentials of the two phases, e.g., Eq. (8.1). An approximate analytical solution $P(T)$ can be derived analytically and represents a reasonable estimate, for example, for the sublimation curve of ice (Feistel and Wagner, 2007). In the special case when no air is present, $A=0, \mathrm{~d} A=0$, the generalized form, Eq. (7.6), reduces to the familiar Clausius-Clapeyron equation for the liquid-vapour transition. Geometrically, any displacement ( $\mathrm{d} A, \mathrm{~d} T, \mathrm{~d} P$ ) which obeys Eq. (7.6) is tangential to the phase transition surface defined by Eq. (7.5) in the $(A, T, P)$ phase space, while the coefficients of $(\mathrm{d} A, \mathrm{~d} T$, $\mathrm{d} P$ ) in Eq. (7.6) form a "normal vector".

Reading Eq. (7.6) as an exact differential for $\mathrm{d} A$, we infer for the partial derivatives of $A$, i.e., for changes of the air fraction with temperature,

$\delta_{P}^{\mathrm{AW}}=-\left(\frac{\partial A_{\mathrm{AW}}^{\mathrm{sat}}}{\partial T}\right)_{P}=A_{\mathrm{AW}}^{\mathrm{sat}} \frac{\Lambda_{\mathrm{AW}}[s]}{D_{\mathrm{A}}}$,

or pressure,

$\delta_{T}^{\mathrm{AW}}=-\left(\frac{\partial A_{\mathrm{AW}}^{\mathrm{sat}}}{\partial P}\right)_{T}=-A_{\mathrm{AW}}^{\mathrm{sat}} \frac{\Lambda_{\mathrm{AW}}[v]}{D_{\mathrm{A}}}$,

due to condensation or evaporation. The coefficient $D_{\mathrm{A}}$ is defined in Eq. (6.8). The right-hand sides of Eqs. (7.7) and (7.8) depend only on the air fraction $A$ of the humid-air component rather than on the air fraction $w^{\mathrm{A}}$ of the total sample, i.e. they are independent of the mass of liquid water present. They can therefore be regarded as the isothermal and isobaric drying rates of humid air, i.e. the decrease of its saturated air fraction $A_{\mathrm{AW}}^{\mathrm{sat}}$ due to heating or compression, corresponding to a lowering of the relative humidity at constant specific humidity of an unsaturated sample at constant $P$ or $T$, respectively. Below the freezing point, different drying rates $\delta^{\mathrm{AI}}$ must be applied with respect to the sublimation equilibrium, Sect. 8 .

From Eqs. (7.7), (7.8), the adiabatic drying rate of humid air with respect to increasing pressure is computed by means of the chain rule as

$\delta_{s}^{\mathrm{AW}}=-\left(\frac{\partial A_{\mathrm{AW}}^{\mathrm{sat}}}{\partial P}\right)_{s}=\delta_{T}^{\mathrm{AW}}+\Gamma^{\mathrm{AV}} \delta_{P}^{\mathrm{AW}}$.

Here, the adiabatic lapse rate of humid air, $\Gamma^{\mathrm{AV}}=$ $(\partial T / \partial P)_{A, s}$, is given by Eq. (5.25).

Returning to wet air, we find for the entropy, Eq. (6.18),

$s^{\mathrm{AW}}\left(w^{\mathrm{A}}, T, P\right)=-\left(\frac{\partial g^{\mathrm{AW}}}{\partial T}\right)_{w^{\mathrm{A}}, P}=w^{\mathrm{AV}} s^{\mathrm{AV}}+w^{\mathrm{W}} s^{\mathrm{W}}$,

and the specific volume, Eq. (6.20),

$v^{\mathrm{AW}}\left(w^{\mathrm{A}}, T, P\right)=\left(\frac{\partial g^{\mathrm{AW}}}{\partial P}\right)_{w^{\mathrm{A}}, T}=w^{\mathrm{AV}} v^{\mathrm{AV}}+w^{\mathrm{W}} v^{\mathrm{W}}$.

The entropy of wet air, Eq. (7.10), is plotted in Fig. 6 ("tephigram") as a function of temperature, pressure and the dryair fraction $w^{\mathrm{A}}$ between the freezing point and complete 
evaporation of the liquid component. Evidently, the temperature range of validity of Eq. (7.10) depends strongly on the pressure and the air fraction, given by the condition $w^{\mathrm{A}} \leq A_{\mathrm{AW}}^{\text {sat }}(T, P)$ from Eq. (7.3) and $A=A_{\mathrm{AW}}^{\mathrm{sat}}(T, P)$ computed from Eq. (7.5), as shown in Fig. 8.

Similar to Eq. (5.20), the potential temperature of wet air follows from the equation,

$s^{\mathrm{AW}}\left(w^{\mathrm{A}}, \theta^{\mathrm{AW}}, P_{0}\right)=s^{\mathrm{AW}}\left(w^{\mathrm{A}}, T, P\right)$.

or, equivalently, from the enthalpy, $h^{\mathrm{AW}}$,

$h^{\mathrm{AW}}=g^{\mathrm{AW}}-T\left(\frac{\partial g^{\mathrm{AW}}}{\partial T}\right)_{w^{\mathrm{A}}, P}$

as its isobaric entropy derivative (Feistel and Hagen, 1995; Feistel, 2008a) taken at the in-situ entropy $s$ and the surface pressure $P_{0}$ as the reference pressure,

$\theta^{\mathrm{AW}}=\left(\frac{\partial h^{\mathrm{AW}}}{\partial s}\right)_{w^{\mathrm{A}}, P=P_{0}}$.

Equation (7.14) is the formal solution of the implicit Eq. (7.12) for $\theta^{\mathrm{AW}}$ which can be inferred from the total differential of the enthalpy potential function, $h^{\mathrm{AW}}\left(w^{\mathrm{A}}, s, P\right)$, obtained from Eqs. (7.13) and (7.5), as,

$\mathrm{d} h^{\mathrm{AW}}=\mu^{\mathrm{AV}} \mathrm{d} w^{\mathrm{A}}+T \mathrm{~d} s+v \mathrm{~d} P$,

where the relative chemical potential, $\mu^{\mathrm{AV}}=\mu^{\mathrm{A}}-\mu^{\mathrm{V}}$, of humid air is given by Eqs. (5.14), (5.15).

Equations (7.12) and (7.14) are meaningful only if liquid water is still present at the reference pressure $P_{0}$, i.e. if the dewpoint pressure (ICL) is higher than $P_{0}$. Otherwise, one has to insert the conserved entropy $s=s^{\mathrm{AW}}$ computed from Eq. (7.10) and the conserved air fraction $A=w^{\mathrm{A}}$ into the enthalpy $h^{\mathrm{AV}}\left(A, s, P_{0}\right)$ of unsaturated humid air and compute the related potential temperature from Eq. (5.21).

In analogy to Eqs. (6.22-6.28) we compute the heat capacity, as,

$$
\begin{gathered}
c_{P}^{\mathrm{AW}}=T\left(\frac{\partial s^{\mathrm{AW}}}{\partial T}\right)_{w^{\mathrm{A}}, P}=w^{\mathrm{AV}} c_{P}^{\mathrm{AV}}+w^{\mathrm{W}} c_{P}^{\mathrm{W}} \\
+w^{\mathrm{AV}} \frac{T\left(\Lambda_{\mathrm{AW}}[s]\right)^{2}}{D_{\mathrm{A}}},
\end{gathered}
$$

the isothermal compressibility, $\kappa_{T}^{\mathrm{AW}}$,

$$
\begin{aligned}
& v^{\mathrm{AW}} \kappa_{T}^{\mathrm{AW}}=-\left(\frac{\partial v^{\mathrm{AW}}}{\partial P}\right)_{w^{\mathrm{A}}, T}=w^{\mathrm{AV}} v^{\mathrm{AV}} \kappa_{T}^{\mathrm{AV}}+w^{\mathrm{W}} v^{\mathrm{W}} \kappa_{T}^{\mathrm{W}} \\
& +w^{\mathrm{AV}} \frac{\left(\Lambda_{\mathrm{AW}}[v]\right)^{2}}{D_{\mathrm{A}}},
\end{aligned}
$$

and the thermal expansion coefficient, $\alpha^{\mathrm{AW}}$,

$$
\begin{gathered}
v^{\mathrm{AW}} \alpha^{\mathrm{AW}}=\left(\frac{\partial v^{\mathrm{AW}}}{\partial T}\right)_{w^{\mathrm{A}}, P}=w^{\mathrm{AV}} v^{\mathrm{AV}} \alpha^{\mathrm{AV}}+w^{\mathrm{W}} v^{\mathrm{W}} \alpha^{\mathrm{W}} \\
+w^{\mathrm{AV}} \frac{\Lambda_{\mathrm{AW}}[v] \Lambda_{\mathrm{AS}}[s]}{D_{\mathrm{A}}} .
\end{gathered}
$$
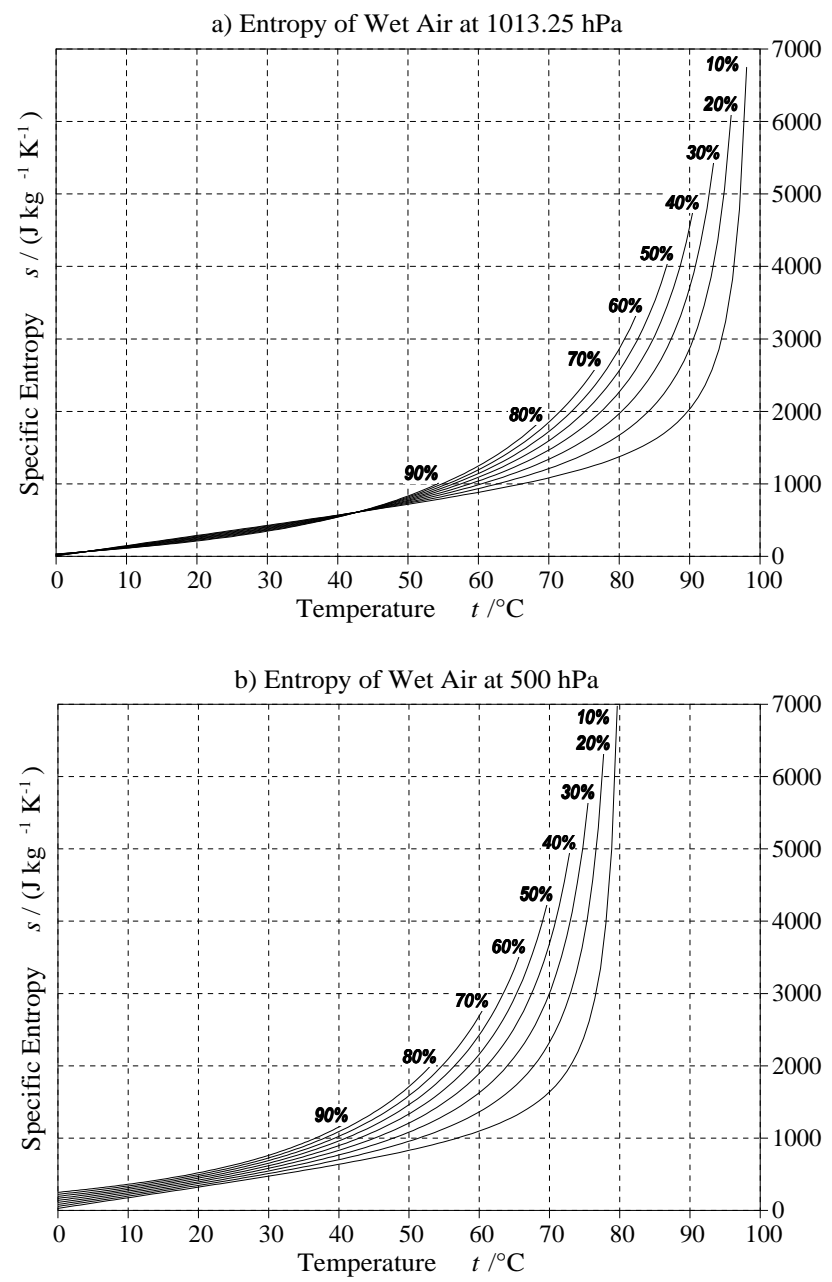

Fig. 6. Entropy of wet air, Eq. (7.10), as a function of temperature at $1013.25 \mathrm{hPa}$ (a) and $500 \mathrm{hPa}$ (b) between the freezing point and complete evaporation of the liquid component for dry-air fractions $w^{\mathrm{A}}$ of wet air, Eq. (7.3), between $10 \%$ and $90 \%$ as indicated by the curves. The related liquid mass fractions $w^{\mathrm{W}}$ of wet air, Eq. (7.3), are shown in Fig. 9.

The adiabatic lapse rate of wet air, often regarded as the "saturated" lapse rate, is computed from Eqs. (7.16) and (7.18) as

$$
\begin{aligned}
& \Gamma^{\mathrm{AW}}=\left(\frac{\partial T}{\partial P}\right)_{w^{\mathrm{A}}, s} \\
& =\frac{w^{\mathrm{AV}} v^{\mathrm{AV}} \alpha^{\mathrm{AV}}+w^{\mathrm{W}} v^{\mathrm{W}} \alpha^{\mathrm{W}}+w^{\mathrm{AV}} \Lambda_{\mathrm{AW}}[v] \Lambda_{\mathrm{AW}}[s] / D_{\mathrm{A}}}{\left(w^{\mathrm{AV}} c_{P}^{\mathrm{AV}}+w^{\mathrm{W}} c_{P}^{\mathrm{W}}\right) / T+w^{\mathrm{AV}}\left(\Lambda_{\mathrm{AW}}[s]\right)^{2} / D_{\mathrm{A}}} .
\end{aligned}
$$

Neglecting the liquid contributions to the heat capacity in the denominator, i.e., using $w^{\mathrm{W}} c_{P}^{\mathrm{W}} \approx 0$, leads to the approximate "pseudoadiabatic" lapse rate (Emanuel, 1994; Jacobson, 2005). In this simplified model the condensate is assumed to precipitate and disappear immediately from the rising parcel (v. Bezold, 1888; Rogers and Yau, 1989). This model picture fails for sinking air as it cannot explain the 


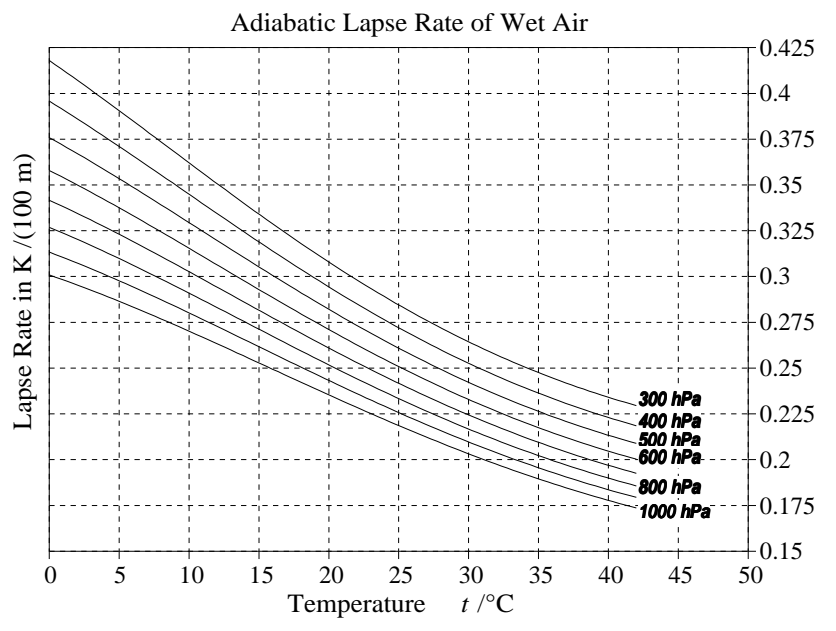

Fig. 7. Adiabatic lapse rate (7.19) of wet air at the dewpoint, i.e. containing a negligible amount of liquid water, at different temperatures and pressures as indicated by the curves. Note that this lapse rate is termed "moist" in some literature. For convenience, the rate is shown per $100 \mathrm{~m}$ altitude difference as common in meteorology, rather than per pressure difference. For comparison, the lapse rate of humid air is shown in Fig. 2.

latent contributions in Eq. (7.19) since there is then no liquid in the parcel that could evaporate. If in reality precipitation happens during rising, the sinking air must correctly be described by the moist-adiabatic lapse rate (5.25) rather than Eq. (7.19), which produces the warming "foehn wind" effect at the lee side of a mountain. In addition to being convenient simplifications, the definition of such "pseudo" properties is practically useful for cases in which the condensed water fraction is unknown, such as for measured radiosonde profiles. Selected values of Eq. (7.19) computed at a negligible condensate fraction are displayed in Fig. 7.

During the adiabatic lifting process, the liquid fraction increases at the adiabatic condensation rate

$-\left(\frac{\partial w^{\mathrm{W}}}{\partial P}\right)_{w^{\mathrm{A}}, s}=\left(1-w^{\mathrm{W}}\right) \frac{\delta_{\mathrm{S}}^{\mathrm{AW}}}{A}$.

The latent heat for the evaporation of liquid water follows from Eq. (7.16) as the ratio of the latent part of the heat capacity to the isobaric evaporation rate of the liquid fraction (Fig. 9),

$-\left(\frac{\partial w^{\mathrm{W}}}{\partial T}\right)_{w^{\mathrm{A}}, P}=\frac{w^{\mathrm{A}}}{A} \frac{\Lambda_{\mathrm{AW}}[s]}{D_{\mathrm{A}}}$,

derived from Eqs. (7.3) and (7.7), as

$L_{P}^{\mathrm{AW}}=T \Lambda_{\mathrm{AW}}[s]=\Lambda_{\mathrm{AW}}[h] \equiv h^{\mathrm{AV}}-A\left(\frac{\partial h^{\mathrm{AV}}}{\partial A}\right)_{T, P}-h^{\mathrm{W}}(7.22)$

Of particular interest is the saturation point of humid air. At the dewpoint, no liquid water is present, $w^{\mathrm{W}}=0$, so we infer from Eq. (7.3) the relation

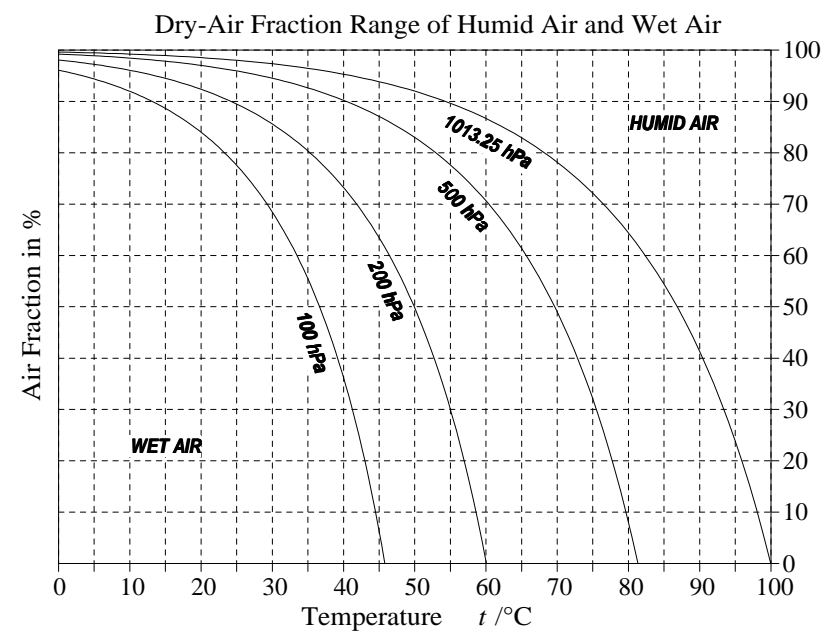

Fig. 8. The saturation curves $A_{\mathrm{AW}}^{\mathrm{sat}}(T, P)$ computed from solving Eq. (7.5) at the pressures 1013.25, 500, 200 and $100 \mathrm{hPa}$, as indicated, between the freezing and the boiling temperature of pure water separate the physically sensible dry-air fractions, $A \geq$ $A_{\mathrm{AW}}^{\mathrm{sat}}(T, P)$, of the single-phase state of humid air from those of the two-phase state of wet air, $w^{\mathrm{A}} \leq A_{\mathrm{AW}}^{\mathrm{sat}}(T, P)$. On a particular saturation curve the two systems coincide, Eqs. (7.23), (7.24). The liquid fraction of wet air, $w^{\mathrm{W}}=1-w^{\mathrm{A}} / A_{\mathrm{AW}}^{\mathrm{sat}}(T, P)$, Eq. (7.3), is shown in Fig. 9.

$w^{\mathrm{A}}=A_{\mathrm{AW}}^{\mathrm{sat}}(T, P)$ at the dewpoint

between the independent variables of Eq. (7.4). This dewpoint surface in the $\left(w^{\mathrm{A}}, T, P\right)$ space bounds the validity of the Gibbs function of wet air. On the surface, the Gibbs functions of wet air and of humid air have the same values,

$g^{\mathrm{WA}}\left(w^{\mathrm{A}}, T, P\right)=g^{\mathrm{AV}}\left(A_{\mathrm{AW}}^{\mathrm{sat}}, T, P\right)$.

For an unsaturated humid-air parcel with the in-situ properties $(A, T, P)$, the dewpoint temperature $T_{\mathrm{DP}}$ and pressure $P_{\mathrm{DP}}$ are met during adiabatic uplift when the two equations

$A_{\mathrm{AW}}^{\mathrm{sat}}\left(T_{\mathrm{DP}}, P_{\mathrm{DP}}\right)=A$

$s^{\mathrm{AV}}\left(A, T_{\mathrm{DP}}, P_{\mathrm{DP}}\right)=s^{\mathrm{AV}}(A, T, P)$

are satisfied. The solution of Eqs. (7.25) and (7.26) defines the adiabatic dewpoint temperature $T_{\mathrm{DP}}(A, T, P)$ and pressure $P_{\mathrm{DP}}(A, T, P)$ for a given unsaturated sample of humid air. This dewpoint temperature is also regarded as the isentropic condensation temperature (ICT), defined as the temperature at which saturation is reached when unsaturated air is cooled adiabatically at a constant mass mixing ratio of water vapour (Rogers and Yau, 1989; Jacobson, 2005). The dewpoint pressure is regarded as the isentropic condensation 


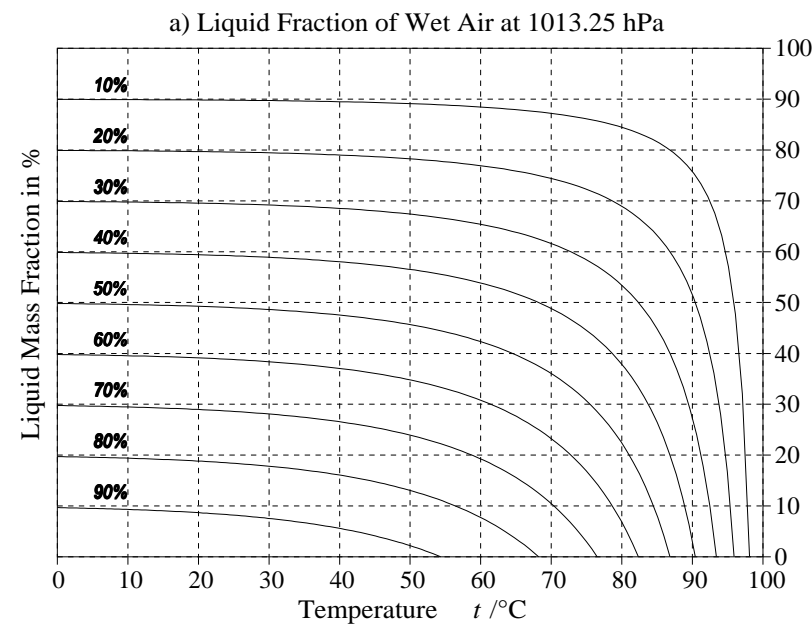

b) Liquid Fraction of Wet Air at $500 \mathrm{hPa}$

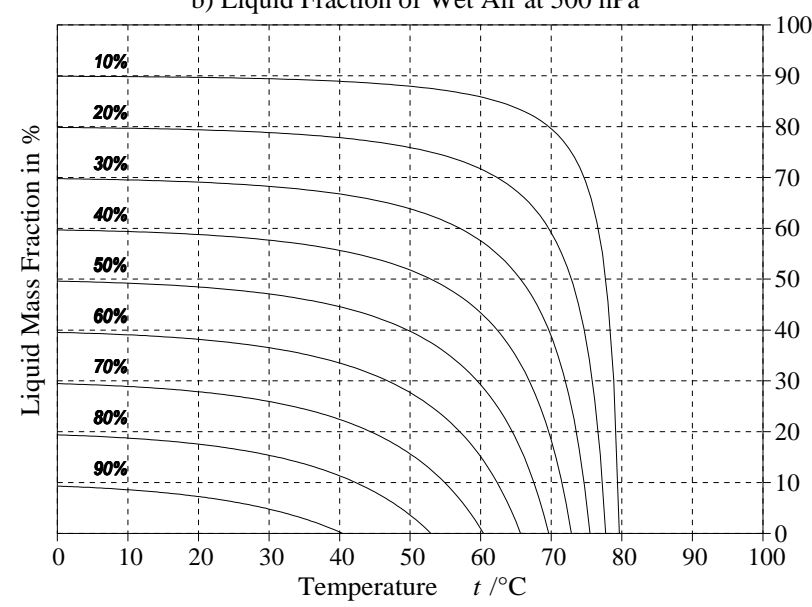

Fig. 9. Liquid mass fraction $w^{\mathrm{W}}$ of wet air, the "burden" of condensed water, as a function of temperature at $1013.25 \mathrm{hPa}$ (a) and $500 \mathrm{hPa}(\mathbf{b})$ between the freezing point and the point at which complete evaporation of the liquid component occurs for dry-air fractions $w^{\mathrm{A}}$ of wet air, Eq. (7.3), between $10 \%$ and $90 \%$ as indicated by the curves. The complement $\left(1-w^{\mathrm{W}}\right)$ is the gaseous humid-air fraction of wet air.

level (ICL) during adiabatic uplift. As an example, solutions of Eqs. (7.25), (7.26) are shown in Fig. 16.

Approaching the transition point from the other side (i.e., from below the saturation curve in Fig. 8), this dewpoint ("cloud base") is reached by adiabatic compression of wet air with the in-situ properties $\left(w^{\mathrm{A}}, T, P\right)$ if the conditions

$A_{\mathrm{AW}}^{\mathrm{sat}}\left(T_{\mathrm{DP}}, P_{\mathrm{DP}}\right)=w^{\mathrm{A}}$

$s^{\mathrm{WA}}\left(w^{\mathrm{A}}, T_{\mathrm{DP}}, P_{\mathrm{DP}}\right)=s^{\mathrm{WA}}\left(w^{\mathrm{A}}, T, P\right)$

hold, which define the point where the parcel's liquid fraction $w^{\mathrm{W}}$ is completely evaporated but the humid air is still saturated (Fig. 9). Thus, passing the dewpoint adiabatically, entropy, Gibbs energy and therefore also enthalpy cross over continuously between humid air and wet air. Since the mass fraction $w^{\mathrm{W}}$ and thus the volume of the native liquid phase are zero at the saturation point, the density changes continuously, too, and therefore also the Helmholtz function and the internal energy of the parcel. The second derivatives of the potential functions such as the compressibility or the heat capacity change discontinuously across the saturation curve, however.

Property changes of wet air subject to adiabatic processes are preferably computed from the enthalpy as the appropriate thermodynamic potential. To express the enthalpy $h^{\mathrm{AW}}$, Eq. (7.13), in terms of its natural variables $w^{\mathrm{A}}, s$ and $P$, the Eq. (7.12),

$s^{\mathrm{AW}}\left(w^{\mathrm{A}}, T, P\right)=s$

derived from the Gibbs function (7.4) must be inverted numerically for the temperature, $T\left(w^{\mathrm{A}}, s, P\right)$. At given $w^{\mathrm{A}}$ and $P$, this computation is sensible only if $s$ takes values restricted to its particular range of validity, $s_{\min }\left(w^{\mathrm{A}}, P\right) \leq s \leq$ $s_{\max }\left(w^{\mathrm{A}}, P\right)$, as shown in Fig. 6 , bound between freezing and complete evaporation of the liquid part. From the given pressure $P$, the minimum temperature $T_{\min }$ is available from the freezing point of liquid water, Eq. (8.1), and from it, in turn, the minimum entropy of wet air, $s_{\min }=s^{\mathrm{AW}}\left(w^{\mathrm{A}}, T_{\min }\right.$, $P)$, Eq. (7.10). The upper end point, the "cloud base", is defined by the saturation of humid air at the given air fraction, $A=w^{\mathrm{A}}$, Eq. (7.27), solving Eq. (7.5) for $T=T_{\max }$ at the given pressure $P$. Since entropy is continuous at the phasetransition point from humid to wet air, the maximum entropy of wet air is therefore given by $s_{\max }=s^{\mathrm{AV}}\left(w^{\mathrm{A}}, T_{\max }, P\right)$, Eq. (5.16).

\section{Properties of sea air below the freezing point}

At temperatures below the freezing point of seawater, the sea-air system forms a third phase, solid ice Ih, which does not contain relevant amounts of sea salt or air. More precisely, ice-crystal distortions due to occasionally built-in salt or air particles are considered as negligibly seldom, which can safely be expected if the freezing process is sufficiently slow or the ice is sufficiently aged. This assumption does not exclude the presence of macroscopic brine pockets or air bubbles within the ice which count as parts of the liquid or the gas phase, respectively. In other words, the equilibrium properties between humid air, seawater and ice do no depend on the spatial distributions or shapes of the three phases in the system. Any surface-tension effects are neglected in this model.

The binary relation "A is at equilibrium with $\mathrm{B}$ " is transitive; if it holds for a pair of systems (A, B), and for another pair $(B, C)$, then it is true for $(A, C)$, too. Thus, for example, if we have sea ice forming a barrier between humid air and 
water and in thermodynamic equilibrium with each of them, then the air and the water are also in thermodynamic equilibrium even though they are not in contact; if the ice layer were removed, the remaining humid air and water would be in equilibrium. Either the seawater or the humid air may also be removed without upsetting the equilibrium between the remaining pair. Similarly, if a hole is made in the ice barrier, no adjustment is required to maintain equilibrium. Note that if ice were added to a system containing air and seawater at equilibrium, then establishing equilibrium conditions for the new system may require property adjustments in the air and seawater. However, if a new equilibrium is achieved containing all three elements then any one of them may be removed at constant $T$ and $P$ without upsetting the equilibrium between the remaining pair. In this sense, the properties of the equilibrium between ice and humid air, briefly "ice air", are independent of the presence or distribution of seawater; similarly, the equilibrium state between seawater and ice, "sea ice", does not depend on the continued presence of the ambient air components. Thermodynamic equations for sea ice were derived from the Gibbs functions of seawater and of ice by Feistel and Hagen (1998). Quantitative formulas for airfree sublimation pressure and enthalpy are available down to $50 \mathrm{~K}$ from Feistel and Wagner (2007) and IAPWS (2008b).

The three-phase system, referred to here as "sea-ice air", has the independent variables $T$ and $P$ as well as the mass fractions of salt, $w^{\mathrm{S}}$, and of air, $w^{\mathrm{A}}$, with respect to the system's total mass. The special cases of sublimation equilibria or of sea ice are available from the formulas considered here in the limits of vanishing sea salt, $w^{\mathrm{S}}=0$, or of air, $w^{\mathrm{A}}=0$, respectively. The salt-free equilibria are considered in more detail in Sect. 9. The relative spatial distribution of the three phases is not relevant here for the thermodynamic properties of the parcel as a whole, for instance, the humid air part may be completely separated from the seawater part by a surface layer of ice. This is true since the equilibrium between three phases always implies equilibrium conditions for each chosen pair of phases.

Of particular interest is the special case of ice air at zero salinity, $w^{\mathrm{S}}=0$, i.e. the properties of saturated air containing ice as in cirrus clouds or ice fog, air resting over a frozen lake or precipitating frost. The equations and formulas are similar to those for wet air, Sect. 7, except that the liquidwater properties of that section are substituted by the related ice properties for the application below the freezing point. The freezing point $T(P)$ of liquid water is computed from the equilibrium condition of equal chemical potentials of the two phases,

$g^{\mathrm{W}}(T, P)=g^{\mathrm{Ih}}(T, P)$.

Here, $g^{\mathrm{W}}$ is the Gibbs function of liquid water, Eq. (3.3), and $g^{\text {Ih }}$ is the Gibbs function of ice, Eq. (F1). At $101325 \mathrm{~Pa}$, the freezing temperature of pure water is $273.152519(2) \mathrm{K}$ (Feistel and Wagner, 2006; IAPWS, 2008b, 2009b), and is lowered by about $2.4 \mathrm{mK}$ if dissolved air is present (Doherty and Kester, 1974; Feistel, 2008a).

In this section, we generalize the Gibbs function of sea air, $g^{\text {SA }}$, Sect. 6 , to the Gibbs function of sea-ice air, $g^{\text {SIA }}$, extending the validity of Eq. (6.2) below the freezing temperature of water/seawater,

$$
\begin{gathered}
g^{\mathrm{SIA}}\left(w^{\mathrm{A}}, w^{\mathrm{S}}, T, P\right)=w^{\mathrm{AV}} g^{\mathrm{AV}}(A, T, P) \\
+w^{\mathrm{SW}} g^{\mathrm{SW}}\left(S_{\mathrm{A}}, T, P\right)+w^{\mathrm{Ih}} g^{\mathrm{Ih}}(T, P)
\end{gathered}
$$

The sample of sea-ice air has the total mass $m^{\mathrm{SIA}}=m^{\mathrm{SW}}+$ $m^{\mathrm{AV}}+m^{\mathrm{Ih}}$, consisting of the liquid phase, seawater, $m^{\mathrm{SW}}$, the gas phase, humid air, $m^{\mathrm{AV}}$, and the solid phase, ice $\mathrm{Ih}$, $m^{\text {Ih }}$. The mass fractions of seawater,

$w^{\mathrm{SW}}=m^{\mathrm{SW}} / m^{\mathrm{SIA}}=w^{\mathrm{S}} / S_{\mathrm{A}}$,

humid air,

$w^{\mathrm{AV}}=m^{\mathrm{AV}} / m^{\mathrm{SIA}}=w^{\mathrm{A}} / A$,

and ice, $w^{\text {Ih }}=m^{\text {Ih }} / m^{\text {SIA }}$, obey the identity

$w^{\mathrm{S}} / S_{\mathrm{A}}+w^{\mathrm{A}} / A+w^{\text {th }}=1$.

The equilibrium condition (6.4) of equal chemical potentials of water in the different phases takes the generalized form of the two separate equations

$$
\begin{aligned}
& \Lambda_{\mathrm{SI}}[g] \equiv g^{\mathrm{SW}}-S_{\mathrm{A}}\left(\frac{\partial g^{\mathrm{SW}}}{\partial S_{\mathrm{A}}}\right)_{T, P}-g^{\mathrm{Ih}}=0 \\
& \Lambda_{\mathrm{AI}}[g] \equiv g^{\mathrm{AV}}-A\left(\frac{\partial g^{\mathrm{AV}}}{\partial A}\right)_{T, P}-g^{\mathrm{Ih}}=0 .
\end{aligned}
$$

At given $T$ and $P$, these equations define the equilibrium fractions of air, $A(T, P)$, and salt, $S_{\mathrm{A}}(T, P)$, as well as the ice fraction, $w^{\mathrm{Ih}}\left(w^{\mathrm{S}}, w^{\mathrm{A}}, T, P\right)$, from Eq. (8.5). Between the freezing points of pure water and seawater the stable existence of freshwater (e.g., as fog droplets in the air) and of ice (e.g., as ice fog in the air) is impossible in sea air. The humid-air part, i.e. the gas fraction of dry air mixed with vapour, of sea air is always subsaturated, Fig. 10, as a result of the vapour-pressure lowering due to dissolved salt. In contrast, below the freezing point of seawater, ice is a stable phase (either within air or seawater) of sea-ice air and the humid-air fraction is always saturated since no relevant amounts of salt are "dissolved" in ice. Note that the freezing point of liquid water is not a distinguished temperature for vapour, regardless of whether or not air is present. As a stable gas phase of water, vapour can exist down to $40-50 \mathrm{~K}$ (Feistel and Wagner, 2007).

From the total derivative of Eq. (8.6), i.e. the ClausiusClapeyron differential equation for sea ice,

$$
\begin{aligned}
& \mathrm{d} \Lambda_{\mathrm{SI}}[g]=0=\left(\frac{\partial}{\partial S_{\mathrm{A}}} \Lambda_{\mathrm{SI}}[g]\right)_{T, P} \mathrm{~d} S_{\mathrm{A}} \\
& +\Lambda_{\mathrm{SI}}\left[\left(\frac{\partial g}{\partial T}\right)_{S_{\mathrm{A}}, P}\right] \mathrm{d} T+\Lambda_{\mathrm{SI}}\left[\left(\frac{\partial g}{\partial P}\right)_{S_{\mathrm{A}}, T}\right] \mathrm{d} P
\end{aligned}
$$



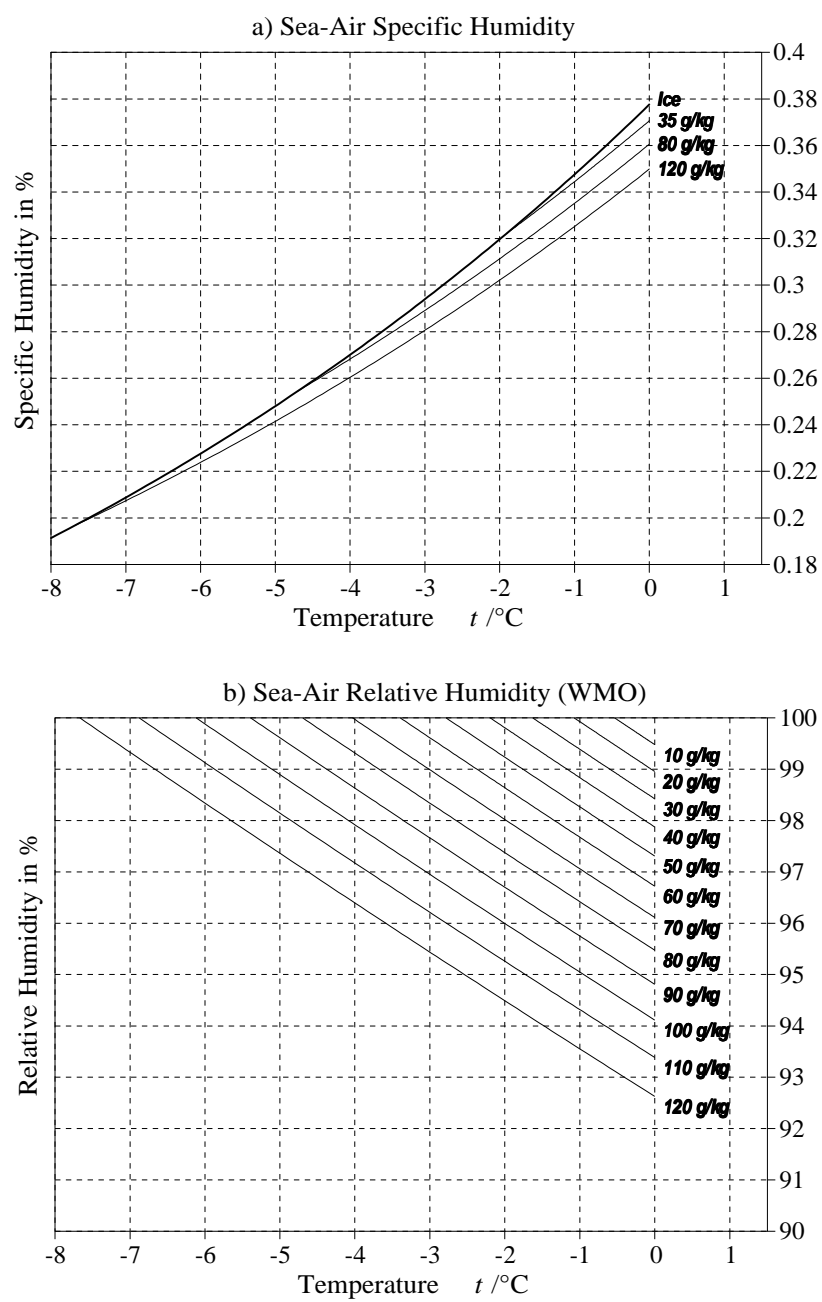

Fig. 10. Specific (a) and relative (b) humidity of sea air, i.e., humid air in equilibrium with seawater, at sea-level pressure, $P=101325 \mathrm{~Pa}$, as a function of temperature for different absolute salinities as indicated by the curves. The equilibrium air fraction $A$ is computed from Eq. (6.4); the resulting specific humidity is $q=(1-A)$. The relative humidity $R H_{\mathrm{WMO}}$ is determined from $A$ by the WMO definition (10.4) with the saturated air fraction $A^{\text {sat }}$ given by Eq. (8.7). The specific humidity related to $A^{\text {sat }}$ is displayed as the bold curve "Ice" a), indicating the saturation of sea-ice air due to ice formation below the freezing temperature at the particular salinity.

we infer the partial derivatives for the change of the brine salinity, $S_{\mathrm{A}}(T, P)$, independent of air,

$$
\left(\frac{\partial S_{\mathrm{A}}}{\partial T}\right)_{P}=-S_{\mathrm{A}} \frac{\Lambda_{\mathrm{SI}}[s]}{D_{\mathrm{S}}}, \quad\left(\frac{\partial S_{\mathrm{A}}}{\partial P}\right)_{T}=S_{\mathrm{A}} \frac{\Lambda_{\mathrm{SI}}[v]}{D_{\mathrm{S}}}
$$

where $D_{\mathrm{S}}$ is defined in Eq. (6.9).

For ice air we get from Eq. (8.7) the saturated value for the air fraction, $A=A_{\mathrm{AI}}^{\mathrm{sat}}(T, P)$, with respect to ice, independent of the presence of seawater. In the same way as in Eq. (8.8) we can derive the Clausius-Clapeyron differential equation of ice air from the differential of Eq. (8.7). The related isobaric and isothermal air drying rates are

$\delta_{P}^{\mathrm{AI}}=-\left(\frac{\partial A_{\mathrm{AI}}^{\mathrm{sat}}}{\partial T}\right)_{P}=A \frac{\Lambda_{\mathrm{AI}}[s]}{D_{\mathrm{A}}}$,

$\delta_{T}^{\mathrm{AI}}=-\left(\frac{\partial A_{\mathrm{AI}}^{\mathrm{sat}}}{\partial P}\right)_{T}=-A \frac{\Lambda_{\mathrm{AI}}[v]}{D_{\mathrm{A}}}$,

in which $D_{\mathrm{A}}$ is defined in Eq. (6.8). Crossing the freezing point $\left(T_{\mathrm{f}}, P_{\mathrm{f}}\right)$ of pure water, the drying rates of humid air change discontinuously from the ones relative to liquid water, Eqs. (7.7) and (7.8), to those relative to ice, Eq. (8.10), while the saturation value itself is continuous, i.e., $A_{\mathrm{AI}}^{\text {sat }}\left(T_{\mathrm{f}}, P_{\mathrm{f}}\right)=$ $A_{\mathrm{AW}}^{\mathrm{sat}}\left(T_{\mathrm{f}}, P_{\mathrm{f}}\right)$ at this point.

We infer from Eqs. (8.5), (8.9) and (8.10), that upon heating the ice fraction shrinks at the isobaric melting rate,

$\varepsilon_{P}^{\mathrm{SIA}}=-\left(\frac{\partial w^{\mathrm{Ih}}}{\partial T}\right)_{w^{\mathrm{A}}, w^{\mathrm{S}}, P}=w^{\mathrm{SW}} \frac{\Lambda_{\mathrm{SI}}[s]}{D_{\mathrm{S}}}+w^{\mathrm{AV}} \frac{\Lambda_{\mathrm{AI}}[s]}{D_{\mathrm{A}}}$,

or, when compressed, at the isothermal melting rate,

$\varepsilon_{T}^{\mathrm{SIA}}=-\left(\frac{\partial w^{\mathrm{Ih}}}{\partial P}\right)_{w^{\mathrm{A}}, w^{\mathrm{S}}, T}=-w^{\mathrm{SW}} \frac{\Lambda_{\mathrm{SI}}[v]}{D_{\mathrm{S}}}-w^{\mathrm{AV}} \frac{\Lambda_{\mathrm{AI}}[v]}{D_{\mathrm{A}}}$.

With the help of Eqs. (8.5-8.12) we can now compute the first and second derivatives of the Gibbs function (8.2) for a system composed of seawater, ice, and humid air.

The specific entropy of sea ice air, $s^{\text {SIA }}$, follows as

$s^{\mathrm{SIA}}=-\left(\frac{\partial g^{\mathrm{SIA}}}{\partial T}\right)_{w^{\mathrm{A}}, w^{\mathrm{S}}, P}=w^{\mathrm{AV}} s^{\mathrm{AV}}+w^{\mathrm{SW}} s^{\mathrm{SW}}+w^{\mathrm{Ih}} s^{\mathrm{hh}}$,

the enthalpy is

$h^{\mathrm{SIA}}=g^{\mathrm{SIA}}+T s^{\mathrm{SIA}}=w^{\mathrm{AV}} h^{\mathrm{AV}}+w^{\mathrm{SW}} h^{\mathrm{SW}}+w^{\mathrm{Ih}} h^{\mathrm{Ih}}$,

and the specific volume

$v^{\mathrm{SIA}}=\left(\frac{\partial g^{\mathrm{SIA}}}{\partial P}\right)_{w^{\mathrm{A}}, w^{\mathrm{S}}, T}=w^{\mathrm{AV}} v^{\mathrm{AV}}+w^{\mathrm{SW}} v^{\mathrm{SW}}+w^{\mathrm{Ih}} v^{\mathrm{Ih}}$.

Thus, the first derivatives of the Gibbs function are strictly additive with regard to the phases present.

For the second temperature derivative of $g^{\text {SIA }}$, exploiting Eqs. (8.13), (8.3), (8.4) we have

$$
\begin{aligned}
& \frac{c_{p}^{\mathrm{SIA}}}{T}=\left(\frac{\partial s^{\mathrm{SIA}}}{\partial T}\right)_{w^{\mathrm{A}}, w^{\mathrm{S}}, P}=w^{\mathrm{AV}}\left[\left(\frac{\partial s^{\mathrm{AV}}}{\partial T}\right)_{A, P}\right. \\
& \left.+\left(\frac{\partial s^{\mathrm{AV}}}{\partial A}\right)_{T, P}\left(\frac{\partial A}{\partial T}\right)_{w^{\mathrm{A}}, w^{\mathrm{S}}, P}\right]+w^{\mathrm{SW}}\left[\left(\frac{\partial s^{\mathrm{SW}}}{\partial T}\right)_{S_{\mathrm{A}}, P}\right. \\
& \left.+\left(\frac{\partial s^{\mathrm{SW}}}{\partial S_{\mathrm{A}}}\right)_{T, P}\left(\frac{\partial S_{\mathrm{A}}}{\partial T}\right)_{w^{\mathrm{A}}, w^{\mathrm{S}}, P}\right]+w^{\mathrm{Ih}}\left(\frac{\partial s^{\mathrm{Ih}}}{\partial T}\right)_{P} \\
& -s^{\mathrm{AV}} \frac{w^{\mathrm{A}}}{A^{2}}\left(\frac{\partial A}{\partial T}\right)_{w^{\mathrm{A}}, w^{\mathrm{S}}, P}-s^{\mathrm{SW}} \frac{w^{\mathrm{S}}}{S_{\mathrm{A}}^{2}}\left(\frac{\partial S_{\mathrm{A}}}{\partial T}\right)_{w^{\mathrm{A}}, w^{\mathrm{S}}, P} \\
& +s^{\mathrm{Ih}}\left(\frac{\partial w^{\mathrm{Ih}}}{\partial T}\right)_{w^{\mathrm{A}}, w^{\mathrm{S}}, P}
\end{aligned}
$$


from which we obtain the formula for the isobaric heat capacity,

$$
\begin{aligned}
& c_{p}^{\mathrm{SIA}}=w^{\mathrm{AV}} c_{p}^{\mathrm{AV}}+w^{\mathrm{SW}} c_{p}^{\mathrm{SW}}+w^{\mathrm{Ih}} c_{p}^{\mathrm{Ih}} \\
& +T\left\{w^{\mathrm{AV}} \frac{\left(\Lambda_{\mathrm{AI}}[s]\right)^{2}}{D_{\mathrm{A}}}+w^{\mathrm{SW}} \frac{\left(\Lambda_{\mathrm{SI}}[s]\right)^{2}}{D_{\mathrm{S}}}\right\} .
\end{aligned}
$$

In the scientific literature, we are not aware of a thermodynamically rigorous definition of the latent heat that occurs in conjunction with the exchange of water between seawater, humid air and ice. We define here the latent-heat part of the heat capacity (8.17), $T\left\{w^{\mathrm{AV}}\left(\Lambda_{\mathrm{AI}}[s]\right)^{2} / D_{\mathrm{A}}+w^{\mathrm{SW}}\left(\Lambda_{\mathrm{SI}}[s]\right)^{2} D_{\mathrm{S}}\right\}$, divided by the isobaric melting rate, i.e. the mass fraction loss of ice, $-\left(\partial w^{\mathrm{Ih}} / \partial T\right)_{w^{\mathrm{A}}, w^{\mathrm{s}}, P}$ from Eq. (8.11), as the isobaric latent heat of ice, $L_{P}^{\text {SIA }}$, of this system,

$L_{P}^{\mathrm{SIA}}=\frac{w^{\mathrm{AV}}\left(\Lambda_{\mathrm{AI}}[h]\right)^{2} / D_{\mathrm{A}}+w^{\mathrm{SW}}\left(\Lambda_{\mathrm{SI}}[h]\right)^{2} / D_{\mathrm{S}}}{w^{\mathrm{AV}} \Lambda_{\mathrm{AI}}[h] / D_{\mathrm{A}}+w^{\mathrm{SW}} \Lambda_{\mathrm{SI}}[h] / D_{\mathrm{S}}}$.

This expression includes both the latent heats of melting (subscript SI) and of sublimation (AI), and only indirectly that of evaporation (AS). Rather than simply being added up, the transition enthalpies appear averaged, weighted by sublimation and melting rates. For freshwater $\left(w^{\mathrm{S}}=0\right)$, this formula for $L_{P}^{\mathrm{SIA}}$ reduces to the sublimation enthalpy in air, $\Lambda_{\mathrm{AI}}[h]$,

$L_{P}^{\mathrm{AI}}=\Lambda_{\mathrm{AI}}[h] \equiv h^{\mathrm{AV}}-A\left(\frac{\partial h^{\mathrm{AV}}}{\partial A}\right)_{T, P}-h^{\mathrm{Ih}}$.

Alternatively, for air-free conditions $\left(w^{\mathrm{A}}=0\right)$, it provides the melting enthalpy in seawater, $\Lambda_{\mathrm{SI}}[h]$

$L_{P}^{\mathrm{SI}}=\Lambda_{\mathrm{SI}}[h] \equiv h^{\mathrm{SW}}-S_{\mathrm{A}}\left(\frac{\partial h^{\mathrm{SW}}}{\partial S_{\mathrm{A}}}\right)_{T, P}-h^{\mathrm{Ih}}$.

The expressions for the latent heat must be independent of the freely adjustable coefficients. It is easily verified that Eqs. (8.18-8.20) satisfy this necessary condition. In all cases, using the simple difference between the enthalpies of ice and seawater or humid air rather than Eqs. (8.19), (8.20), would be essentially wrong for this reason. In other words, the numerical values of the additional saline enthalpy term $S_{\mathrm{A}} \times\left(\partial h^{\mathrm{SW}} / \partial S_{\mathrm{A}}\right)_{T, P}$ or the humid-air enthalpy term $A \times\left(\partial h^{\mathrm{AV}} / \partial A\right)_{T, P}$ depend on the arbitrary choices made for the adjustable coefficients and must not be neglected.

When changes in $T$ or $P$ cause a certain water mass to be transferred between ice and the two fluid phases, there is no additional direct transfer of water between those fluids, seawater and humid air, i.e., Eq. (8.18) covers the latent heat of all three phases below the freezing point. This means that Eq. (8.18) can also be expressed in terms of the transition properties of the other two possible phase pairs, sublimation (AI) and evaporation (SA), or melting (SI) and evaporation (SA), using the relation (6.6), $\Lambda_{\mathrm{SA}}=\Lambda_{\mathrm{SI}}-\Lambda_{\mathrm{AI}}$.

\section{Properties of ice air}

In the limit of zero salinity, the composite system "sea ice air" described in the previous section turns into "wet ice air", i.e. the equilibrium of liquid water, ice and vapour in the presence of air. When there is no air, $w^{\mathrm{A}}=0$, temperature and pressure of this system equal the triple-point temperature and pressure of water, while the mutual mass ratios of the three phases are controlled by two additional constraints such as the volume and the total entropy of the sample. When air is added, the partial vapour pressure $P^{\mathrm{V}}$ will remain close to the triple-point pressure but the total pressure, $P=P^{\mathrm{V}} / x_{\mathrm{V}}^{\mathrm{AV}}$, Eq. (5.6), of such an air parcel will be different, Fig. 11. This state is controlled by Eqs. (8.5-8.7) at zero salinity, i.e.,

$\Lambda_{\mathrm{WI}}[g] \equiv g^{\mathrm{W}}-g^{\mathrm{Ih}}=0$

$\Lambda_{\mathrm{AI}}[g] \equiv g^{\mathrm{AV}}-A\left(\frac{\partial g^{\mathrm{AV}}}{\partial A}\right)_{T, P}-g^{\mathrm{Ih}}=0$.

This system of equations can be solved for the temperature $T(A)$ and the pressure $P(A)$ as functions of the air fraction A.

The three mass fractions of the parcel's phases, liquid water, $w^{\mathrm{W}}$, ice, $w^{\mathrm{Ih}}$, and saturated humid air, $w^{\mathrm{AV}}=w^{\mathrm{A}} / A$, are related to temperature and pressure via the mass fraction $A(T, P)$ of dry air in humid air, i.e., to the solution of Eqs. (9.1), (9.2):

$w^{\mathrm{W}}+w^{\mathrm{Ih}}+\frac{w^{\mathrm{A}}}{A}=1$.

At a given air fraction $w^{\mathrm{A}}$ and entropy $s$, Fig. 12,

$s=w^{\mathrm{W}} s^{\mathrm{W}}(T, P)+w^{\mathrm{Ih}} s^{\mathrm{Ih}}(T, P)+\frac{w^{\mathrm{A}}}{A} s^{\mathrm{AV}}(A, T, P)$

this system of wet ice air still possesses one degree of freedom. We term the pressure it takes at complete melting, $w^{\mathrm{Ih}}=0$, the isentropic freezing level (IFL) of wet air, and at complete freezing, $w^{\mathrm{W}}=0$, the isentropic melting level (IML) of ice air. For sea air with entropy and air fraction computed from Eqs. (6.4) and (6.18), these curves are displayed in Fig. 17.

At pressures below the IML, the liquid phase has disappeared and the system reduces to "ice air".

\section{Relative humidity and fugacity}

Relative humidity is not uniquely defined in the literature. Sometimes it is defined as the ratio of the actual partial pressure of vapour in air to the saturated vapour pressure, and sometimes as the ratio of their corresponding mole fractions (Van Wylen and Sonntag, 1965; Sonntag, 1982). All definitions give the same results in the ideal-gas limit. Also in 


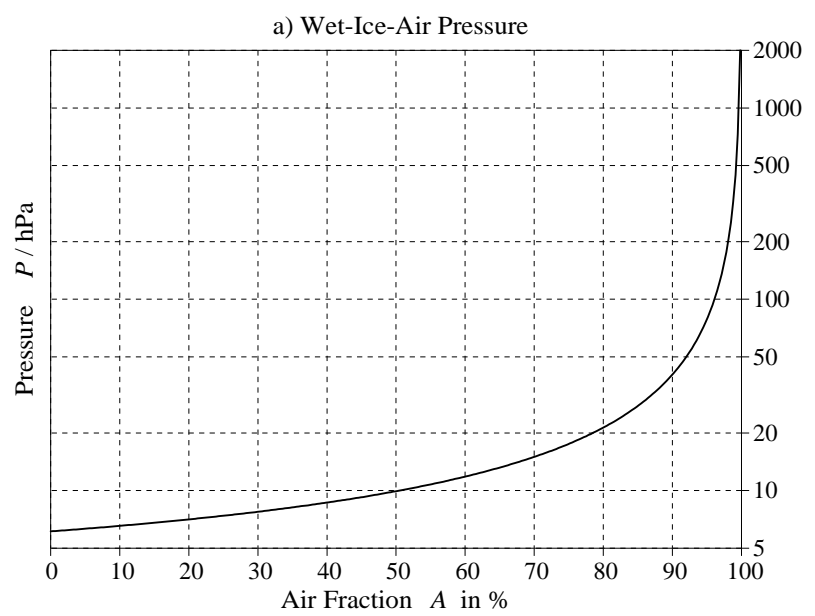

b) Wet-Ice-Air Temperature

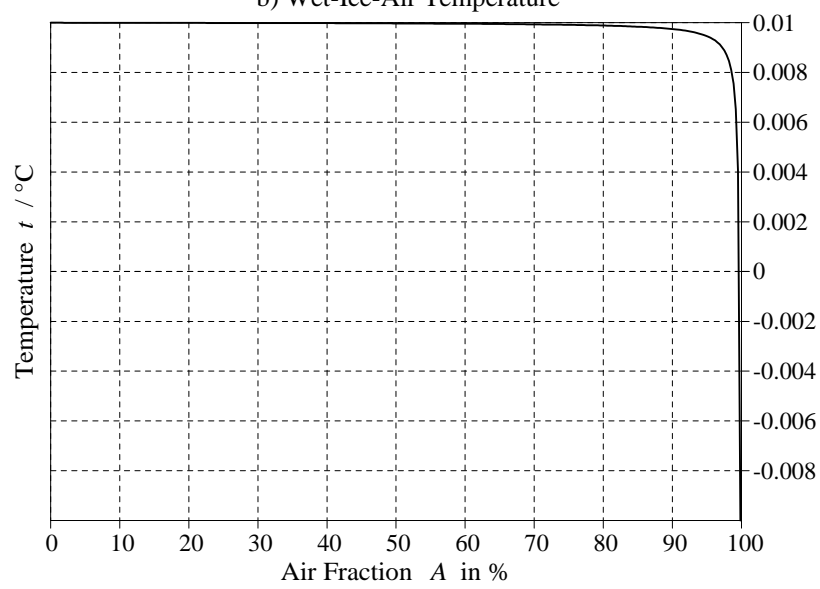

Fig. 11. Equilibrium pressure $P$, panel (a), and temperature $T$, panel (b), as a function of the mass fraction $A$ of dry air in humid air of a parcel containing liquid water, ice and humid air. At $A=0$, the curve ends in the common triple point of water at about $611 \mathrm{~Pa}$ and $273.16 \mathrm{~K}$. Note that the dissolution of air in liquid water is not considered here which may have comparable additional effects on the freezing temperature (Doherty and Kester, 1974; IAPWS, 2004; Feistel, 2008a).

this approximation, relative humidity is a property of fluid water at given temperature and pressure of the vapour phase, independent of the presence of air.

The $\mathrm{CCT}^{10}$ definition of relative humidity is in terms of mole fraction: at given pressure and temperature, the ratio, expressed as a percent, of the mole fraction of water vapour to the vapour mole fraction which the moist gas would have if it were saturated with respect to either water or ice at the same pressure and temperature. Consistent with CCT, IUPAC $^{11}$ defines relative humidity as the ratio, often expressed as a percentage, of the partial pressure of water in the at-

\footnotetext{
${ }^{10}$ CCT: Consultative Committee for Thermometry, www.bipm. org/en/committees/cc/cct/.

${ }^{11}$ IUPAC: International Union of Pure and Applied Chemistry, www.iupac.org.
}
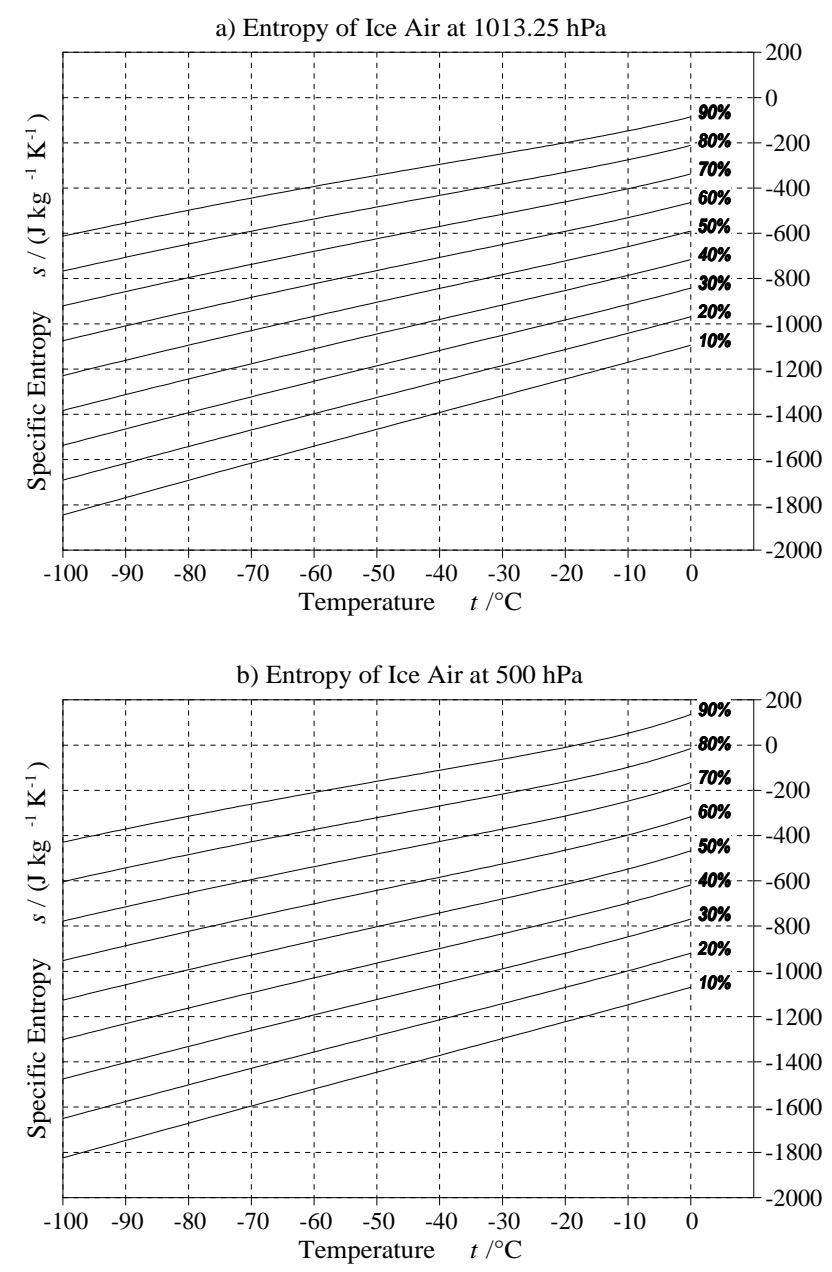

Fig. 12. Entropy of ice air as a function of temperature at $1013.25 \mathrm{hPa}$ (a) and $500 \mathrm{hPa}$ (b) between $-100^{\circ} \mathrm{C}$ and the melting point of the solid component for dry-air fractions $w^{\mathrm{A}}$ of ice air between $10 \%$ and $90 \%$ as indicated by the curves.

mosphere at some observed temperature, to the saturation vapour pressure of pure water at this temperature (Calvert, 1990; IUPAC, 1997). For a real-gas mixture such as air, partial pressures of its components are defined by the total pressure multiplied by the mole fraction of that component (Lehmann et al., 1996; IUPAC, 1997)

$P^{a}=x_{a} P$.

This definition of the relative humidity takes the form

$R H_{\mathrm{CCT}}=\frac{x_{\mathrm{V}}^{\mathrm{AV}}}{x_{\mathrm{V}}^{\mathrm{AV}, \mathrm{sat}}}$

with regard to the mole fraction of vapour $x_{\mathrm{V}}^{\mathrm{AV}}(A)$ from Eq. (5.6) and the saturated air fraction $A^{\text {sat }}(T, P)$ from Eq. (7.5) with respect to liquid water and from Eq. (8.7) with respect to ice. The pressure derivative provides the rate of relative humidification upon adiabatic uplift, 


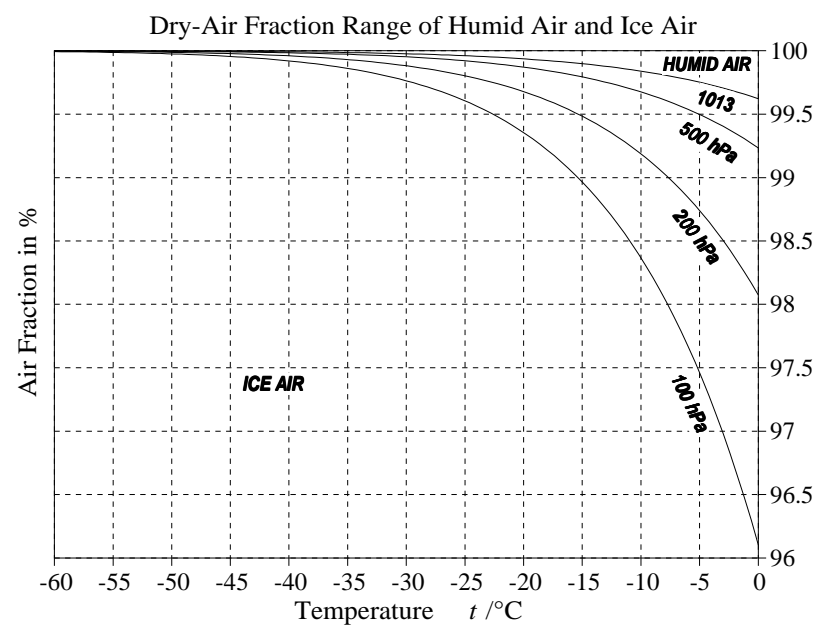

Fig. 13. Saturation curves $A_{\mathrm{AI}}^{\mathrm{sat}}(T, P)$ computed by solving Eq. (9.2) at the pressures $1013.25,500,200$ and $100 \mathrm{hPa}$, as indicated. Between $-60^{\circ} \mathrm{C}$ and the melting temperature of ice, these curves separate the physically reasonable dry-air fractions, $A \geq A_{\mathrm{AI}}^{\mathrm{sat}}(T, P)$, of the single-phase state of humid air from those of the two-phase state of ice air, $w^{\mathrm{A}} \leq A_{\mathrm{AI}}^{\mathrm{sat}}(T, P)$. At the saturation point, the two systems coincide.

$$
\begin{aligned}
& \chi(A, T, P)=-\left(\frac{\partial R H_{\mathrm{CCT}}}{\partial P}\right)_{A, s} \\
& =\frac{x_{\mathrm{V}}^{\mathrm{AV}} M^{\mathrm{W}}}{M^{\mathrm{A}}\left(1-A^{\mathrm{sat}}\right)^{2}}\left(\delta_{T}^{\mathrm{AW}}+\Gamma^{\mathrm{AV}} \delta_{P}^{\mathrm{AW}}\right) .
\end{aligned}
$$

Here, the drying rates $\delta^{\mathrm{AW}}$ with respect to liquid water and the lapse rate $\Gamma^{\mathrm{AV}}$ of humid air are defined in Eqs. (7.7), (7.8) and (5.25). Below the freezing point, $\delta^{\mathrm{AW}}$ must be replaced by $\delta^{\mathrm{AI}}$ with respect to ice, Eq. (8.10).

The $\mathrm{WMO}^{12}$ definition of the relative humidity is (Rogers and Yau, 1989; Pruppacher and Klett, 1997; Jacobson, 2005),

$R H_{\mathrm{WMO}}=\frac{r}{r^{\mathrm{sat}}}=\frac{1 / A-1}{1 / A^{\mathrm{sat}}-1}$

where $r=(1-A) / A=m^{\mathrm{V}} / m^{\mathrm{A}}$ is the humidity ratio. The relation (10.4) between air fraction, temperature and relative humidity is shown quantitatively in Fig. 14.

If $r$ is small, we can estimate $x_{\mathrm{V}}^{\mathrm{AV}} \approx r M^{\mathrm{A}} / M^{\mathrm{W}}$ from Eq. (5.6) and therefore $R H_{\mathrm{WMO}} \approx R H_{\mathrm{CCT}}$, i.e. approximate consistency of Eqs. (10.2) and (10.4).

The adiabatic humidification rate, i.e. the rate of increase of relative humidity of an adiabatically lifted air parcel follows from Eqs. (10.4) and (7.9) as

\footnotetext{
${ }^{12}$ WMO: World Meteorological Organisation, www.wmo.int.
}

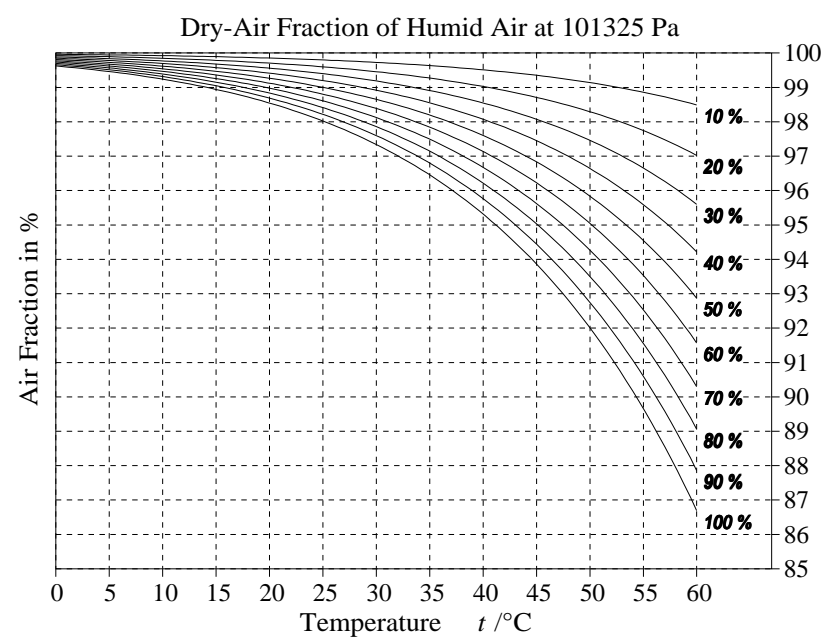

Fig. 14. Mass fraction $A$ of dry air in humid air as a function of temperature at nomal pressure, $101325 \mathrm{~Pa}$, for different relative humidities (WMO definition, Eq. 10.4) as indicated by the curves from $10 \%$ to $100 \%$ (dewpoint). Specific humidity is $q=1-A$. Below the dewpoint curve $(100 \%)$, liquid water is present, i.e., the stable state is wet air rather than humid air.

$$
\begin{gathered}
\chi(A, T, P)=-\left(\frac{\partial R H_{\mathrm{WMO}}}{\partial P}\right)_{A, s} \\
=\frac{r}{\left(1-A_{\mathrm{AW}}^{\mathrm{sat}}\right)^{2}}\left(\delta_{T}^{\mathrm{AW}}+\Gamma^{\mathrm{AV}} \delta_{P}^{\mathrm{AW}}\right) .
\end{gathered}
$$

Sometimes, especially when considering phase or chemical equilibria, it is more convenient to use the fugacity (or activity) rather than partial pressure (Lewis, 1908; Glasstone, 1947; Möbius and Dürselen, 1973; Ewing et al., 1994; IUPAC, 1997; Blandamer et al., 2005). The fugacity of vapour in humid air is defined as, Eq. (5.31),

$$
f_{\mathrm{V}}(A, T, P)=f_{\mathrm{V}}^{0}(T) \exp \left\{\frac{\mu^{\mathrm{V}}(A, T, P)}{R_{\mathrm{W}} T}\right\} .
$$

The chemical potential of vapour in humid air, $\mu^{\mathrm{V}}$, is given in Eq. (5.15). $R_{\mathrm{W}}$ is the specific gas constant of water. The reference fugacity,

$$
\begin{aligned}
& f_{\mathrm{V}}^{0}(T)=\lim _{P \rightarrow 0} f_{\mathrm{V}}(A, T, P) \exp \left\{-\frac{\mu^{\mathrm{V}}}{R_{\mathrm{W}} T}\right\} \\
& =x_{\mathrm{V}} P \exp \left\{-\frac{\mu^{\mathrm{V}, \mathrm{id}}}{R_{\mathrm{W}} T}\right\}
\end{aligned}
$$

is chosen such that in the ideal-gas limit of infinite dilution, $f_{\mathrm{V}}$ converges to the partial pressure of vapour (Glasstone, 1947; Guggenheim, 1967), Eq. (10.1),

$f_{\mathrm{V}}=x_{\mathrm{V}}^{\mathrm{AV}} P \exp \left\{\frac{\mu^{\mathrm{V}}-\mu^{\mathrm{V}, \mathrm{id}}}{R_{\mathrm{W}} T}\right\}$. 
The ideal-gas chemical potential $\mu^{\mathrm{V} \text {,id }}$ is given in Eq. (H13), and the mole fraction of vapour in humid air, $x_{\mathrm{V}}^{\mathrm{AV}}$, is given in Eqs. (5.6) and (B9).

The ratio $\lambda_{\mathrm{V}}=f_{\mathrm{V}} / f_{\mathrm{V}}^{0}$ is the absolute activity of vapour (Ewing et al., 1994; IUPAC, 1997).

The fugacity-pressure ratio $f_{\mathrm{V}} /\left(x_{\mathrm{V}}^{\mathrm{AV}} P\right)$ is known as the fugacity coefficient or enhancement factor in meteorology.

The saturation fugacity is defined by the equilibrium between liquid water and vapour in air, $\mu^{\mathrm{V}}(A, T, P)=$ $\mu^{\mathrm{W}}(0, T, P)$, i.e.

$f_{\mathrm{V}}^{\mathrm{sat}}=f_{\mathrm{V}}^{0}(T) \exp \left\{\frac{\mu^{\mathrm{W}}(0, T, P)}{R_{\mathrm{W}} T}\right\}$,

where $\mu^{\mathrm{W}}=g^{\mathrm{W}}(T, P)=g^{\mathrm{SW}}(0, T, P)$ is the chemical potential of liquid water, i.e. its Gibbs energy (3.3). The relative fugacity $\varphi$ of humid air can thus be defined as

$\varphi=\frac{f_{\mathrm{V}}}{f_{\mathrm{V}}^{\mathrm{sat}}}=\exp \left\{\frac{\mu^{\mathrm{V}}(A, T, P)-\mu^{\mathrm{W}}(0, T, P)}{R_{\mathrm{W}} T}\right\}$.

In the ideal-gas limit, $\mu^{\mathrm{V}}=\mu^{\mathrm{V} \text {,id }}$, the relative fugacity $\varphi$ approaches the relative humidity (10.2) because of Eq. (10.8).

It follows that the relative fugacity of sea air is

$\varphi^{\mathrm{SA}}=\frac{f_{\mathrm{V}}^{\mathrm{SA}}}{f_{\mathrm{V}}^{\mathrm{sat}}}=\exp \left\{\frac{\mu^{\mathrm{W}}\left(S_{\mathrm{A}}, T, P\right)-\mu^{\mathrm{W}}(0, T, P)}{R_{\mathrm{W}} T}\right\}$

since its vapour is assumed to be in equilibrium (6.4) with seawater, $\mu^{\mathrm{V}}(A, T, P)=\mu^{\mathrm{W}}\left(S_{\mathrm{A}}, T, P\right)$.

The chemical potential difference in the exponent is proportional to the osmotic coefficient of seawater, $\phi^{\mathrm{SW}}$, which is computed from the saline part of the Gibbs function, Eq. (6.1), as (Feistel and Marion, 2007; Feistel, 2008a),

$\phi^{\mathrm{SW}}\left(S_{\mathrm{A}}, T, P\right)=-\frac{1}{m_{\mathrm{SW}} R T}\left[g^{\mathrm{S}}-S_{\mathrm{A}}\left(\frac{\partial g^{\mathrm{S}}}{\partial S_{\mathrm{A}}}\right)_{T, P}\right]$,

and $m_{\mathrm{SW}}$ is the molality of seawater (Millero et al., 2008),

$m_{\mathrm{SW}}=\frac{S_{\mathrm{A}}}{\left(1-S_{\mathrm{A}}\right) M^{\mathrm{S}}}$.

We infer for the relative fugacity of sea air the simple formula

$\varphi^{\mathrm{SA}}=\exp \left(-m_{\mathrm{SW}} M^{\mathrm{W}} \phi^{\mathrm{SW}}\right)$,

which is also known as the activity $a_{\mathrm{W}}$ of water in seawater (Falkenhagen et al., 1971; Millero and Leung, 1976; Feistel and Marion, 2007). Similar to the ideal gas approximation, the relative fugacity of sea air is independent of the presence or the properties of air. In Eq. (10.14), $\varphi^{\mathrm{SA}} \leq 1$ expresses the fact that the vapour pressure of seawater is lower than the saturation pressure of liquid pure water, i.e., that humid air in equilibrium with seawater above its freezing temperature is always subsaturated.
In first approximation, derived from a series expansion with respect to salinity, we obtain from Eq. (10.9), $m_{\mathrm{SW}}=$ $S_{\mathrm{A}} / M^{\mathrm{S}}+O\left(S_{\mathrm{A}}^{2}\right)$, and $\phi^{\mathrm{SW}}=1+O\left(S_{\mathrm{A}}\right)$ the linear relation

$\varphi^{\mathrm{SA}} \approx 1-\frac{M^{\mathrm{W}}}{M^{\mathrm{S}}} S_{\mathrm{A}}$

from Eq. (10.14), i.e. Raoult's law for the vapour-pressure lowering of seawater (Landau and Lifschitz, 1987; Feistel et al., 2008b). In the ideal-gas limit, Eqs. (10.14) and (10.15) are valid, too, for the relative humidity, $\varphi^{\mathrm{SA}} \approx R H$.

Below the freezing temperature of pure water at given pressure, the saturated vapour is defined by the chemical potential of ice rather than liquid water, i.e. by

$f_{\mathrm{V}}^{\mathrm{sat}}=f_{\mathrm{V}}^{0}(T) \exp \left\{\frac{\mu^{\mathrm{Ih}}(T, P)}{R_{\mathrm{W}} T}\right\}$,

instead of Eq. (10.9). Then, the relative fugacity of sea air is

$\varphi^{\mathrm{SA}}=\frac{f_{\mathrm{V}}^{\mathrm{SA}}}{f_{\mathrm{V}}^{\mathrm{sat}}}=\exp \left\{\frac{\mu^{\mathrm{W}}\left(S_{\mathrm{A}}, T, P\right)-\mu^{\mathrm{Ih}}(T, P)}{R_{\mathrm{W}} T}\right\}$

When the temperature is lowered further to the freezing point of seawater, the exponent of Eq. (10.17) vanishes and the air is saturated, $\phi^{\mathrm{SA}}=1$, for sea-ice air at any lower temperature.

\section{Isentropic expansion of sea-air}

In an external gravity field, equilibrium states possess vertically constant temperature and chemical potentials but are stratified in entropy and concentrations (Landau and Lifschitz, 1987). Adiabatically mixed air columns are better approximations of the real atmosphere, characterized by the conjugate properties of constant entropy and concentrations with temperature and chemical potentials depending on the vertical coordinate (Feistel and Feistel, 2006). It must be emphasised that an adiabatically well-mixed air column in a gravity field is not in thermodynamic equilibrium since the irreversible thermodynamic driving forces are the nonzero gradients of in-situ temperature and chemical potentials, rather than those of potential temperature or concentrations (Glansdorff and Prigogine, 1971). Due to the sluggish molecular relaxation in comparison to turbulent mixing, equilibrium states are prevented from being approached under ambient conditions. While the air column as a whole therefore remains far from overall equilibrium, each thin isobaric layer is assumed in the following to be in local thermodynamic equilibrium. In particular thermodynamic equilibrium is assumed to hold within a boundary layer extending only slightly above and below the sea surface.

In the sea-air atmosphere model of this section we consider an air column which is in equilibrium with the sea surface and "vertically" isentropic. The pressure is used here 
as the "vertical" coordinate so that gravity is only implicitly considered. The vertical profile of the real atmosphere is simulated by isentropic expansion of a parcel with given mass fractions of air and water. All thermodynamic properties of the vertical profile of the sea-air atmosphere model can be computed from three given independent variables, the in-situ temperature at the surface (sea-surface temperature, SST), the sea-level pressure (SLP), here assumed as $101325 \mathrm{~Pa}$, and the sea-surface salinity (SSS), here assumed as $35.16504 \mathrm{~g} / \mathrm{kg}$ (Millero et al., 2008).

Depending on pressure and entropy as its natural variables, enthalpy $h$ is the most convenient thermodynamic potential function for the description of adiabatic processes (Feistel and Hagen, 1995; Feistel, 2008a). The "vertical" isentropic density profile is computed from

$\rho(P)=\left(\frac{\partial h}{\partial P}\right)_{w^{\mathrm{A}}, s}^{-1}$

and the isentropic temperature profile (Fig. 16) from

$T(P)=\left(\frac{\partial h}{\partial s}\right)_{w^{\mathrm{A}}, P}$.

Here, $w^{\mathrm{A}}$ is the mass fraction of dry air, $h$ the enthalpy and $s$ the entropy of either humid air or of the composite systems wet air, ice air, or wet ice air. Solving Eqs. (7.25), (7.26), the subsaturated equilibrium humidity of sea air (Fig. 3) results in an isentropic condensation level (ICL) of 4-5 hPa below surface pressure (Figs. 15, 16). In Earth's atmosphere, this corresponds to an altitude of about $30-40 \mathrm{~m}$ above the sea surface.

Below the dewpoint temperature, the sea-air potential must be replaced by the wet-air potential (7.1), and below the frost point it is replaced by the corresponding iceair potential. Note that in doing this, we are considering condensed/frozen water as a floating, not precipitating aerosol/fog/cloud. Recall that any fallout from the sample is neglected by the model approach of an isentropically expanded, gravity-free parcel.

The adiabatic lapse rate of sea air at the surface is available from the formula

$\Gamma=\left(\frac{\partial T}{\partial P}\right)_{w^{\mathrm{A}}, w^{\mathrm{s}}, s}=\left(\frac{\partial^{2} h^{\mathrm{SA}}}{\partial s \partial P}\right)_{w^{\mathrm{A}}, w^{\mathrm{s}}}$.

\section{Discussion}

The isentropic expansion of sea air considered in the previous section represents a rather idealized model of the marine atmosphere. In this section, we consider for illustration purposes some examples taken from observations and compare them with related properties of the sea-air model. Frequently, the ambient atmosphere is not in an equilibrium state with

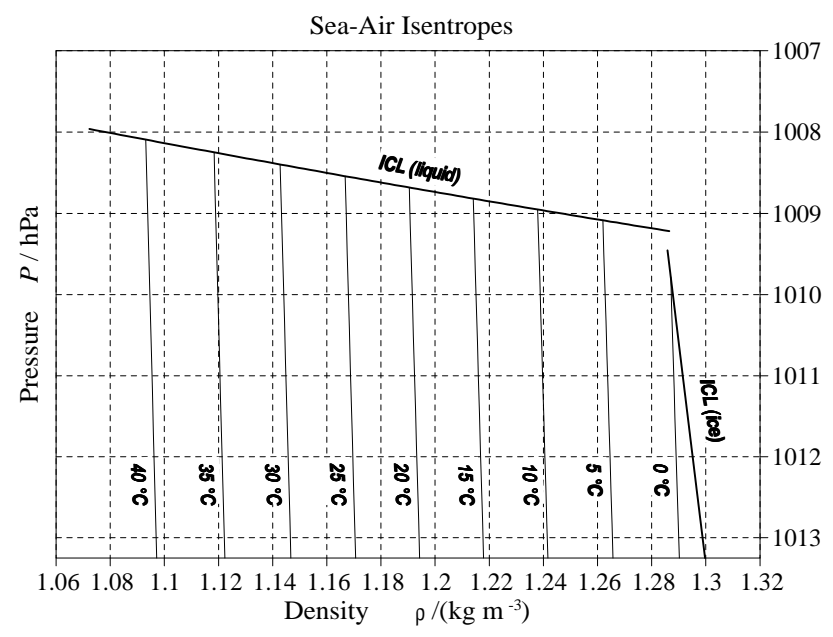

Fig. 15. Vertical isentropic sea-air profiles of density overlying seawater at standard-ocean salinity $\left(35.16504 \mathrm{~g} \mathrm{~kg}^{-1}\right)$ and sea-level pressure $(101325 \mathrm{~Pa})$ for different SST values between 0 and $40^{\circ} \mathrm{C}$ as indicated. The related isentropic condensation levels (ICL) of liquid water and ice (bold lines) are also shown.

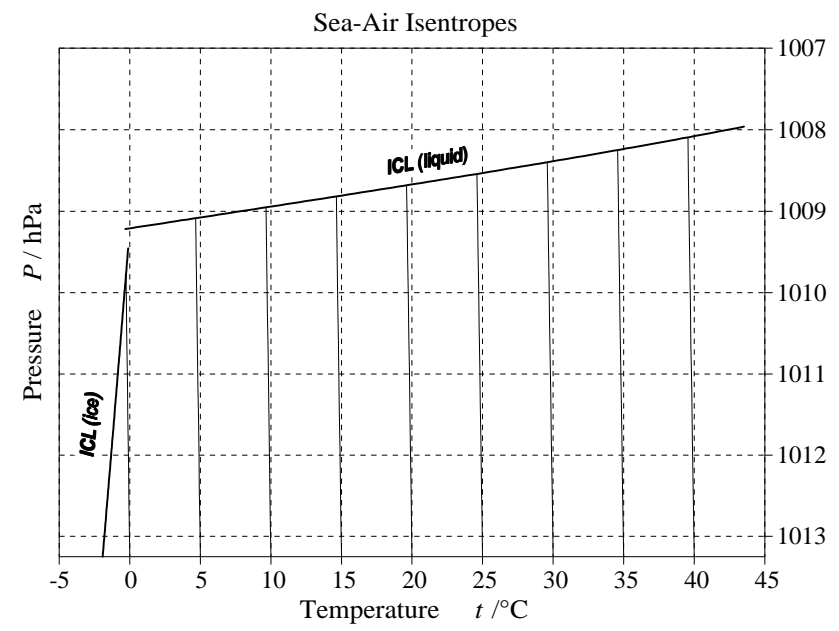

Fig. 16. Vertical isentropic sea-air profiles (almost vertical thin lines) of temperature for different SST values between 0 and $40^{\circ} \mathrm{C}$ at standard-ocean salinity and sea-level pressure of $101325 \mathrm{~Pa}$, together with the related isentropic condensation levels (ICL) of liquid water and ice (bold lines). Below the freezing temperature of seawater, sea air is saturated at the sea surface. The slope of the isentropes is given by the adiabatic lapse rate, Eqs. (6.25), (6.26).

the liquid or solid water phase, neither at the sea surface nor within clouds (Korolev and Isaac, 2006). Thermodynamics can be used to estimate the distance from equilibrium and the related irreversible fluxes.

During the oceanographic expedition AMOR-92 of $\mathrm{r} / \mathrm{v}$ "A. v. Humboldt" in the late summer of 1992 in the Atlantic coastal upwelling region off Morocco (Hagen et al., 1996), regular radiosonde sampling of the atmosphere was carried out. To provide comparisons with examples of real maritime 
atmospheric profiles, we have selected here two casts as described to Table 1 and shown in Fig. 19. In RS \#37 we see approximately isentropic conditions consistent with the local surface conditions at the sight. However, in the night time outside the upwelling filament (RS \#14), the conditions strongly deviate from the idealized local isentropic conditions indicated by the vertical line. The presence of Sahara dust in the local atmosphere at this time indicates that certain air layers were advected from the continent. Note that the near-surface air is almost saturated, likely due to radiative cooling.

In Fig. 19, the entropy profiles computed from radiosonde data are compared with the sea-air entropy computed from the related measured sea-surface salinity (SSS), sea-surface temperature (SST) and sea-surface pressure, Table 1.

As another example measured over the tropical Atlantic, a radiosonde profile launched at 15:47 UTC on 7 March 2004 off South America at $9.85797^{\circ} \mathrm{N}, 33.11862^{\circ} \mathrm{E}$ is selected from the AEROSE-I cruise (Nalli et al., 2005; Morris et al., 2006) and displayed in Fig. 20. The sea-surface temperature registered by the vessel was $26.28^{\circ} \mathrm{C}$, the absolute salinity $36.22 \mathrm{~g} / \mathrm{kg}$ (Practical Salinity 36.05 ). The surface pressure was $1011.33 \mathrm{hPa}$, the relative humidity was $70.2 \%$, much lower than the related equilibrium sea-air relative humidity, Fig. 3. In the near-surface atmosphere between 1000 and $930 \mathrm{hPa}$, entropy and air fraction form an almost homogeneous layer which is characteristic for isentropically mixing or rising air. Since radiosondes cannot measure the condensed fraction of wet or ice air above the condensation level, the displayed profiles reflect the gaseous part only. In comparison to equilibrium conditions at the sea surface (indicated by the vertical lines), the ambient air had lower entropy and specific humidity.

Over most ocean regions, surface relative humidity has relatively small spatial and inter-annual variations; it is typically within $75 \%-82 \%$ during all seasons (Dai, 2006). In contrast, the global warming signal is observed in the total atmospheric moisture content over the oceans that has increased by $0.41 \mathrm{~kg} / \mathrm{m}^{2}$ per decade since 1988 (Santer et al., 2007), while at the same time the upper-ocean salinity is rising over large parts of the global ocean (Curry et al., 2003; Stott et al., 2008). This effect counteracts the increasing freshwater flux from melting glaciers and polar ice caps, as described by the "osmotic potential" of Cochrane and Cochrane (2009). Durack and Wijffels (2009) found that the surface salinity of the global ocean has generally been increasing in arid (evaporation-dominated) regions and freshening in humid (precipitation-dominated) regions, with a spatial distribution similar to that of the surface salinity anomalies relative to the spatial mean over the globe. This has led them to suggest that the global hydrological cycle has accelerated over the past few decades. The explanation for climatological trends in surface salinity in specific regions such as the brackish Baltic Sea estuary can be significantly more complicated (Feistel et al., 2008a).
Table 1. Two selected radiosonde profiles taken off Morocco in September 1992 on r/v “A. v. Humboldt”. The sea-surface salinity SSS is given on the 1978 Practical Salinity Scale (PSS-78) which is converted to the Absolute Salinity $S_{\mathrm{A}}=\mathrm{SSS} \times u_{\mathrm{PS}}$ used in this paper by the factor $u_{\mathrm{PS}}=(35.16504 / 35) \mathrm{g} \mathrm{kg}^{-1}$ (Millero et al., 2008; IOC, 2010).

\begin{tabular}{lll}
\hline Radiosonde & RS \#14 & RS \#37 \\
\hline Date & 17.09 .92 & 21.09 .92 \\
UTC & $04: 54$ & $12: 28$ \\
Lat $^{\circ} \mathrm{N}$ & 31.96 & 31.00 \\
Long $^{\circ} \mathrm{W}$ & 11.01 & 10.17 \\
Wind vel $(\mathrm{m} / \mathrm{s})$ & 9.6 & 2.7 \\
Wind dir $(\mathrm{deg})$ & 27 & 18 \\
Clouds & $\mathrm{Sc}, \mathrm{tr}, \mathrm{Cu}, \mathrm{fra}$ & $\mathrm{Ci}, \mathrm{fib}$ \\
Cover & $10 / 10$ & $1 / 10$ \\
P(hPa) & 1014.0 & 1017.8 \\
T-dry $\left({ }^{\circ} \mathrm{C}\right)$ & 21.2 & 16.9 \\
SST $\left({ }^{\circ} \mathrm{C}\right)$ & 22.3 & 17.3 \\
SSS & 36.96 & 36.23 \\
Remark & Sahara dust & dust \\
Upwelling & no & yes \\
\hline
\end{tabular}

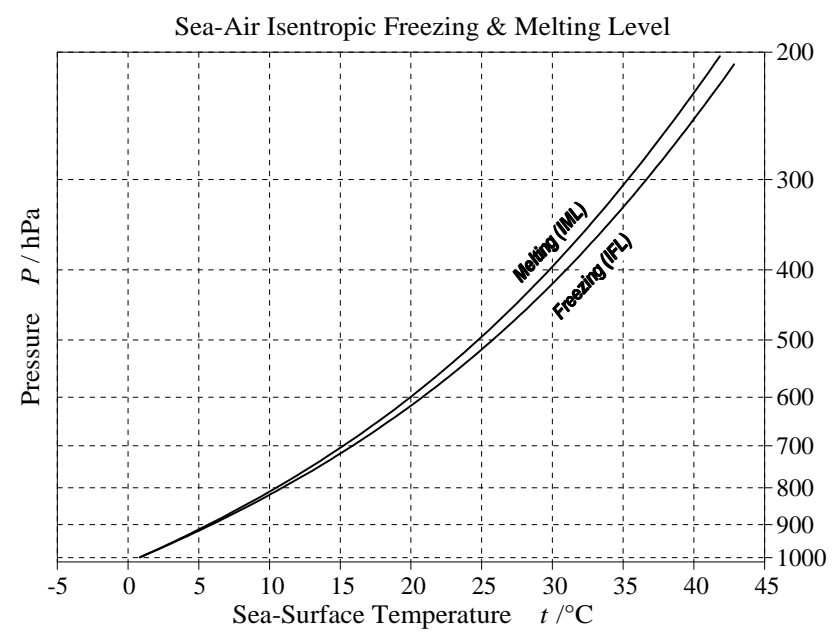

Fig. 17. Isentropic freezing level (IFL) and melting level (IML) of sea air with standard-ocean salinity $\left(35.16504 \mathrm{~g} \mathrm{~kg}^{-1}\right)$ as a function of the sea-surface temperature (SST), computed from Eqs. (9.3), (9.4) with entropy and air fraction computed from Eqs. (6.4) and (6.18). At pressures higher than IFL, the condensed part is liquid, at pressures lower than IML, the condensate is ice. Between the two curves, the solid, liquid and gas phase of water coexist. Both curves begin at the condensation level (ICL), Fig. 16, and do not reach the sea surface. Note that the local temperature (Fig. 18) is always very near $0{ }^{\circ} \mathrm{C}$ in the mixed-phase region between IML and IFL.

In non-equilibrium situations that are usually found at the air-sea interface, Figs. 19 and 20, thermodynamic fluxes are driven by Onsager forces such as the gradient of 


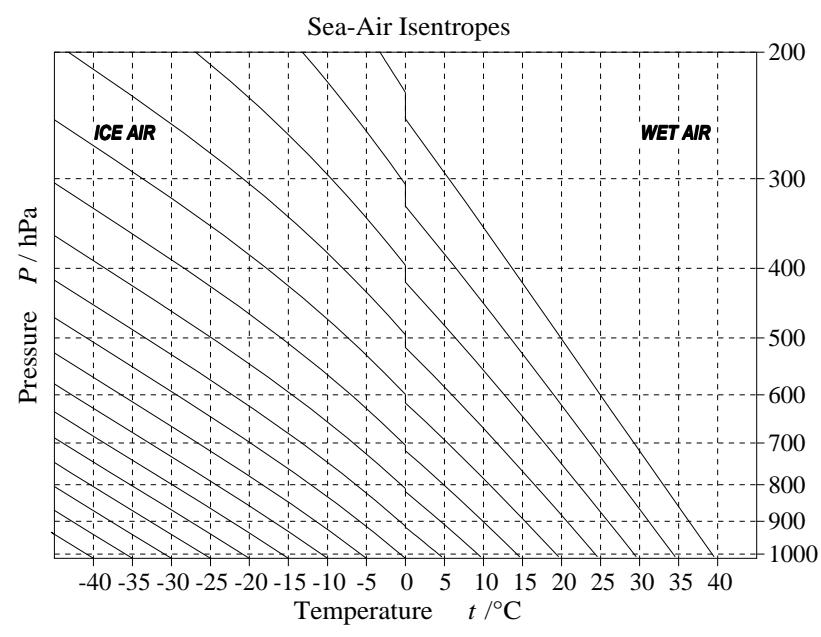

Fig. 18. Vertical isentropic sea-air profiles of temperature for different SST values between -40 and $+40^{\circ} \mathrm{C}$ at standard-ocean salinity (35.16504 $\mathrm{g} \mathrm{kg}^{-1}$ ) and sea-level pressure (101325 Pa), beyond the related isentropic condensation level shown in Fig. 16. Note that the pressure range in Fig. 16 is compressed into a very thin layer at the bottom of this plot. The isentropic transition between liquid and solid condensate, between IFL and IML in Fig. 17, has an extremely small lapse rate and appears here as a pressure jump, i.e. as an almost isothermal layer in which the solid, liquid and vapour phase of water coexist, Fig. 11b.

$\mu / T$ (Glansdorff and Prigogine, 1971; De Groot and Mazur, 1984). At the sea surface, assuming the same temperature and pressure on both sides of the interface, the dimensionless Onsager force $X_{\mathrm{SA}}\left(A, S_{\mathrm{A}}, T, P\right)$ driving the transfer of water is the difference between the chemical potentials of water in humid air and in seawater,

$X_{\mathrm{SA}}=\Delta\left(\frac{\mu}{R_{\mathrm{W}} T}\right)=\frac{\mu_{\mathrm{AV}}^{\mathrm{V}}(A, T, P)}{R_{\mathrm{W}} T}-\frac{\mu^{\mathrm{W}}\left(S_{\mathrm{A}}, T, P\right)}{R_{\mathrm{W}} T}$

It vanishes at the condensation point, $A=A^{\text {cond }}\left(S_{\mathrm{A}}, T, P\right)$, Eq. (6.4), rather than at saturation. Equation (12.1) can be expressed in terms of fugacities, Eqs. (10.10) and (10.11), in the form

$X_{\mathrm{SA}}=\ln \frac{\varphi(A)}{\varphi^{\mathrm{SA}}\left(S_{\mathrm{A}}\right)}=m_{\mathrm{SW}} M^{\mathrm{W}} \phi^{\mathrm{SW}}+\ln \varphi(A)$.

Rather than the relative humidity, Eqs. (10.2), (10.4), the sea-air Onsager force $X_{\mathrm{SA}}$, in conjunction with the formula (6.28) for the evaporation enthalpy of seawater, is relevant for the parameterization of non-equilibrium latent heat fluxes across the sea surface. In the special case of limnological applications, or below the freezing point of seawater, it reduces to $X_{\mathrm{SA}}=\ln \varphi(A)$, which corresponds to the relative humidity, $\ln \left(R H_{\mathrm{CCT}}\right)$, in the ideal-gas approximation.
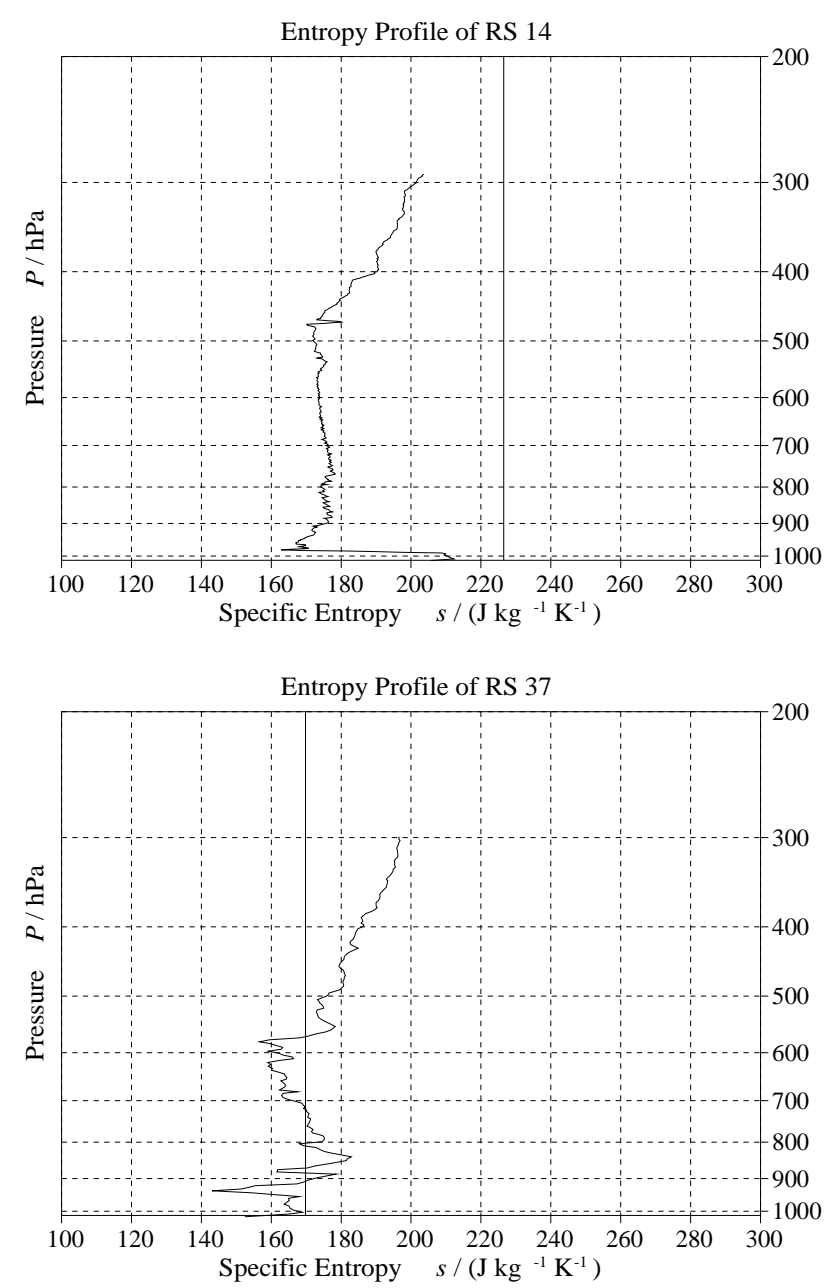

Fig. 19. Entropy of two radiosonde profiles taken on the subtropical Atlantic off Morocco, Table 1, computed from Eq. (5.16), neglecting any unknown condensed fraction, compared with the related sea-air entropy, computed from ocean surface properties by means of the equilibrium Eq. (6.4) and shown here as vertical lines. The large difference in the upper panel probably results from strong offshore wind that also carried Sahara dust observed at this time.

\section{Summary}

The broad aims of this paper are twofold. First, to provide a collection of equations for various properties of equilibria between seawater, ice and humid air, derived directly from the thermodynamic potentials for the elementary components. Second, to review information on sea air conditions and provide a best estimate of the sea-air potential function(s), expressed in different forms, based on previously published work. Very accurate and comprehensive formulations of these potentials have recently become available (IAPWS, 2008, 2009a, b; Lemmon et al., 2000; Feistel et al., 2008b) and permit the consistent computation of all their thermodynamic single-phase properties as well as of their 

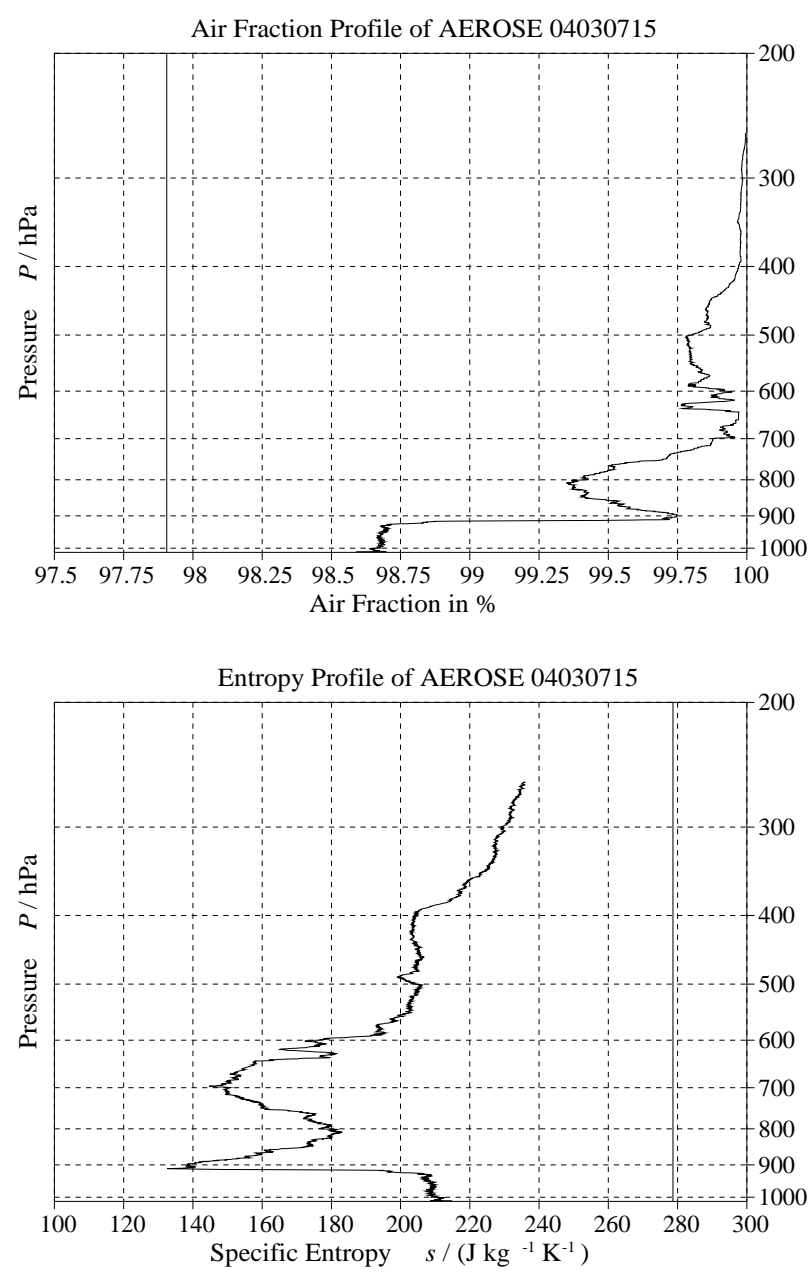

Fig. 20. Profiles of air fraction and entropy of the radiosonde profiles AEROSE 04030715 taken on 7 March 2004 15:47 UTC in the tropical Atlantic at $9.85797^{\circ} \mathrm{N}, 33.11862^{\circ} \mathrm{E}$ showing an isentropic surface layer (Courtesy Nicholas R. Nalli, NOAA). The vertical lines indicate the values computed for the humid-air part from the sea-air equilibrium at the observed sea-surface temperature of $26.28^{\circ} \mathrm{C}$ and salinity of $36.22 \mathrm{~g} / \mathrm{kg}$, Eqs. (6.4) and (6.18). The air fraction $A$ on the abscissa relates to specific humidity, $q=1-A$.

various combinations in mutual phase equilibria. The formulas focus in particular on systems having seawater as one component, such as sea ice, sea air or sea-ice air, and also cover the traditional meteorological application for the limiting case of vanishing salinity.

In this paper we suggest an approach that is conceptually (and practically) different from the common approach of using a collection of separate empirical equations for properties of interest. In particular, we describe how a small number of previously published potential functions can be consistently combined with each other and exploited mathematically to calculate the wide range of thermodynamic properties of interest in oceanography and meteorology. Most of the equations that we consider are expressions of exact thermody- namic relations. Consequently, it is a fundamental feature of our approach that the quantitative accuracy of the thermodynamic potentials used as "inputs" is not relevant to the mathematical correctness of the fundamental thermodynamic relations which are the primary subject of this paper. Because of our focus on general relations that follow from exact thermodynamic principles, we do not discuss the correctness of any particular selection taken from the various published empirical geophysical correlation equations. In most cases, the verifications of those empirical equations have been achieved through many practical applications as discussed in the literature. Similarly, we do not discuss the mutual consistency of examples for pairwise and higher-order combinations of those correlations in order to, say, demonstrate the higher accuracy of the potential functions we employ.

The relations considered here constitute the mathematical "processing pipeline" applied to extract from the given "input" the various special properties of interest. If improved formulations for any of the chosen potential functions become available in the future, these "inputs" may be substituted for our current choices without any fundamental changes of the relations collected and published in this paper. Nevertheless, we note that the potential functions that we recommend here are (or are planned to be) formulated in officially endorsed IAPWS documents and represent internationally recognized standards that possess distinctively high reliability and accuracy.

Starting from those very few but comprehensive formulations, all thermodynamic properties can be derived (within the range of validity and the related uncertainty) in a consistent and highly accurate way. Based on this approach, studies of different authors using these properties will be much more directly comparable. We also note that although limitations of space and time have not permitted it, a virtually unlimited set of tailored correlation equations can be derived for special applications from the exact thermodynamic relations and the chosen potential functions, even for properties for which direct measurements are unavailable or show significant uncertainties (e.g., sublimation pressures at very low temperatures or freezing points at high pressures).

It should be noted that the two parts, i.e., the empirical primary standard and the set of rigorous thermodynamic formulas, can be advanced essentially independently. That is, new functions based on the fundamental thermodynamic relations can be introduced into user libraries (e.g., Feistel et al., 2009; Wright et al., 2009) and/or new or extended versions of the empirically determined thermodynamic potentials can be introduced without directly affecting the other part. All that is required is that a standard interface be established to communicate information between the two parts. For example, a mass-based Gibbs function with its first and second derivatives, inputs of Absolute Salinity, absolute ITS-90 temperature and absolute pressure can be replaced by a revised or completely new function with these same properties without requiring any other changes. 
The Helmholtz function (5.2) of humid air is composed here of the Helmholtz functions of dry air and of fluid water with additional second and third cross virial coefficients describing the air-water interaction, i.e. non-ideal properties of humid air, Fig. 1.

The Gibbs function (6.2) of sea-air describes a composite system of seawater and humid air in mutual thermodynamic equilibrium, controlled by the Clausius-Clapeyron Eq. (6.7). Subsequently, formulas for several properties of sea air are derived from the Gibbs function, in particular the latent heat of seawater, Eq. (6.28), Fig. 5.

In the limit of vanishing salinity, sea air reduces to wet air, i.e. saturated humid air in equilibrium with condensed water. The Gibbs function of wet air, Eqs. (7.1) and (7.4), provides various properties of central interest in meteorology, in particular the saturation point (7.5), Figs. 8 and 9. The humid-air part of sea air is subsaturated, Fig. 3. The subsaturation of sea air can be quantified by the relative humidity, Eqs. (10.2) and (10.4), in two different standard definitions, or by the relative fugacity, Eqs. (10.10) and (10.14).

Below the freezing temperature of seawater, sea air contains ice Ih as a third phase. Its Gibbs function, Eq. (8.2), and the two Clausius-Clapeyron equations, Eqs. (8.8) and (6.7), are used to derive equations for related properties. The humid-air part of sea-ice air is always saturated because the equilibrium between ice and humid air is independent of additionally present seawater. A special situation occurs between the freezing points of water and of sea-water where neither ice nor liquid water can form a stable phase. In this temperature interval, the humid-air part of sea air is subsaturated, Fig. 10. The latent heat, Eq. (8.18), of sea-ice air, i.e. of water evaporating at constant pressure from sea ice, is a complex three-phase expression which, beyond the sublimation enthalpy, includes the melting heat resulting from the temperature change of ice and the salinity change of the brine.

At zero salinity, sea-ice air reduces to ice air with only two phases, ice and humid air, except for a small temperaturepressure window in which ice, liquid water and water vapour coexist in the presence of air. This "triple-point" atmosphere is referred to as wet ice air, Fig. 11, and forms an almost isothermal layer of isentropically lifted air between the melting and the freezing level, Figs. 17, 18. In the two-phase region of ice air, saturation is defined with respect to ice, Fig. 13.

The properties provided in this paper can be computed from the currently available potential functions for humid air, fluid water, ice and seawater and the related equations will not require modification as the potentials are improved. Based on functions available from the literature as repeated in detail in the appendix, a new seawater library (Feistel et al., 2009; Wright et al., 2009) has currently been developed as source code in Fortran and Visual Basic, with later versions planned also in MatLab and $\mathrm{C} / \mathrm{C}++$, and will offer easy
Table A1. Chemical composition of sea salt (Millero et al., 2008). Mole fractions are exact by definition, mass fractions are rounded to seven digits behind the period. Uncertainties of the molar masses (Wieser, 2006) are given in brackets.

\begin{tabular}{llll}
\hline Solute & $\begin{array}{l}\text { Mole } \\
\text { fraction }\end{array}$ & $\begin{array}{l}\text { Mass } \\
\text { fraction }\end{array}$ & $\begin{array}{l}\text { Molar mass } \\
\mathrm{g} \mathrm{mol}^{-1}\end{array}$ \\
\hline $\mathrm{Na}^{+}$ & 0.4188071 & 0.3065958 & $22.98976928(2)$ \\
$\mathrm{Mg}^{2+}$ & 0.0471678 & 0.0365055 & $24.3050(6)$ \\
$\mathrm{Ca}^{2+}$ & 0.0091823 & 0.0117186 & $40.078(4)$ \\
$\mathrm{K}^{+}$ & 0.0091159 & 0.0113495 & $39.0983(1)$ \\
$\mathrm{Sr}^{2+}$ & 0.0000810 & 0.0002260 & $87.62(1)$ \\
$\mathrm{Cl}^{-}$ & 0.4874839 & 0.5503396 & $35.453(2)$ \\
$\mathrm{SO}_{4}^{2-}$ & 0.0252152 & 0.0771319 & $96.0626(50)$ \\
$\mathrm{HCO}_{3}^{-}$ & 0.0015340 & 0.0029805 & $61.01684(96)$ \\
$\mathrm{Br}^{-}$ & 0.0007520 & 0.0019134 & $79.904(1)$ \\
$\mathrm{CO}_{3}^{2-}$ & 0.0002134 & 0.0004078 & $60.0089(10)$ \\
$\left.\mathrm{B}^{2} \mathrm{OH}\right)_{4}^{-}$ & 0.0000900 & 0.0002259 & $78.8404(70)$ \\
$\mathrm{F}^{-}$ & 0.0000610 & 0.0000369 & $18.9984032(5)$ \\
$\mathrm{OH}^{-}$ & 0.0000071 & 0.0000038 & $17.00733(7)$ \\
$\left.\mathrm{B}^{2} \mathrm{OH}\right)_{3}$ & 0.0002807 & 0.0005527 & $61.8330(70)$ \\
$\mathrm{CO}_{2}$ & 0.0000086 & 0.0000121 & $44.0095(9)$ \\
$\mathrm{Sea} \mathrm{salt}^{-}$ & 1.0000000 & 1.0000000 & $31.404(2)$ \\
\hline
\end{tabular}

access to sea-air as well as fluid water, ice and seawater properties.

\section{Appendix A}

\section{Composition of sea salt and dry air}

The chemical compositions of sea salt and dry air are given in Tables A1 and A2, respectively.

\section{Appendix B}

\section{Thermodynamic potential of dry air}

The specific Helmholtz energy for dry air is (Lemmon et al., 2000),

$f^{\mathrm{A}}\left(T, \rho^{\mathrm{A}}\right)=R_{\mathrm{A}}^{\mathrm{L}} T\left[\alpha^{\mathrm{id}}(\tau, \delta)+\alpha^{\mathrm{res}}(\tau, \delta)\right]$,

where $R_{\mathrm{A}}^{\mathrm{L}}=R^{\mathrm{L}} / M^{\mathrm{A}, \mathrm{L}}$ is the specific gas constant of air, $M^{\mathrm{A}, \mathrm{L}}=28.9586 \mathrm{~g} \mathrm{~mol}^{-1}$ and $R^{\mathrm{L}}=8.31451 \mathrm{~J} \mathrm{~mol}^{-1} \mathrm{~K}^{-1}$ are the molar mass and gas constant used by Lemmon et al. (2000). Note that these values differ slightly from the most recent ones given in Table A1. The function $\alpha^{\text {id }}(\tau, \delta)$ is the ideal-gas part, 
Table A2. Chemical composition of dry air. Mole fractions from Picard et al. (2008) except for $\mathrm{N}_{2}$ which was adjusted by subtracting all other mole fractions from 1 (Picard et al., 2008). Uncertainties of the molar masses (Wieser, 2006) are given in brackets.

\begin{tabular}{llll}
\hline & $\begin{array}{l}\text { Mole } \\
\text { fraction }\end{array}$ & $\begin{array}{l}\text { Mass } \\
\text { fraction }\end{array}$ & $\begin{array}{l}\text { Molar mass } \\
\mathrm{g} \mathrm{mol}^{-1}\end{array}$ \\
\hline $\mathrm{N}_{2}$ & 0.7808479 & 0.75518473 & $28.0134(3)$ \\
$\mathrm{O}_{2}$ & 0.2093900 & 0.23131860 & $31.9988(4)$ \\
$\mathrm{Ar}$ & 0.0093320 & 0.01287036 & $39.948(1)$ \\
$\mathrm{CO}_{2}$ & 0.0004000 & 0.00060775 & $44.0095(9)$ \\
$\mathrm{Ne}$ & 0.0000182 & 0.00001268 & $20.1797(6)$ \\
$\mathrm{He}$ & 0.0000052 & 0.00000072 & $4.002602(2)$ \\
$\mathrm{CH}$ & 0.0000015 & 0.00000083 & $16.04246(81)$ \\
$\mathrm{Kr}$ & 0.0000011 & 0.00000318 & $83.798(2)$ \\
$\mathrm{H}_{2}$ & 0.0000005 & 0.00000003 & $2.01588(10)$ \\
$\mathrm{N}_{2} \mathrm{O}$ & 0.0000003 & 0.00000046 & $44.0128(4)$ \\
$\mathrm{CO}$ & 0.0000002 & 0.00000019 & $28.0101(9)$ \\
$\mathrm{Xe}$ & 0.0000001 & 0.00000045 & $131.293(6)$ \\
$\mathrm{Air}$ & 1.0000000 & 0.99999998 & $28.96546(33)$ \\
\hline
\end{tabular}

$$
\begin{aligned}
& \alpha^{\mathrm{id}}(\tau, \delta)=\ln \delta+\sum_{i=1}^{5} n_{i}^{0} \tau^{i-4}+n_{6}^{0} \tau^{1.5}+n_{7}^{0} \ln \tau \\
& \quad+n_{8}^{0} \ln \left[1-\exp \left(-n_{11}^{0} \tau\right)\right]+n_{9}^{0} \ln \left[1-\exp \left(-n_{12}^{0} \tau\right)\right] \\
& \quad+n_{10}^{0} \ln \left[2 / 3+\exp \left(n_{13}^{0} \tau\right)\right]
\end{aligned}
$$

and $\alpha^{\text {res }}(\tau, \delta)$ is the residual part,

$\alpha^{\mathrm{res}}(\tau, \delta)=\sum_{k=1}^{10} n_{k} \delta^{i_{k}} \tau^{j_{k}}+\sum_{k=11}^{19} n_{k} \delta^{i_{k}} \tau^{j_{k}} \exp \left(-\delta^{l_{k}}\right)$.

Here, the reduced variables are $\tau=132.6312 \mathrm{~K} / T, \delta=$ $\rho^{\mathrm{A}} /\left(10.4477 \mathrm{moldm}^{-3} \times M^{\mathrm{A}, \mathrm{L}}\right)$, and the coefficients are given in Tables B1 and B2. Numerical check values are available from Table I2, Appendix I.

The temperature scale is ITS-90. Equation (B1) describes reliable experimental data available for $60-873 \mathrm{~K}$ and to $70 \mathrm{MPa}$, and predicts air properties even up to $2000 \mathrm{~K}$ and $2000 \mathrm{MPa}$. Here, we are interested only in the ranges in which air is in its gas phase, either at $T>T_{\mathrm{c}}$, or $T$ in the interval $T_{\mathrm{c}}>T>T_{\mathrm{Liq}}(P)$, between the critical temperature $T_{\mathrm{c}}$ and the dewpoint temperature $T_{\mathrm{Liq}}$ of liquid air. The critical point of air is at $T_{\mathrm{c}}=132.5306 \mathrm{~K}, P_{\mathrm{c}}=3.7860 \mathrm{MPa}$, $\rho_{\mathrm{c}}=11.8308 \mathrm{~mol} \mathrm{dm}^{-3} \times M^{\mathrm{A}, \mathrm{L}} \approx 342.685 \mathrm{~kg} \mathrm{~m}^{-3}$. The dewpoint curve $T_{\mathrm{Liq}}(P)$ is given by Lemmon et al. (2000).
Table B1. Coefficients and exponents for the ideal-gas part (B2) for dry air (Lemmon et al., 2000). Here, we have adjusted the coefficients $n_{4}^{0}$ and $n_{5}^{0}$ to the reference state conditions of zero entropy and zero enthalpy of dry air at the standard ocean surface state, $0^{\circ} \mathrm{C}$ and $101325 \mathrm{~Pa}$. The originally published values are $n_{4}^{0}=-13.841928076$ and $n_{5}^{0}=17.275266575$.

\begin{tabular}{llll}
\hline$i$ & $n_{i}^{0}$ & $i$ & $n_{i}^{0}$ \\
\hline 1 & $+0.60571940000 \times 10^{-7}$ & 8 & +0.79130950900 \\
2 & $-0.21027476900 \times 10^{-4}$ & 9 & +0.21223676800 \\
3 & $-0.15886071600 \times 10^{-3}$ & 10 & -0.19793890400 \\
4 & $+0.97450251743948 \times 10$ & 11 & $+0.25363650000 \times 10^{2}$ \\
5 & $+0.100986147428912 \times 10^{2}$ & 12 & $+0.16907410000 \times 10^{2}$ \\
6 & $-0.19536342000 \times 10^{-3}$ & 13 & $+0.87312790000 \times 10^{2}$ \\
7 & $+0.24908880320 \times 10$ & & \\
\hline
\end{tabular}

\section{Appendix C}

\section{Thermodynamic potential of fluid water}

The specific Helmholtz energy for fluid (gaseous and liquid) water is (IAPWS, 2009a; Wagner and Pruß, 2002),

$f^{\mathrm{V}}\left(T, \rho^{\mathrm{V}}\right)=f^{\mathrm{V}, \mathrm{id}}\left(T, \rho^{\mathrm{V}}\right)+R_{\mathrm{W}}^{95} T \phi^{\mathrm{res}}(\tau, \delta)$,

where $f^{\mathrm{V}, \mathrm{id}}\left(T, \rho^{\mathrm{V}}\right)$ is the ideal-gas part, Eq. (C2), $R_{\mathrm{W}}^{95}=461.51805 \mathrm{~J} \mathrm{~kg}^{-1} \mathrm{~K}^{-1}$ is the specific gas constant of $\mathrm{H}_{2} \mathrm{O}$ used in IAPWS-95, and $\phi^{\text {res }}(\tau, \delta)$ is the dimensionless residual part consisting of 56 terms, available from Eq. (C5) and the Tables C2-C4. Note that the gas constant used here differs from the most recent value, $R_{\mathrm{W}}=R / M^{\mathrm{W}}=461.52364 \mathrm{~J} \mathrm{~kg}^{-1} \mathrm{~K}^{-1}$, where $M^{\mathrm{W}}=18.015268 \mathrm{~g} \mathrm{~mol}^{-1}$ is the molar mass of water (IAPWS, 2005).

The ideal-gas part, $f^{\mathrm{V}, \text { id }}\left(T, \rho^{\mathrm{V}}\right)$, of the specific Helmholtz energy for water vapour is (modified from IAPWS, 2009a; Wagner and Pruß, 2002)

$f^{\mathrm{V}, \mathrm{id}}\left(T, \rho^{\mathrm{V}}\right)=R_{\mathrm{W}}^{95} T\left[\phi^{0}(\tau, \delta)+\phi^{\mathrm{ex}}(\tau)\right]$.

The function $\phi^{0}(\tau, \delta)$ was obtained from an equation for the specific isobaric heat capacity developed by J.R. Cooper (1982) and reads

$\phi^{0}(\tau, \delta)=\ln \delta+n_{1}^{0}+n_{2}^{0} \tau+n_{3}^{0} \ln \tau+\sum_{i=4}^{8} n_{i}^{0} \ln \left(1-e^{-\gamma_{i}^{0} \tau}\right)$.

Reduced density $\delta=\rho^{\mathrm{V}} / \rho_{\mathrm{c}}$ and temperature $\tau=T_{\mathrm{c}} / T$ are specified by $\rho_{\mathrm{c}}=322 \mathrm{kgm}^{-3}, T_{\mathrm{c}}=647.096 \mathrm{~K}$. The coefficients of Eq. (C3) are available from Table C1. The IAPWS95 reference state conditions define the internal energy and the entropy of liquid water to be zero at the triple point. A highly accurate numerical implementation of these conditions gave the values rounded to 16 digits for the adjustable coefficients $n_{1}^{\circ}=-8.320446483749693$ and $n_{2}^{\circ}=$ 6.683210527593226 (Feistel et al., 2008b). 
Table B2. Coefficients and exponents for the fundamental Eq. (B3) for dry air (Lemmon et al., 2000).

\begin{tabular}{llllllllll}
\hline$k$ & $i_{k}$ & $j_{k}$ & $l_{k}$ & $n_{k}$ & $k$ & $i_{k}$ & $j_{k}$ & $l_{k}$ & $n_{k}$ \\
\hline 1 & 1 & 0 & 0 & +0.118160747229 & 11 & 1 & 1.6 & 1 & -0.101365037912 \\
2 & 1 & 0.33 & 0 & +0.713116392079 & 12 & 3 & 0.8 & 1 & -0.173813690970 \\
3 & 1 & 1.01 & 0 & $-0.161824192067 \times 10$ & 13 & 5 & 0.95 & 1 & $-0.472103183731 \times 10^{-1}$ \\
4 & 2 & 0 & 0 & $+0.714140178971 \times 10^{-1}$ & 14 & 6 & 1.25 & 1 & $-0.122523554253 \times 10^{-1}$ \\
5 & 3 & 0 & 0 & $-0.865421396646 \times 10^{-1}$ & 15 & 1 & 3.6 & 2 & -0.146629609713 \\
6 & 3 & 0.15 & 0 & +0.134211176704 & 16 & 3 & 6 & 2 & $-0.316055879821 \times 10^{-1}$ \\
7 & 4 & 0 & 0 & $+0.112626704218 \times 10^{-1}$ & 17 & 11 & 3.25 & 2 & $+0.233594806142 \times 10^{-3}$ \\
8 & 4 & 0.2 & 0 & $-0.420533228842 \times 10^{-1}$ & 18 & 1 & 3.5 & 3 & $+0.148287891978 \times 10^{-1}$ \\
9 & 4 & 0.35 & 0 & $+0.349008431982 \times 10^{-1}$ & 19 & 3 & 15 & 3 & $-0.938782884667 \times 10^{-2}$ \\
10 & 6 & 1.35 & 0 & $+0.164957183186 \times 10^{-3}$ & & & & & \\
\hline
\end{tabular}

Table C1. Coefficients of the ideal part (C3). Note that the originally published values (Wagner and Pruß, 2002) of the adjustable coefficients $n_{1}^{\circ}$ and $n_{2}^{\circ}$ are slightly different from those given here. Those given here are preferred (IAPWS, 2009b; Feistel et al., 2008b).

\begin{tabular}{lll}
\hline$i$ & $n_{i}^{0}$ & $\gamma_{i}^{0}$ \\
\hline 1 & -8.32044648374969 & \\
2 & +6.68321052759323 & \\
3 & +3.00632 & \\
4 & +0.012436 & 1.28728967 \\
5 & +0.97315 & 3.53734222 \\
6 & +1.2795 & 7.74073708 \\
7 & +0.96956 & 9.24437796 \\
8 & +0.24873 & 27.5075105 \\
\hline
\end{tabular}

The temperature $T$ is measured on the ITS-90 scale. The range of validity is $130-1273 \mathrm{~K}$ without the extension $(\mathrm{C} 4)$, i.e. with $\phi^{\mathrm{ex}}(\tau) \equiv 0$. The range can be extended to include the region $50-130 \mathrm{~K}$ with a correction function added in this temperature range,

$$
\begin{aligned}
& \phi^{\mathrm{ex}}(\tau) \\
& =E \times\left(-\frac{1}{2 \tau}-\frac{3}{\varepsilon^{2}}(\tau+\varepsilon) \ln \frac{\tau}{\varepsilon}-\frac{9}{2 \varepsilon}+\frac{9 \tau}{2 \varepsilon^{2}}+\frac{\tau^{2}}{2 \varepsilon^{3}}\right),
\end{aligned}
$$

at $50 \mathrm{~K} \leq T \leq 130 \mathrm{~K}$,

where $T_{\mathrm{E}}=130 \mathrm{~K}, E=0.278296458178592, \varepsilon=T_{\mathrm{c}} / T_{\mathrm{e}}$. At $\tau=\varepsilon, \phi^{\mathrm{ex}}(\tau)$ is zero, as well as its first, second, third and fourth temperature derivatives. This correction has been determined such that when applied to Cooper's formula used in IAPWS-95, it results in a fit to the heat capacity data of Woolley (1980) between 50 and $130 \mathrm{~K}$ with an r.m.s. deviation of $6 \times 10^{-4}$ in $c_{p} / R_{\mathrm{W}}$. This extension formula has been developed particularly for implementation in the source-code library and is not published elsewhere.
The residual part of Eq. (C1) has the form

$$
\begin{aligned}
& \phi^{\mathrm{res}}=\sum_{i=1}^{7} n_{i} \delta^{d_{i}} \tau^{t_{i}}+\sum_{i=8}^{51} n_{i} \delta^{d_{i}} \tau^{t_{i}} \exp \left(-\delta^{c_{i}}\right) \\
& +\sum_{i=52}^{54} n_{i} \delta^{d_{i}} \tau^{t_{i}} \exp \left(-\alpha_{i}\left(\delta-\varepsilon_{i}\right)^{2}-\beta_{i}\left(\tau-\gamma_{i}\right)^{2}\right) \\
& \quad+\sum_{i=55}^{56} n_{i} \Delta^{b_{i}} \delta \psi
\end{aligned}
$$

with the abbreviations

$\Delta=\theta^{2}+B_{i}|\delta-1|^{2 a_{i}}$,

$\theta=1-\tau+A_{i}|\delta-1|^{\frac{1}{\beta_{i}}}$,

and

$\psi=\exp \left(-C_{i}(\delta-1)^{2}-D_{i}(\tau-1)^{2}\right)$.

Equation (C1) is valid between 50 and $1273 \mathrm{~K}$ and for pressures up to $1000 \mathrm{MPa}$ in the stable single-phase region of fluid water. Uncertainty estimates are available from IAPWS (2009a) and Wagner and Pruß (2002). Numerical check values are available from Table I2, Appendix I.

\section{Appendix D}

\section{Thermodynamic potential of humid air}

The Helmholtz energy $F^{\mathrm{AV} \text {,id }}$ of humid air in the ideal-gas approximation (H3) is additive with respect to its compo-

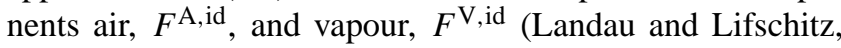
1987, §93),

$F^{\mathrm{AV}, \text { id }}\left(N_{\mathrm{A}}, N_{\mathrm{V}}, T, V\right)=F^{\mathrm{A}, \mathrm{id}}\left(N_{\mathrm{A}}, T, V\right)+F^{\mathrm{V}, \mathrm{id}}\left(N_{\mathrm{V}}, T, V\right) .(\mathrm{D} 1)$

Here, $N_{\mathrm{A}}$ and $N_{\mathrm{V}}$ are the numbers of air and vapour particles in the volume $V$. The additivity of the Helmholtz energy (D1) is consistent with the entropy of mixing of ideal 
Table C2. Coefficients of the residual part (C5).

\begin{tabular}{|c|c|c|c|c|}
\hline$i$ & $c_{i}$ & $d_{i}$ & $t_{i}$ & $n_{i}$ \\
\hline 1 & 0 & 1 & -0.5 & +0.012533547935523 \\
\hline 2 & 0 & 1 & 0.875 & +7.8957634722828 \\
\hline 3 & 0 & 1 & 1 & -8.7803203303561 \\
\hline 4 & 0 & 2 & 0.5 & +0.31802509345418 \\
\hline 5 & 0 & 2 & 0.75 & -0.26145533859358 \\
\hline 6 & 0 & 3 & 0.375 & $-7.8199751687981 \times 10^{-3}$ \\
\hline 7 & 0 & 4 & 1 & $+8.8089493102134 \times 10^{-3}$ \\
\hline 8 & 1 & 1 & 4 & -0.66856572307965 \\
\hline 9 & 1 & 1 & 6 & +0.20433810950965 \\
\hline 10 & 1 & 1 & 12 & $-6.6212605039687 \times 10^{-5}$ \\
\hline 11 & 1 & 2 & 1 & -0.19232721156002 \\
\hline 12 & 1 & 2 & 5 & -0.25709043003438 \\
\hline 13 & 1 & 3 & 4 & +0.16074868486251 \\
\hline 14 & 1 & 4 & 2 & -0.040092828925807 \\
\hline 15 & 1 & 4 & 13 & $+3.9343422603254 \times 10^{-7}$ \\
\hline 16 & 1 & 5 & 9 & $-7.5941377088144 \times 10^{-6}$ \\
\hline 17 & 1 & 7 & 3 & $+5.6250979351888 \times 10^{-4}$ \\
\hline 18 & 1 & 9 & 4 & $-1.5608652257135 \times 10^{-5}$ \\
\hline 19 & 1 & 10 & 11 & $+1.1537996422951 \times 10^{-9}$ \\
\hline 20 & 1 & 11 & 4 & $+3.6582165144204 \times 10^{-7}$ \\
\hline 21 & 1 & 13 & 13 & $-1.3251180074668 \times 10^{-12}$ \\
\hline 22 & 1 & 15 & 1 & $-6.2639586912454 \times 10^{-10}$ \\
\hline 23 & 2 & 1 & 7 & -0.10793600908932 \\
\hline 24 & 2 & 2 & 1 & +0.017611491008752 \\
\hline 25 & 2 & 2 & 9 & +0.22132295167546 \\
\hline 26 & 2 & 2 & 10 & -0.40247669763528 \\
\hline 27 & 2 & 3 & 10 & +0.58083399985759 \\
\hline 28 & 2 & 4 & 3 & $+4.9969146990806 \times 10^{-3}$ \\
\hline 29 & 2 & 4 & 7 & -0.031358700712549 \\
\hline 30 & 2 & 4 & 10 & -0.74315929710341 \\
\hline 31 & 2 & 5 & 10 & +0.4780732991548 \\
\hline 32 & 2 & 6 & 6 & +0.020527940895948 \\
\hline 33 & 2 & 6 & 10 & -0.13636435110343 \\
\hline 34 & 2 & 7 & 10 & +0.014180634400617 \\
\hline 35 & 2 & 9 & 1 & $+8.3326504880713 \times 10^{-3}$ \\
\hline 36 & 2 & 9 & 2 & -0.029052336009585 \\
\hline 37 & 2 & 9 & 3 & +0.038615085574206 \\
\hline 38 & 2 & 9 & 4 & -0.020393486513704 \\
\hline 39 & 2 & 9 & 8 & $-1.6554050063734 \times 10^{-3}$ \\
\hline 40 & 2 & 10 & 6 & $+1.9955571979541 \times 10^{-3}$ \\
\hline 41 & 2 & 10 & 9 & $+1.5870308324157 \times 10^{-4}$ \\
\hline 42 & 2 & 12 & 8 & $-1.638856834253 \times 10^{-5}$ \\
\hline 43 & 3 & 3 & 16 & +0.043613615723811 \\
\hline 44 & 3 & 4 & 22 & +0.034994005463765 \\
\hline 45 & 3 & 4 & 23 & -0.076788197844621 \\
\hline 46 & 3 & 5 & 23 & +0.022446277332006 \\
\hline 47 & 4 & 14 & 10 & $-6.2689710414685 \times 10^{-5}$ \\
\hline 48 & 6 & 3 & 50 & $-5.5711118565645 \times 10^{-10}$ \\
\hline 49 & 6 & 6 & 44 & -0.19905718354408 \\
\hline 50 & 6 & 6 & 46 & +0.31777497330738 \\
\hline 51 & 6 & 6 & 50 & -0.11841182425981 \\
\hline
\end{tabular}

Ocean Sci., 6, 91-141, 2010
Table C3. Coefficients of the residual part (C5).

\begin{tabular}{rrrrrrrr}
\hline$i$ & $d_{i}$ & $t_{i}$ & $n_{i}$ & $\alpha_{i}$ & $\beta_{i}$ & $\gamma_{i}$ & $\varepsilon_{i}$ \\
\hline 52 & 3 & 0 & -31.306260323435 & 20 & 150 & 1.21 & 1 \\
53 & 3 & 1 & 31.546140237781 & 20 & 150 & 1.21 & 1 \\
54 & 3 & 4 & -2521.3154341695 & 20 & 250 & 1.25 & 1 \\
\hline
\end{tabular}

Table C4. Coefficients of the residual part (C5).

\begin{tabular}{rrrrrrrrr}
\hline$i$ & $a_{i}$ & $b_{i}$ & $B_{i}$ & $n_{i}$ & $C_{i}$ & $D_{i}$ & $A_{i}$ & $\beta_{i}$ \\
\hline 55 & 3.5 & 0.85 & 0.2 & -0.14874640856724 & 28 & 700 & 0.32 & 0.3 \\
56 & 3.5 & 0.95 & 0.2 & 0.31806110878444 & 32 & 800 & 0.32 & 0.3 \\
\hline
\end{tabular}

gases, Eq. (H9). At higher densities, the corresponding Helmholtz energy of the real-gas mixture is

$$
\begin{aligned}
& F^{\mathrm{AV}}\left(N_{\mathrm{A}}, N_{\mathrm{V}}, T, V\right)=F^{\mathrm{A}}\left(N_{\mathrm{A}}, T, V\right)+F^{\mathrm{V}}\left(N_{\mathrm{V}}, T, V\right) \\
& \quad-k T \ln \frac{Q^{\mathrm{AV}}}{Q^{\mathrm{A}} Q^{\mathrm{V}}} .
\end{aligned}
$$

The configuration integrals $Q^{\mathrm{A}}, Q^{\mathrm{V}}$ and $Q^{\mathrm{AV}}$ of the canonical partition function are defined in terms of the pairinteraction potentials $u$ between the particles of air at the positions $\mathbf{r}^{\mathrm{A}}$ and those of vapour at $\mathbf{r}^{\mathrm{V}}$, as $(\beta=1 / k T)$ :

$$
\begin{aligned}
& Q^{\mathrm{AV}}=\int_{V}\left(\frac{\mathrm{d} \mathbf{r}^{\mathrm{A}}}{V}\right)^{N_{\mathrm{A}}}\left(\frac{\mathrm{d} \mathbf{r}^{\mathrm{V}}}{V}\right)^{N_{\mathrm{V}}} \\
& \exp \left\{-\beta \sum_{i<j}^{N_{\mathrm{A}}} u_{\mathrm{AA}}\left(\mathbf{r}_{i}^{\mathrm{A}}, \mathbf{r}_{j}^{\mathrm{A}}\right)-\beta \sum_{i<j}^{N_{\mathrm{V}}} u \mathrm{VV}\left(\mathbf{r}_{i}^{\mathrm{V}}, \mathbf{r}_{j}^{\mathrm{V}}\right)\right. \\
&\left.-\beta \sum_{i=1}^{N_{\mathrm{A}}} \sum_{j=1}^{N_{\mathrm{V}}} u_{\mathrm{AV}}\left(\mathbf{r}_{i}^{\mathrm{A}}, \mathbf{r}_{j}^{\mathrm{V}}\right)\right\} \\
& Q^{\mathrm{A}}=\int_{V}\left(\frac{\mathrm{d} \mathbf{r}^{\mathrm{A}}}{V}\right)^{N_{\mathrm{A}}} \exp \left\{-\beta \sum_{i<j}^{N_{\mathrm{A}}} u_{\mathrm{AA}}\left(\mathbf{r}_{i}^{\mathrm{A}}, \mathbf{r}_{j}^{\mathrm{A}}\right)\right\} \\
& Q^{\mathrm{V}}=\int_{V}\left(\frac{\mathrm{d} \mathbf{r}^{\mathrm{V}}}{V}\right)^{N_{\mathrm{V}}} \exp \left\{-\beta \sum_{i<j}^{N_{\mathrm{V}}} u_{\mathrm{VV}}\left(\mathbf{r}_{i}^{\mathrm{V}}, \mathbf{r}_{j}^{\mathrm{V}}\right)\right\} .
\end{aligned}
$$

Here, $\left(\mathrm{d} \mathbf{r}^{\mathrm{A}}\right)^{N_{\mathrm{A}}}$ is the shorthand notation for the $N_{\mathrm{A}}$ nested volume integrations $\mathrm{d} \mathbf{r}_{1}^{\mathrm{A}} \mathrm{d} \mathbf{r}_{2}^{\mathrm{A}} \ldots \mathrm{d} \mathbf{r}_{N_{\mathrm{A}}}^{\mathrm{A}}$, and similarly for $\left(\mathrm{d} \mathbf{r}^{\mathrm{V}}\right)^{N_{\mathrm{V}}}$. For the pure components, $F^{\mathrm{A}}$ and $F^{\mathrm{V}}$, the formulations of Lemmon et al. (2000) and IAPWS-95 are available from the literature. They provide empirical approximate expressions for the integrals (D4), $Q^{\mathrm{A}}$, and (D5), $Q^{\mathrm{V}}$, valid up to very high densities of either dry air or pure water. For the mixed term, only the lowest-order cross-virial coefficients 
Table D1. Coefficients of the virial coefficients, Eqs. (D16-D18).

\begin{tabular}{lllll}
\hline$i$ & $a_{i}$ & $b_{i}$ & $c_{i}$ & $d_{i}$ \\
\hline 0 & $+0.482737 \times 10^{-3}$ & $-0.10728876 \times 10^{2}$ & & \\
1 & +0.105678 & $+0.347802 \times 10^{4}$ & +66.5687 & -0.237 \\
2 & $-0.656394 \times 10^{2}$ & $-0.383383 \times 10^{6}$ & -238.834 & -1.048 \\
3 & $+0.294442 \times 10^{5}$ & $+0.33406 \times 10^{8}$ & -176.755 & -3.183 \\
4 & $-0.319317 \times 10^{7}$ & & & \\
\hline
\end{tabular}

are known. We introduce the binary air-vapour correlation function,

$h_{i j}^{\mathrm{AV}}=\exp \left(-\beta u_{\mathrm{AV}}\left(\mathbf{r}_{i}^{\mathrm{A}}, \mathbf{r}_{j}^{\mathrm{V}}\right)\right)-1$,

and write the mixed exponential of Eq. (D3) in the form of the cluster expansion (Kammer and Schwabe, 1971; Goodstein, 1975)

$$
\begin{aligned}
& \exp \left\{-\beta \sum_{i=1}^{N_{\mathrm{A}}} \sum_{j=1}^{N_{\mathrm{V}}} u_{\mathrm{AV}}\left(\mathbf{r}_{i}^{\mathrm{A}}, \mathbf{r}_{j}^{\mathrm{V}}\right)\right\}=\prod_{i, j}\left(1+h_{i j}^{\mathrm{AV}}\right) \\
& =1+\sum_{i, j} h_{i j}^{\mathrm{AV}}+\sum_{i, j, k, l} h_{i j}^{\mathrm{AV}} h_{k l}^{\mathrm{AV}}+\ldots
\end{aligned}
$$

Then, the density expansion of the mixed term of Eq. (D2), $F^{\mathrm{mix}} \equiv F^{\mathrm{AV}}-F^{\mathrm{A}}-F^{\mathrm{V}}$, takes the form

$$
\begin{aligned}
F^{\mathrm{mix}} & \left(N_{\mathrm{A}}, N_{\mathrm{V}}, T, V\right) \equiv-k T \ln \frac{Q^{\mathrm{AV}}}{Q^{\mathrm{A}} Q^{\mathrm{V}}} \\
= & -k T \ln \left[1+\frac{1}{Q^{\mathrm{A}} Q^{\mathrm{V}}} \int_{V}\left(\frac{\mathrm{d} \mathbf{r}^{\mathrm{A}}}{V}\right)^{N_{\mathrm{A}}}\left(\frac{\mathrm{d} \mathbf{r}^{\mathrm{V}}}{V}\right)^{N_{\mathrm{V}}}\right. \\
& \times\left\{\sum_{i, j} h_{i j}^{\mathrm{AV}}+\sum_{i, j, k, l} h_{i j}^{\mathrm{AV}} h_{k l}^{\mathrm{AV}}+\ldots\right\} \\
& \left.\exp \left\{-\beta \sum_{i<j}^{N_{\mathrm{A}}} u_{\mathrm{AA}}\left(\mathbf{r}_{i}^{\mathrm{A}}, \mathbf{r}_{j}^{\mathrm{A}}\right)-\beta \sum_{i<j}^{N_{\mathrm{V}}} u_{\mathrm{VV}}\left(\mathbf{r}_{i}^{\mathrm{V}}, \mathbf{r}_{j}^{\mathrm{V}}\right)\right\}\right]
\end{aligned}
$$

In the lowest order in density of this series, the exponentials of Eqs. (D4), (D5) and (D8) reduce to unity, and the remaining volume integrals of

$$
F^{\mathrm{mix}}\left(N_{\mathrm{A}}, N_{\mathrm{V}}, T, V\right) \approx-k T \int_{V}\left(\frac{\mathrm{d} \mathbf{r}^{\mathrm{A}}}{V}\right)^{N_{\mathrm{A}}}\left(\frac{\mathrm{d} \mathbf{r}^{\mathrm{V}}}{V}\right)^{N_{\mathrm{V}}}\left\{\sum_{i, j} h_{i j}^{\mathrm{AV}}\right\}
$$

can be carried out except for the particular two arguments of $h_{i j}^{\mathrm{AV}}$. Each term of the sum (D9) provides the same integral; their sum is

$$
F^{\mathrm{mix}}\left(N_{\mathrm{A}}, N_{\mathrm{V}}, T, V\right) \approx-\frac{N_{\mathrm{A}} N_{\mathrm{V}} k T}{V^{2}} \int_{V} \mathrm{~d} \mathbf{r}_{1}^{\mathrm{A}} \mathrm{d} \mathbf{r}_{2}^{\mathrm{V}} h_{12}^{\mathrm{AV}}\left(\mathbf{r}_{1}^{\mathrm{A}}, \mathbf{r}_{2}^{\mathrm{V}}\right)
$$

Computed from Eq. (D2), the density expansion of pressure, $P=-\left(\partial F^{\mathrm{AV}} / \partial V\right)_{N_{\mathrm{A}}, N_{\mathrm{V}}, T}$ can be written in the form (Guggenheim, 1967; Hyland and Wexler, 1983b),

$$
\begin{aligned}
& \frac{P}{k T}=\frac{N_{\mathrm{A}}}{V}\left\{1+\frac{N_{\mathrm{A}}}{V} B_{\mathrm{AA}}(T)+\left(\frac{N_{\mathrm{A}}}{V}\right)^{2} C_{\mathrm{AAA}}(T)+\ldots\right\} \\
& +\frac{N_{\mathrm{V}}}{V}\left\{1+\frac{N_{\mathrm{V}}}{V} B_{\mathrm{WW}}(T)+\left(\frac{N_{\mathrm{V}}}{V}\right)^{2} C_{\mathrm{WWW}}(T)+\ldots\right\} \\
& +\frac{N_{\mathrm{A}}}{V} \frac{N_{\mathrm{V}}}{V}\left\{2 B_{\mathrm{AW}}(T)+3 \frac{N_{\mathrm{A}}}{V} C_{\mathrm{AAW}}(T)+3 \frac{N_{\mathrm{V}}}{V} C_{\mathrm{AWW}}(T)+\ldots\right\}
\end{aligned}
$$

which defines the virial coefficients $B$ and $C$ as functions of the temperature. The 2 nd air-air virial coefficient follows to be a volume integral over the binary air-air correlation function (Reif, 1965; Kammer and Schwabe, 1971; Landau and Lifschitz, 1987), in the form

$B_{\mathrm{AA}}(T)=-\frac{1}{2 V} \int_{V} \mathrm{~d} \mathbf{r}_{1}^{\mathrm{A}} \mathrm{d} \mathbf{r}_{2}^{\mathrm{A}} h_{12}^{\mathrm{AA}}\left(\mathbf{r}_{1}^{\mathrm{A}}, \mathbf{r}_{2}^{\mathrm{A}}\right)$,

and similar for $B_{\mathrm{WW}}$. The term (D10) is proportional to the 2nd cross-virial coefficient $B_{\mathrm{AW}}(T)$ of Harvey and Huang (2007) which is denoted here as $B_{\mathrm{AW}}(T)$, in the corresponding form

$B_{\mathrm{AW}}(T)=-\frac{1}{2 V} \int_{V} \mathrm{~d} \mathbf{r}_{1}^{\mathrm{A}} \mathrm{d} \mathbf{r}_{2}^{\mathrm{V}} h_{12}^{\mathrm{AV}}\left(\mathbf{r}_{1}^{\mathrm{A}}, \mathbf{r}_{2}^{\mathrm{V}}\right)$.

We conclude from this statistical consideration that the mixed Helmholtz function (D8), $F^{\text {mix }}$, yields the correct 2 nd cross-virial coefficient $B_{\mathrm{AW}}$ of Eq. (D11), regardless whether the Helmholtz functions $F^{\mathrm{A}}$ and $F^{\mathrm{V}}$ are used in their compact forms (D2) of Lemmon et al. (2000) and IAPWS-95, or approximated in the form of their virial expansions (D11). Without formal proof we assume that the same conclusion also holds for the higher virial coefficients since the responsible cross-correlation function $h^{\mathrm{AV}}$ appears in each term of the cluster expansion (D8) of $F^{\text {mix }}$ but in none of $F^{\mathrm{A}}$ or $F^{\mathrm{V}}$. In other words, the three terms $F^{\mathrm{A}}, F^{\mathrm{V}}$ and $F^{\text {mix }}$ of the statistically exact formula (D2) possess independent cluster expansions and mutual cancellations of their higher correlations are not possible. Thus, the best approximation for $F^{\mathrm{AV}}$ is obtained from the combination of the best approximations available separately for $F^{\mathrm{A}}, F^{\mathrm{V}}$ and $F^{\text {mix }}$.

Converting Eqs. (D2) and (D11) from particle numbers to mass densities, the specific Helmholtz energy $f^{\mathrm{AV}}\left(T, \rho^{\mathrm{AV}}\right)$ for humid air is Eq. (5.2), up to the 3rd virial coefficients in air-water interaction,

$$
\begin{gathered}
f^{\mathrm{AV}}\left(A, T, \rho^{\mathrm{AV}}\right)=\frac{F^{\mathrm{AV}}}{m^{\mathrm{AV}}}=(1-A) f^{\mathrm{V}}\left(T,(1-A) \rho^{\mathrm{AV}}\right) \\
+A f^{\mathrm{A}}\left(T, A \rho^{\mathrm{AV}}\right)+f^{\operatorname{mix}}\left(A, T, \rho^{\mathrm{AV}}\right)
\end{gathered}
$$

with the cross-virial part of the Helmholtz function

$$
\begin{aligned}
& f^{\operatorname{mix}}\left(A, T, \rho^{\mathrm{AV}}\right)=\frac{2 A(1-A) \rho^{\mathrm{AV}} R T}{M^{\mathrm{A}} M^{\mathrm{W}}} \\
& \left\{B_{\mathrm{AW}}(T)+\frac{3}{4} \rho^{\mathrm{AV}}\left[\frac{A}{M^{\mathrm{A}}} C_{\mathrm{AAW}}(T)+\frac{(1-A)}{M^{\mathrm{W}}} C_{\mathrm{AWW}}(T)\right]\right\} .
\end{aligned}
$$


Here, $A$ is the mass fraction of air in humid air, $f^{\mathrm{A}}\left(T, \rho^{\mathrm{A}}\right)$ is the Helmholtz function of dry air, Eq. (H1), and $f^{\mathrm{V}}\left(T, \rho^{\mathrm{V}}\right)$ is the Helmholtz function of vapour, Eq. (C1). The second virial coefficient, $B_{\mathrm{AW}}(T)$, with $\tau=T /(100 \mathrm{~K})$,

$B_{\mathrm{AW}}=b^{*} \sum_{i=1}^{3} c_{i} \tau^{d_{i}}$

is valid between 100 and $2000 \mathrm{~K}$ (Harvey and Huang, 2007). The reducing factor is $b^{*}=10^{-6} \mathrm{~m}^{3} \mathrm{~mol}^{-1}$, the coefficients are given in Table D1.

The third cross-virial coefficients $C_{\mathrm{AAW}}(T)$ and $C_{\mathrm{AWW}}(T)$ are (Hyland and Wexler, 1983b)

$C_{\mathrm{AAW}}=c^{*} \sum_{i=0}^{4} a_{i} \tau^{i}$

$C_{\mathrm{AWW}}=-c^{*} \exp \left\{\sum_{i=0}^{3} b_{i} \tau^{i}\right\}$.

They are reported in IPTS-48 or IPTS-68, but their conversion to ITS-90 is not necessary because of their large uncertainties of $100 \%$ and $200 \%$. The range of validity is -80 to $220^{\circ} \mathrm{C}$ for $C_{\mathrm{AAW}}$ and -100 to $200^{\circ} \mathrm{C}$ for $C_{\mathrm{AWW}}$ (Hyland and Wexler, 1983b). The reducing constant is $c^{*}=10^{-6} \mathrm{~m}^{6} \mathrm{~mol}^{-2}$, the reduced inverse temperature is $\tau=$ $1 \mathrm{~K} / T$. The coefficients of Eqs. (D17) and (D18) are given in Table D1. Numerical check values are available from Tables I1-I3, Appendix I.

The formula (D14) is valid for $T$ between 193 and $473 \mathrm{~K}$, and for $A$ between 0 or saturation, and 1 . At lower density of at least one of the air or the vapour component, the third virial coefficients can be neglected and the validity range in temperature extends to $100-1273 \mathrm{~K}$.

A comparison of the humid-air formulation of this paper with experimental data is shown in Fig. D1. The incorporation of air-water virial coefficients in the form of a truncated density expansion $F^{\text {mix }}$, Eq. (D8), into the humid-air formulation significantly improves the agreement with measurements at the saturation state. This conclusion regarding the virial coefficients is consistent with the results described by Herrmann et al. (2009a, b, c, 2010). The deviations of these models and of the model of Hyland and Wexler (1983a, b) from the experimental data used in Fig. D1 are within $\pm 2 \%$ in their range of validity up to $10 \mathrm{MPa}\left(\sim 100 \mathrm{~kg} \mathrm{~m}^{-3}\right)$, whereas the mean deviation is not higher than $\pm 0.5 \%$.

The effect of air dissolved in the liquid phase is neglected in this paper. To estimate the related error of the saturation pressure and thus of the relative humidity, we use data for oxygen and nitrogen solubilities in water and seawater taken from the literature (Weiss, 1970; Battino et al., 1984; Sun et al., 2004). For nitrogen in pure water at $0^{\circ} \mathrm{C}$, the solubility is about $1 \mathrm{mmol} \mathrm{kg}^{-1}$ at $0.1 \mathrm{MPa}$ and $50 \mathrm{mmol} \mathrm{kg}^{-1}$ at $5 \mathrm{MPa}$. The related values for oxygen are estimated to be about half

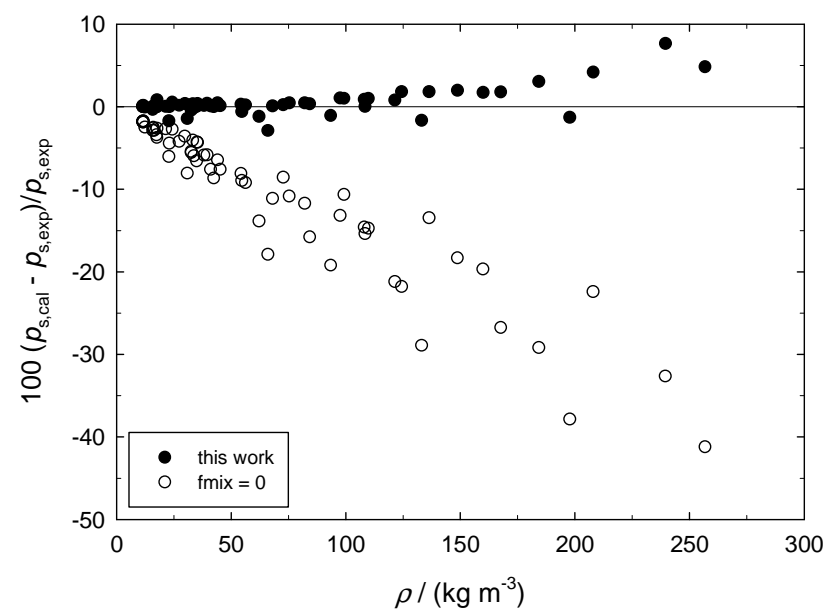

Fig. D1. Deviations of values for the saturation partial pressure of water calculated from different models to experimental data given by Pollitzer and Strebel (1924) at $323 \mathrm{~K}$ and Webster (1950) at 273 and 288 K, Hyland and Wexler (1973) at 303, 313, and $323 \mathrm{~K}$, and Hyland (1975) at $343 \mathrm{~K}$, as well as Wylie and Fisher (1996) at $293 \mathrm{~K}, 323 \mathrm{~K}$ and $348 \mathrm{~K}$, plotted over the total density. Considered models: "this work", calculated from Eq. (7.5), "fmix=0" - this work without the cross-virial coefficients $B$ and $C$, i.e. neglecting $F^{\mathrm{mix}}=0$, Eq. (D8). This result is consistent with earlier studies by Herrmann et al. (2009a, b, c, 2010); the virial model of Hyland and Wexler $(1983 \mathrm{a}, \mathrm{b})$ is included in the latter studies.

as large. At higher temperatures or when sea salt is added, the solubility decreases. From Raoult's law, $\Delta P^{\mathrm{V}} / P^{\mathrm{V}}=-x$, the vapour pressure lowering $\Delta P^{\mathrm{V}}$ of water or seawater can be estimated from the neglected mole fraction $x$ of the gas in the solution (Landau and Lifshitz, 1987). The humid-air formulation of this paper describes experimental data of the saturation vapour pressure within about $0.5 \%$ at $0.1 \mathrm{MPa}$ and $1 \%$ at $5 \mathrm{MPa}$ (humid-air density $\sim 50 \mathrm{~kg} \mathrm{~m}^{-3}$ ), Fig. D1. Thus we may consider the effect of dissolved air as insignificant if the mole fraction $x$ of dissolved gas is less than 0.005 and 0.01 , respectively. Since $1 \mathrm{~kg}$ of water contains about 50 moles of water, the related air molalities must be less than $250 \mathrm{mmol} \mathrm{kg}^{-1}$ and $500 \mathrm{mmol} \mathrm{kg}^{-1}$ to be considered insignificant. The estimated molalities of nitrogen and oxygen are at least a factor of 10 smaller than this tolerance limit, with the largest values corresponding to nitrogen at $5 \mathrm{MPa}$ and $0{ }^{\circ} \mathrm{C}$. The errors incurred by neglecting their effect are therefore insignificant for the vapour pressure lowering of water or seawater at the given uncertainty.

\section{Appendix E}

\section{Thermodynamic potential of seawater}

The Gibbs potential $g\left(S_{\mathrm{A}}, T, P\right)$ of seawater (IAPWS, 2008; Feistel, 2008a) is a function of absolute salinity $S_{\mathrm{A}}$, absolute 
temperature $T$ and absolute pressure $P$. The temperatures are based on the temperature scale ITS-90, the salinities on the Reference-Composition Salinity Scale (Millero et al., 2008). This equation of state for seawater is given as a sum of a pure-water part, $g^{\mathrm{W}}(T, P)$, and a saline part, $g^{\mathrm{S}}\left(S_{\mathrm{A}}, T, P\right)$, defined such that $g^{\mathrm{S}}(0, T, P)=0$,

$g\left(S_{\mathrm{A}}, T, P\right)=g^{\mathrm{W}}(T, P)+g^{\mathrm{S}}\left(S_{\mathrm{A}}, T, P\right)$.

The water part is computed from IAPWS-95, Eq. (C1). Reduced by $g^{*}=1 \mathrm{~J} \mathrm{~kg}^{-1}$, the saline part is the dimensionless polynomial-like function,

$g^{\mathrm{S}}\left(S_{\mathrm{A}}, T, P\right) / g^{*}=\sum_{k=0}^{5} \sum_{j=0}^{6}\left(g_{1 j k} \xi^{2} \ln \xi+\sum_{i=2}^{7} g_{i j k} \xi^{i}\right) \tau^{j} \pi^{k}$,

with the reduced temperature $\tau=\left(T-T_{0}\right) / T^{*}$, the reduced pressure $\pi=\left(P-P_{0}\right) / P^{*}$, and the square root of the reduced salinity, $\xi=\sqrt{S_{\mathrm{A}} / S^{*}}$. The reduced quantities $\tau, \pi$ and $\xi$ vary from 0 to 1 in the oceanographic standard range. The constants $T_{0}, P_{0}, T^{*}, P^{*}$ and $S^{*}$ are given in Table E1.

The coefficients of Eq. (E2) are given in Table E2. Two of these parameters $\left(g_{200}\right.$ and $\left.g_{210}\right)$ are arbitrary and are determined by the reference-state conditions (Feistel et al., 2008b).

Uncertainty estimates are available from IAPWS (2008) and Feistel (2008a). The range of validity given in three regions of the $S_{\mathrm{A}}-T-P$ space is

(a) $0 \leq S_{\mathrm{A}} \leq 42 \mathrm{~g} / \mathrm{kg}, T_{\mathrm{f}} \leq T \leq 313 \mathrm{~K}, 101325 \mathrm{~Pa} \leq P \leq 100 \mathrm{MPa}$,

(b) $0 \leq S_{\mathrm{A}} \leq 50 \mathrm{~g} / \mathrm{kg}, T_{\mathrm{f}} \leq T \leq 313 \mathrm{~K}, P^{\text {vap }} \leq P \leq 101325 \mathrm{~Pa}$,

(c) $0 \leq S_{\mathrm{A}} \leq 120 \mathrm{~g} / \mathrm{kg}, T_{\mathrm{f}} \leq T \leq 353 \mathrm{~K}, P=101325 \mathrm{~Pa}$.

$T_{\mathrm{f}}$ is the freezing temperature, $P^{\text {vap }}$ the vapour pressure of seawater.

Region (c) does not include pressure derivatives of $g$. Density values in (c) are reasonable. Density derivatives may be invalid inside a triangle given by the conditions $T / \mathrm{K}+450 S_{\mathrm{A}} /\left(\mathrm{kg} \mathrm{kg}^{-1}\right)>362, S_{\mathrm{A}} \leq 0.12 \mathrm{~kg} \mathrm{~kg}^{-1}$, and $T \leq$ $353 \mathrm{~K}$. In this triangle, the precipitation of carbonate must be considered, too (Marion et al., 2009). Recent new measurements of seawater densities are expected to improve the Gibbs function for hot concentrates (Millero and Huang, 2009; Safarov et al., 2009; Feistel, 2010).

\section{Appendix F}

\section{Thermodynamic potential of ice Ih}

The Gibbs potential $g^{\mathrm{Ih}}(T, P)$ of ice Ih (IAPWS, 2009b; Feistel and Wagner, 2005, 2006) is a function of absolute temperature $T$ and absolute pressure $P$. This equation of state is given as a function of temperature, with two of its coefficients being polynomial functions of pressure,

$$
\begin{aligned}
& g^{\mathrm{Ih}}(T, P)=g_{0}-s_{0} T_{\mathrm{t}} \cdot \tau+T_{\mathrm{t}} \operatorname{Re} \sum_{k=1}^{2} r_{k} \\
& {\left[\left(t_{k}-\tau\right) \ln \left(t_{k}-\tau\right)+\left(t_{k}+\tau\right) \ln \left(t_{k}+\tau\right)-2 t_{k} \ln t_{k}-\frac{\tau^{2}}{t_{k}}\right]} \\
& g_{0}(P)=\sum_{k=0}^{4} g_{0 k} \cdot\left(\pi-\pi_{0}\right)^{k}, r_{2}(P)=\sum_{k=0}^{2} r_{2 k} \cdot\left(\pi-\pi_{0}\right)^{k}
\end{aligned}
$$

with the reduced temperature $\tau=T / T_{\mathrm{t}}$, the reduced pressure $\pi=P / P_{\mathrm{t}}$, and the reduced normal pressure $\pi_{0}=P_{0} / P_{\mathrm{t}}$, where $T_{\mathrm{t}}=273.16 \mathrm{~K}, P_{\mathrm{t}}=611.657 \mathrm{~Pa}$ is the experimental triple point pressure and $P_{0}=101325 \mathrm{~Pa}$ the normal pressure. The real constants $g_{00}$ to $g_{04}$ and $s_{0}$ as well as the complex constants $t_{1}, r_{1}, t_{2}$, and $r_{20}$ to $r_{22}$ are listed in Table F1.

Uncertainty estimates are available from Feistel and Wagner (2006). Note that the absolute zero-point entropy computed from Table F1 is computed from arbitrary referencestate conditions (Feistel et al., 2008) and is not equal to the statistical residual entropy of ice (Feistel and Wagner, 2005, 2006). The range of validity is given in two $T-P$ regions as

(a) $0<P \leq 101325 \mathrm{~Pa}, 0 \leq T \leq 273.16 \mathrm{~K}$,

(b) $101325 \mathrm{~Pa} \leq P \leq 200 \mathrm{MPa}, 238 \mathrm{~K} \leq T \leq 273.16 \mathrm{~K}$.

The equation of state provides reasonable values also for the region $101325 \mathrm{~Pa} \leq P \leq 200 \mathrm{MPa}, 0 \leq T \leq 238 \mathrm{~K}$, but no uncertainty estimates are available.

\section{Appendix G}

\section{Relations between properties and thermodynamic potentials}

The various partial derivatives of different thermodynamic potentials can be converted into each other by algebraic combinations. Such relations for the Helmholtz energy, the Gibbs energy and the enthalpy are summarized here in Tables G1, G2 and G3. The Jacobi method developed by Shaw (1935) is the mathematically most elegant way of transforming the various partial derivatives of different potential functions into each other, exploiting the convenient formal calculus of functional determinants (Margenau and Murphy, 1943; Guggenheim, 1967; Landau and Lifschitz, 1987).

\section{Appendix H}

\section{Approximate equations for humid air in the ideal-gas limit}

In this section we simplify the equations derived in the previous sections for the ideal-gas approximation which may be 
Table E1. Special constants and values used in Eq. (E2).

\begin{tabular}{llll}
\hline Quantity & Symbol & Value & Unit \\
\hline Normal pressure & $P_{0}$ & 101325 & $\mathrm{~Pa}$ \\
Reducing pressure & $P^{*}$ & 100 & $\mathrm{MPa}$ \\
Celsius zero point & $T_{0}$ & 273.15 & $\mathrm{~K}$ \\
Reducing temperature & $T^{*}$ & 40 & $\mathrm{~K}$ \\
Normal salinity & $S_{\mathrm{n}}$ & 35.16504 & $\mathrm{~g} \mathrm{~kg}^{-1}$ \\
Reducing salinity & & & \\
$S^{*}=S_{\mathrm{n}} \times 40 / 35$ & $S^{*}$ & $40.188617 \ldots$ & $\mathrm{g} \mathrm{kg}^{-1}$ \\
\hline
\end{tabular}

a Normal salinity represents the salinity of KCl-normalized seawater (Millero et al., 2008).

Table E2. Coefficients of the saline part of the Gibbs function (E2). Coefficients not contained in this table have the value $g_{i j k}=0$.

\begin{tabular}{|c|c|c|c|c|c|c|c|}
\hline$i$ & $j$ & $k$ & $g_{i j k}$ & $i$ & $j$ & $k$ & $g_{i j k}$ \\
\hline 1 & 0 & 0 & $+0.581281456626732 \times 10^{4}$ & 2 & 2 & 1 & $-0.860764303783977 \times 10^{3}$ \\
\hline 2 & 0 & 0 & $+0.141627648484197 \times 10^{4}$ & 3 & 2 & 1 & $+0.383058066002476 \times 10^{3}$ \\
\hline 3 & 0 & 0 & $-0.243214662381794 \times 10^{4}$ & 2 & 3 & 1 & $+0.694244814133268 \times 10^{3}$ \\
\hline 4 & 0 & 0 & $+0.202580115603697 \times 10^{4}$ & 3 & 3 & 1 & $-0.460319931801257 \times 10^{3}$ \\
\hline 5 & 0 & 0 & $-0.109166841042967 \times 10^{4}$ & 2 & 4 & 1 & $-0.297728741987187 \times 10^{3}$ \\
\hline 6 & 0 & 0 & $+0.374601237877840 \times 10^{3}$ & 3 & 4 & 1 & $+0.234565187611355 \times 10^{3}$ \\
\hline 7 & 0 & 0 & $-0.485891069025409 \times 10^{2}$ & 2 & 0 & 2 & $+0.384794152978599 \times 10^{3}$ \\
\hline 1 & 1 & 0 & $+0.851226734946706 \times 10^{3}$ & 3 & 0 & 2 & $-0.522940909281335 \times 10^{2}$ \\
\hline 2 & 1 & 0 & $+0.168072408311545 \times 10^{3}$ & 4 & 0 & 2 & $-0.408193978912261 \times 10$ \\
\hline 3 & 1 & 0 & $-0.493407510141682 \times 10^{3}$ & 2 & 1 & 2 & $-0.343956902961561 \times 10^{3}$ \\
\hline 4 & 1 & 0 & $+0.543835333000098 \times 10^{3}$ & 3 & 1 & 2 & $+0.831923927801819 \times 10^{2}$ \\
\hline 5 & 1 & 0 & $-0.196028306689776 \times 10^{3}$ & 2 & 2 & 2 & $+0.337409530269367 \times 10^{3}$ \\
\hline 6 & 1 & 0 & $+0.367571622995805 \times 10^{2}$ & 3 & 2 & 2 & $-0.541917262517112 \times 10^{2}$ \\
\hline 2 & 2 & 0 & $+0.880031352997204 \times 10^{3}$ & 2 & 3 & 2 & $-0.204889641964903 \times 10^{3}$ \\
\hline 3 & 2 & 0 & $-0.430664675978042 \times 10^{2}$ & 2 & 4 & 2 & $+0.747261411387560 \times 10^{2}$ \\
\hline 4 & 2 & 0 & $-0.685572509204491 \times 10^{2}$ & 2 & 0 & 3 & $-0.965324320107458 \times 10^{2}$ \\
\hline 2 & 3 & 0 & $-0.225267649263401 \times 10^{3}$ & 3 & 0 & 3 & $+0.680444942726459 \times 10^{2}$ \\
\hline 3 & 3 & 0 & $-0.100227370861875 \times 10^{2}$ & 4 & 0 & 3 & $-0.301755111971161 \times 10^{2}$ \\
\hline 4 & 3 & 0 & $+0.493667694856254 \times 10^{2}$ & 2 & 1 & 3 & $+0.124687671116248 \times 10^{3}$ \\
\hline 2 & 4 & 0 & $+0.914260447751259 \times 10^{2}$ & 3 & 1 & 3 & $-0.294830643494290 \times 10^{2}$ \\
\hline 3 & 4 & 0 & +0.875600661808945 & 2 & 2 & 3 & $-0.178314556207638 \times 10^{3}$ \\
\hline 4 & 4 & 0 & $-0.171397577419788 \times 10^{2}$ & 3 & 2 & 3 & $+0.256398487389914 \times 10^{2}$ \\
\hline 2 & 5 & 0 & $-0.216603240875311 \times 10^{2}$ & 2 & 3 & 3 & $+0.113561697840594 \times 10^{3}$ \\
\hline 4 & 5 & 0 & $+0.249697009569508 \times 10$ & 2 & 4 & 3 & $-0.364872919001588 \times 10^{2}$ \\
\hline 2 & 6 & 0 & $+0.213016970847183 \times 10$ & 2 & 0 & 4 & $+0.158408172766824 \times 10^{2}$ \\
\hline 2 & 0 & 1 & $-0.331049154044839 \times 10^{4}$ & 3 & 0 & 4 & $-0.341251932441282 \times 10$ \\
\hline 3 & 0 & 1 & $+0.199459603073901 \times 10^{3}$ & 2 & 1 & & $-0.316569643860730 \times 10^{2}$ \\
\hline 4 & 0 & 1 & $-0.547919133532887 \times 10^{2}$ & 2 & 2 & & $+0.442040358308000 \times 10^{2}$ \\
\hline 5 & 0 & 1 & $+0.360284195611086 \times 10^{2}$ & 2 & 3 & 4 & $-0.111282734326413 \times 10^{2}$ \\
\hline 2 & 1 & 1 & $+0.729116529735046 \times 10^{3}$ & 2 & 0 & 5 & $-0.262480156590992 \times 10$ \\
\hline 3 & 1 & 1 & $-0.175292041186547 \times 10^{3}$ & 2 & 1 & 5 & $+0.704658803315449 \times 10$ \\
\hline 4 & 1 & 1 & $-0.226683558512829 \times 10^{2}$ & 2 & 2 & 5 & $-0.792001547211682 \times 10$ \\
\hline
\end{tabular}

Ocean Sci., 6, 91-141, 2010

www.ocean-sci.net/6/91/2010/ 
Table F1. Coefficients of the Gibbs potential (F1).

\begin{tabular}{llll}
\hline Coefficient & Real part & Imaginary part & Unit \\
\hline$g_{00}$ & $-0.632020233335886 \times 10^{6}$ & & $\mathrm{~J} \mathrm{~kg}^{-1}$ \\
$g_{01}$ & +0.655022213658955 & & $\mathrm{~J} \mathrm{~kg}^{-1}$ \\
$g_{02}$ & $-0.189369929326131 \times 10^{-7}$ & & $\mathrm{~J} \mathrm{~kg}^{-1}$ \\
$g_{03}$ & $+0.339746123271053 \times 10^{-14}$ & & $\mathrm{~J} \mathrm{~kg}^{-1}$ \\
$g_{04}$ & $-0.556464869058991 \times 10^{-21}$ & & $\mathrm{~J} \mathrm{~kg}^{-1}$ \\
$s_{0}$ & $-0.332733756492168 \times 10^{4}$ & & $\mathrm{~J} \mathrm{~kg}^{-1} \mathrm{~K}^{-1}$ \\
$t_{1}$ & $+0.368017112855051 \times 10^{-1}$ & $+0.510878114959572 \times 10^{-1}$ & \\
$r_{1}$ & $+0.447050716285388 \times 10^{2}$ & $+0.656876847463481 \times 10^{2}$ & $\mathrm{~J} \mathrm{~kg}^{-1} \mathrm{~K}^{-1}$ \\
$t_{2}$ & +0.337315741065416 & +0.335449415919309 & \\
$r_{20}$ & $-0.725974574329220 \times 10^{2}$ & $-0.781008427112870 \times 10^{2}$ & $\mathrm{~J} \mathrm{~kg}^{-1} \mathrm{~K}^{-1}$ \\
$r_{21}$ & $-0.557107698030123 \times 10^{-4}$ & $+0.464578634580806 \times 10^{-4}$ & $\mathrm{~J} \mathrm{~kg}^{-1} \mathrm{~K}^{-1}$ \\
$r_{22}$ & $+0.234801409215913 \times 10^{-10}$ & $-0.285651142904972 \times 10^{-10}$ & $\mathrm{~J} \mathrm{~kg}^{-1} \mathrm{~K}^{-1}$ \\
\hline
\end{tabular}

Table G1. The partial derivatives of the Helmholtz function, $f(A, T, \rho)$, expressed in terms of thermodynamic coefficients. $\kappa_{T}$ : isothermal compressibility, $\alpha$ : thermal expansion coefficient, $c_{v}$ : specific isochoric heat capacity.

\begin{tabular}{lll}
\hline $\begin{array}{l}\text { Derivative of } \\
f(A, T, \rho)\end{array}$ & Property & Unit \\
\hline$f$ & $u-T s=g-P / \rho$ & $\mathrm{Jkg}^{-1}$ \\
$f_{\rho}$ & $P / \rho^{2}$ & $\mathrm{Jm}^{3} \mathrm{~kg}^{-2}$ \\
$f_{T}$ & $-s$ & $\mathrm{Jkg}^{-1} \mathrm{~K}^{-1}$ \\
$f_{\rho \rho}$ & $\left(1 / \kappa_{T}-2 P\right) / \rho^{3}$ & $\mathrm{Jm}^{6} \mathrm{~kg}^{-3}$ \\
$f_{\rho T}$ & $\alpha /\left(\rho^{2} \kappa_{T}\right)$ & $\mathrm{Jm}^{3} \mathrm{~kg}^{-2} \mathrm{~K}^{-1}$ \\
$f_{T T}$ & $-c_{v} / T$ & $\mathrm{Jkg}^{-1} \mathrm{~K}^{-2}$ \\
\hline
\end{tabular}

Table G2. Partial derivatives of the Gibbs function, $g$, expressed as partial derivatives of the Helmholtz function, $f$. Subscripts indicate partial derivatives with respect to the respective variables.

\begin{tabular}{|c|c|c|c|}
\hline $\begin{array}{l}\text { Derivative of } \\
g(A, T, P)\end{array}$ & Property & $\begin{array}{l}\text { Equivalent in } \\
f(A, T, \rho)\end{array}$ & Unit \\
\hline$P$ & $P$ & $\rho^{2} f_{\rho}$ & $\mathrm{Pa}$ \\
\hline$g$ & $f+P v$ & $f+\rho f_{\rho}$ & $\mathrm{Jkg}^{-1}$ \\
\hline$g_{A}$ & $\mu$ & $f_{A}$ & $\mathrm{Jkg}^{-1}$ \\
\hline$g_{P}$ & $v=\rho^{-1}$ & $\rho^{-1}$ & $\mathrm{~m}^{3} \mathrm{~kg}^{-1}$ \\
\hline$g_{T}$ & $-s$ & $f_{T}$ & $\mathrm{Jkg}^{-1} \mathrm{~K}^{-1}$ \\
\hline$g_{A A}$ & & $f_{A A}-\rho f_{A \rho}^{2} /\left(2 f_{\rho}+\rho f_{\rho \rho}\right)$ & $\mathrm{Jkg}^{-1}$ \\
\hline$g_{A T}$ & & $f_{A T}-\rho f_{A \rho} f_{\rho T} /\left(2 f_{\rho}+\rho f_{\rho \rho}\right)$ & $\mathrm{Jkg}^{-1} \mathrm{~K}^{-1}$ \\
\hline$g_{A P}$ & & $f_{A \rho} /\left[\rho\left(2 f_{\rho}+\rho f_{\rho \rho}\right)\right]$ & $\mathrm{m}^{3} \mathrm{~kg}^{-1}$ \\
\hline$g_{P P}$ & $-\kappa_{T} / \rho$ & $-1 /\left[\rho^{3}\left(2 f_{\rho}+\rho f_{\rho \rho}\right)\right]$ & $\mathrm{m}^{3} \mathrm{~kg}^{-1} \mathrm{~Pa}^{-1}$ \\
\hline$g_{T P}$ & $\alpha / \rho$ & $f_{\rho T} /\left[\rho\left(2 f_{\rho}+\rho f_{\rho \rho}\right)\right]$ & $\mathrm{m}^{3} \mathrm{~kg}^{-1} \mathrm{~K}^{-1}$ \\
\hline$g_{T T}$ & $-c_{P} / T$ & $f_{T T}-\rho f_{\rho T}^{2} /\left(2 f_{\rho}+\rho f_{\rho \rho}\right)$ & $\mathrm{Jkg}^{-1} \mathrm{~K}^{-2}$ \\
\hline
\end{tabular}


Table G3. Partial derivatives of the enthalpy potential function, $h$, expressed as partial derivatives of the Gibbs function, $g$. Subscripts indicate partial derivatives with respect to the respective variables. $\Gamma$ : adiabatic lapse rate, $\kappa_{s}$ : isentropic compressibility.

\begin{tabular}{llll}
\hline $\begin{array}{l}\text { Derivative of } \\
h(A, s, P)\end{array}$ & Equivalent in & Property & Unit \\
\hline$s$ & $g(A, T, P)$ & $s$ & $\mathrm{Jkg}^{-1} \mathrm{~K}^{-1}$ \\
$h$ & $-g_{T}$ & $h$ & $\mathrm{Jkg}^{-1}$ \\
$h_{A}$ & $g-T g_{T}$ & $\mu$ & $\mathrm{Jkg}^{-1}$ \\
$h_{S}$ & $g_{A}$ & $T$ & $\mathrm{~K}$ \\
$h_{P}$ & $g_{P}$ & $v$ & $\mathrm{~m}^{3} \mathrm{~kg}^{-1}$ \\
$h_{A A}$ & $\left(g_{A A} g_{T T}-g_{A T}^{2}\right) / g_{T T}$ & $\mathrm{a}$ & $\mathrm{Jkg}^{-1}$ \\
$h_{A s}$ & $-g_{A T} / g_{T T}$ & $\mathrm{a}$ & $\mathrm{K}$ \\
$h_{A P}$ & $\left(g_{A P} g_{T T}-g_{A T} g_{T P}\right) / g_{T T}$ & $\mathrm{a}$ & $\mathrm{m}^{3} \mathrm{~kg}^{-1}$ \\
$h_{S s}$ & $-1 / g_{T T}$ & $T / c_{p}$ & $\mathrm{kgK}^{2} \mathrm{~J}^{-1}$ \\
$h_{S P}$ & $-g_{T P} / g_{T T}$ & $\Gamma$ & $\mathrm{KPa}^{-1}$ \\
$h_{P P}$ & $\left(g_{T T} g_{P P}-g_{T P}^{2}\right) / g_{T T}$ & $-v \kappa_{S}=-v^{2} / c^{2}$ & $\mathrm{~m}^{3} \mathrm{~kg}^{-1} \mathrm{~Pa}^{-1}$ \\
\hline
\end{tabular}

a The quantity $g_{A T}$ appearing here is related to the thermodiffusion coefficient (Landau and Lifschitz, 1974, §58) but has no common name or symbol.

sufficiently accurate for certain application purposes. Most of these relations are well known (Guggenheim, 1967); we repeat the formulas here for easy reference to the same quantities in the more general case of the real gas, and in particular for their adjustable constants chosen consistently with the real-gas formulation, Table H1. This consistency is indispensable for applications such as the fugacity (10.8). The Helmholtz functions (H1) and (H2) are equivalent to the ideal parts (B2) and (C2) except that the temperature dependencies of the heat capacities are not specified here and may be replaced by simpler expressions if this is appropriate.

The ideal-gas part of the Helmholtz function for dry air, Eq. (B2), takes the form

$$
\begin{aligned}
& f^{\mathrm{A}, \mathrm{id}}\left(T, \rho^{\mathrm{A}}\right)=\frac{1}{m^{\mathrm{A}}} \sum_{a} F_{a}^{\mathrm{id}} \\
& =f_{0}^{\mathrm{A}}-\left(T-T_{0}^{\mathrm{A}}\right) s_{0}^{\mathrm{A}}+\int_{T_{0}^{\mathrm{A}}}^{T}\left(1-\frac{T}{T^{\prime}}\right) \cdot c_{V}^{\mathrm{A}, \mathrm{id}}\left(T^{\prime}\right) \mathrm{d} T^{\prime} \\
& \quad+R_{\mathrm{A}}^{\mathrm{L}} T \ln \frac{\rho^{\mathrm{A}}}{\rho_{0}^{\mathrm{A}}} .
\end{aligned}
$$

Here, $R_{\mathrm{A}}^{\mathrm{L}}=R^{\mathrm{L}} / M^{\mathrm{A}, \mathrm{L}}$ is the specific gas constant of air, and $T_{0}^{\mathrm{A}}, f_{0}^{\mathrm{A}}, s_{0}^{\mathrm{A}}, \rho_{0}^{\mathrm{A}}$ are mutually related, freely adjustable constants, Table H1, subject to reference state conditions. The formal temperature $T^{\prime}$ in Eq. (H1) and in the following equations is a dummy integration variable that takes any value from the lower through the upper bound of the integral.
The ideal-gas Helmholtz function of water vapour has the similar form,

$$
\begin{aligned}
& f^{\mathrm{V}, \mathrm{id}}\left(T, \rho^{\mathrm{V}}\right)=f_{0}^{\mathrm{V}}-\left(T-T_{0}^{\mathrm{V}}\right) s_{0}^{\mathrm{V}}+\int_{T_{0}^{\mathrm{V}}}^{T}\left(1-\frac{T}{T^{\prime}}\right) \\
& c_{V}^{\mathrm{V}, \mathrm{id}}\left(T^{\prime}\right) \mathrm{d} T^{\prime}+R_{\mathrm{W}}^{95} T \ln \frac{\rho^{\mathrm{V}}}{\rho_{0}^{\mathrm{V}}}
\end{aligned}
$$

For the Helmholtz function of humid air we obtain from Eq. (5.2) in this limit, Eq. (D1),

$$
\begin{aligned}
& f^{\mathrm{AV}, \mathrm{id}}\left(A, T, \rho^{\mathrm{AV}}\right)= \\
& A\left[f_{0}^{\mathrm{A}}-\left(T-T_{0}^{\mathrm{A}}\right) s_{0}^{\mathrm{A}}+\int_{T_{0}^{\mathrm{A}}}^{T}\left(1-\frac{T}{T^{\prime}}\right) \cdot c_{V}^{\mathrm{A}, \mathrm{id}}\left(T^{\prime}\right) \mathrm{d} T^{\prime}+R_{\mathrm{A}}^{\mathrm{L}} T \ln \frac{A \rho^{\mathrm{AV}}}{\rho_{0}^{\mathrm{A}}}\right] \\
& +(1-A)\left[f_{0}^{\mathrm{V}}-\left(T-T_{0}^{\mathrm{V}}\right) s_{0}^{\mathrm{V}}+\int_{T_{0}^{\mathrm{V}}}^{T}\left(1-\frac{T}{T^{\prime}}\right)\right. \\
& \left.\cdot c_{V}^{\mathrm{V}, \mathrm{id}}\left(T^{\prime}\right) \mathrm{d} T^{\prime}+R_{\mathrm{W}}^{95} T \ln \frac{(1-A) \rho^{\mathrm{AV}}}{\rho_{0}^{\mathrm{V}}}\right] .
\end{aligned}
$$

Having computed the pressure from the density derivative of Eq. (H3), as

$$
\begin{aligned}
P^{\mathrm{AV}, \mathrm{id}} & =\left(\rho^{\mathrm{AV}}\right)^{2}\left(\partial f^{\mathrm{AV}, \mathrm{id}} / \partial \rho^{\mathrm{AV}}\right)_{A, T} \\
& =\rho^{\mathrm{AV}}\left[(1-A) R_{\mathrm{W}}^{95}+A R_{\mathrm{A}}^{\mathrm{L}}\right] T,
\end{aligned}
$$


Table H1. Consistently chosen adjustable constants and related properties of the ideal-gas formulas with respect to the ideal-gas parts of the formulations for dry air, "LJPF", (Lemmon et al., 2000), Eq. (B2), and water vapour, "IAPWS95", Eq. (C3), and their reference states. The densities $\rho_{0}^{\mathrm{A}}$ and $\rho_{0}^{\mathrm{V}}$ satisfy the ideal-gas equations $\rho_{0}^{\mathrm{A}} R_{\mathrm{A}}^{\mathrm{L}} T_{0}=P_{0}$ and $\rho_{0}^{\mathrm{V}} R_{\mathrm{W}}^{95} T_{\mathrm{t}}=P_{\mathrm{t}}$. For comparison, if the latest values $M^{\mathrm{A}}=28.96546 \mathrm{~g} \mathrm{~mol}^{-1}, M^{\mathrm{W}}=18.015268 \mathrm{~g} \mathrm{~mol}^{-1}$ and $R=8.314472 \mathrm{~J} \mathrm{~mol}^{-1} \mathrm{~K}^{-1}$ are used rather than the older values, the related ideal-gas densities at the reference points change to $\rho^{\mathrm{A}, \mathrm{id}}=M_{\mathrm{A}} P_{0} /\left(R T_{0}\right)=1.2922935073108 \mathrm{~kg} \mathrm{~m}^{-3}$ and $\rho^{\mathrm{V} \text {,id }}=$ $M_{\mathrm{W}} P_{\mathrm{t}} /\left(R T_{t}\right)=0.00485171538112533 \mathrm{~kg} \mathrm{~m}^{-3}$.

\begin{tabular}{lllll}
\hline Constant & Value & Constant & Value & Unit \\
\hline$f_{0}^{\mathrm{A}}$ & -78148.1837806036 & $f_{0}^{\mathrm{V}}$ & 2375392.69430482 & $\mathrm{Jkg}^{-1}$ \\
$g_{0}^{\mathrm{A}}$ & 277.859139288958 & $g_{0}^{\mathrm{V}}$ & 2501460.96484282 & $\mathrm{Jkg}^{-1}$ \\
$h_{0}^{\mathrm{A}}$ & 277.859139288958 & $h_{0}^{\mathrm{V}}$ & 2501460.96484282 & $\mathrm{Jkg}^{-1}$ \\
$P_{0}^{\mathrm{A}}$ & 101623.289881139 & $P_{0}^{\mathrm{V}}$ & 253269701789.662 & $\mathrm{~Pa}^{\mathrm{V}}$ \\
$s_{0}^{\mathrm{A}}$ & 0.844000164354527 & $s_{0}^{\mathrm{V}}$ & 9157.23473377111 & $\mathrm{Jkg}^{-1} \mathrm{~K}^{-1}$ \\
$T_{0}^{\mathrm{A}}$ & 273.15 & $T_{0}^{\mathrm{V}}$ & 273.16 & $\mathrm{~V}$ \\
$\rho_{0}^{\mathrm{A}}$ & 1.29198154372646 & $\rho_{0}^{\mathrm{N}}$ & 0.00485177410935868 & $\mathrm{~kg} \mathrm{~m}^{-3}$ \\
$g^{\mathrm{LJPF}, \text { id }}\left(T_{0}, P_{0}\right)$ & 47.3204943955194 & $g^{\text {IAPWS9,id }}\left(T_{\mathrm{t}}, P_{\mathrm{t}}\right)$ & 70.7249659009445 & $\mathrm{Jkg}^{-1}$ \\
$s^{\mathrm{LJPF}, \mathrm{id}}\left(T_{0}, P_{0}\right)$ & 0.844000164354527 & $s^{\text {IAPWS95,id }}\left(T_{\mathrm{t}}, P_{\mathrm{t}}\right)$ & 9157.23473377111 & $\mathrm{Jkg}^{-1} \mathrm{~K}^{-1}$ \\
$c_{P}^{\mathrm{LJPF}, \text { id }}\left(T_{0}, P_{0}\right)$ & 1003.9199246143 & $c_{P}$ IAPWS95,id $\left(T_{\mathrm{t}}, P_{\mathrm{t}}\right)$ & 1859.01849914039 & $\mathrm{Jkg}^{-1} \mathrm{~K}^{-1}$ \\
$c_{V}^{\mathrm{LJPF}, \mathrm{id}}\left(T_{0}, P_{0}\right)$ & 716.802798786396 & $c_{V}^{\text {APWS95,id }}\left(T_{\mathrm{t}}, P_{\mathrm{t}}\right)$ & 1397.50044914039 & $\mathrm{Jkg}^{-1} \mathrm{~K}^{-1}$ \\
$T_{0}$ & 273.15 & $T_{\mathrm{t}}$ & 273.16 & $\mathrm{~K}$ \\
$P_{0}$ & 101325 & $P_{\mathrm{t}}$ & 611.654771007894 & $\mathrm{~Pa}$ \\
\hline
\end{tabular}

we derive the ideal-gas Gibbs function of humid air, $g^{\mathrm{AV}, \mathrm{id}}=$ $f^{\mathrm{AV}, \mathrm{id}}+P^{\mathrm{AV}, \mathrm{id}} / \rho^{\mathrm{AV}}$, Eq. (5.12), as

$$
\begin{aligned}
& g^{\mathrm{AV}, \mathrm{id}}(A, T, P) \\
& =A\left[g_{0}^{\mathrm{A}}+\int_{T_{0}^{\mathrm{A}}}^{T}\left(1-\frac{T}{T^{\prime}}\right) \cdot c_{P}^{\mathrm{A}, \mathrm{id}} \mathrm{d} T^{\prime}+R_{\mathrm{A}}^{\mathrm{L}} T \ln \frac{x_{\mathrm{A}}^{\mathrm{AV}} P}{P_{0}^{\mathrm{A}}}\right] \\
& +(1-A)\left[g_{0}^{\mathrm{V}}+\int_{T_{0}^{\mathrm{V}}}^{T}\left(1-\frac{T}{T^{\prime}}\right) \cdot c_{P}^{\mathrm{V}, \mathrm{id}} \mathrm{d} T^{\prime}+R_{\mathrm{W}}^{95} T \ln \frac{x_{\mathrm{V}}^{\mathrm{AV}} P}{P_{0}^{\mathrm{V}}}\right] .
\end{aligned}
$$

Here, the pressure as an independent variable is written without superscripts, $P \equiv P^{\mathrm{AV}}$,id. The related entropy follows as

$$
\begin{aligned}
& s^{\mathrm{AV}, \mathrm{id}}(A, T, P)=A\left[\int_{T_{0}^{\mathrm{A}}}^{T} \frac{c_{P}^{\mathrm{A}, \mathrm{id}}\left(T^{\prime}\right)}{T^{\prime}} \mathrm{d} T^{\prime}-R_{\mathrm{A}}^{\mathrm{L}} \ln \frac{x_{\mathrm{A}}^{\mathrm{AV}} P}{P_{0}^{\mathrm{A}}}\right] \\
& +(1-A)\left[\int_{T_{0}^{\mathrm{V}}}^{T} \frac{c_{P}^{\mathrm{V}, \mathrm{id}}\left(T^{\prime}\right)}{T^{\prime}} \mathrm{d} T^{\prime}-R_{\mathrm{W}}^{95} \ln \frac{x_{\mathrm{V}}^{\mathrm{AV}} P}{P_{0}^{\mathrm{V}}}\right]
\end{aligned}
$$

with the heat capacities $c_{P}^{\mathrm{A}, \mathrm{id}}(T)=c_{V}^{\mathrm{A}, \mathrm{id}}(T)+R_{\mathrm{A}}^{\mathrm{L}}$, $c_{P}^{\mathrm{V} \text {,id }}(T)=c_{V}^{\mathrm{V} \text {,id }}(T)+R_{\mathrm{W}}^{95}$ and the modified adjustable constants, Table H1,

$$
\begin{aligned}
& P_{0}^{\mathrm{A}}=\rho_{0}^{\mathrm{A}} R_{\mathrm{A}}^{\mathrm{L}} T_{0}^{\mathrm{A}} \exp \left(s_{0}^{\mathrm{A}} / R_{\mathrm{A}}^{\mathrm{L}}\right), \\
& P_{0}^{\mathrm{V}}=\rho_{0}^{\mathrm{V}} R_{\mathrm{W}}^{95} T_{0}^{\mathrm{V}} \exp \left(s_{0}^{\mathrm{V}} / R_{\mathrm{W}}^{95}\right) .
\end{aligned}
$$

$P^{\mathrm{V}}(A, P) \equiv x_{\mathrm{V}}^{\mathrm{AV}} P$ is the partial vapour pressure and $x_{\mathrm{V}}^{\mathrm{AV}}=(1-A) R_{\mathrm{W}}^{95} /\left[(1-A) R_{\mathrm{W}}^{95}+A R_{\mathrm{A}}^{\mathrm{L}}\right]$ is the mole fraction of vapour in humid air, Eq. (5.6), $P^{\mathrm{A}}(A, P) \equiv$ $x_{\mathrm{A}}^{\mathrm{AV}} P$ is the partial air pressure and $x_{\mathrm{A}}^{\mathrm{AV}}=1-x_{\mathrm{V}}^{\mathrm{AV}}=$ $A R_{\mathrm{A}}^{\mathrm{L}} /\left[(1-A) R_{\mathrm{W}}^{95}+A R_{\mathrm{A}}^{\mathrm{L}}\right]$ is the mole fraction of air.

The expression (H6) can be written in the form

$$
\begin{aligned}
& s^{\mathrm{AV}, \text { id }}(A, T, P)=A s^{\mathrm{A}, \mathrm{id}}(T, P)+(1-A) s^{\mathrm{V}, \mathrm{id}}(T, P) \\
& \quad+s^{\mathrm{mix}}(A) .
\end{aligned}
$$

Here, the entropy of mixing is

$s^{\text {mix }}(A)=-A R_{\mathrm{A}}^{\mathrm{L}} \ln x_{\mathrm{A}}^{\mathrm{AV}}-(1-A) R_{\mathrm{W}}^{95} \ln x_{\mathrm{V}}^{\mathrm{AV}} \geq 0$.

The ideal-gas enthalpy of humid air, $h^{\mathrm{AV}, \text { id }}=g^{\mathrm{AV}, \text { id }}+$ $T s^{\mathrm{AV} \text {,id }}$, as a function of temperature does not depend on the pressure:

$$
\begin{gathered}
h^{\mathrm{AV}, \mathrm{id}}(A, T, P)=A\left[h_{0}^{\mathrm{A}}+\int_{T_{0}^{\mathrm{A}}}^{T} c_{P}^{\mathrm{A}, \mathrm{id}}\left(T^{\prime}\right) \mathrm{d} T^{\prime}\right] \\
+(1-A)\left[h_{0}^{\mathrm{V}}+\int_{T_{0}^{\mathrm{V}}}^{T} c_{P}^{\mathrm{V}, \mathrm{id}}\left(T^{\prime}\right) \mathrm{d} T^{\prime}\right] .
\end{gathered}
$$

The partial enthalpy of water, Eq. (6.28), in ideal-gas humid air follows from Eq. (H11) as

$$
h^{\mathrm{AV}, \mathrm{id}}-A\left(\frac{\partial h^{\mathrm{AV}, \mathrm{id}}}{\partial A}\right)_{T, P}=h_{0}^{\mathrm{V}}+\int_{T_{0}^{\mathrm{V}}}^{T} c_{P}^{\mathrm{V}, \mathrm{id}}\left(T^{\prime}\right) \mathrm{d} T^{\prime}
$$

and equals the ideal-gas enthalpy of vapour. 
Enthalpy as a thermodynamic potential, $h^{\mathrm{AV}, \mathrm{id}}(A, s, P)$, expressed in terms of air fraction, entropy and pressure, is useful for the computation of isentropic properties of the atmosphere. To derive this function, temperature must be eliminated from Eqs. (H6) and (H11). This is analytically possible only if the heat capacities of air and vapour are given by simple formulas such as constants, e.g., for applications in narrow temperature intervals.

For the chemical potential of vapour in humid air we get from Eq. (5.15),

$$
\begin{aligned}
& \mu^{\mathrm{V}, \mathrm{id}}(A, T, P)=g_{0}^{\mathrm{V}}+\int_{T_{0}^{\mathrm{V}}}^{T}\left(1-\frac{T}{T^{\prime}}\right) \cdot c_{P}^{\mathrm{V}, \mathrm{id}}\left(T^{\prime}\right) \mathrm{d} T^{\prime} \\
& \quad+R_{\mathrm{W}}^{95} T \ln \frac{x_{\mathrm{V}}^{\mathrm{AV}} P}{P_{0}^{\mathrm{V}}} .
\end{aligned}
$$

The adjustable coefficients $g_{0}^{\mathrm{A}}, g_{0}^{\mathrm{V}}, h_{0}^{\mathrm{A}}$ and $h_{0}^{\mathrm{V}}$ are given in Table H1. The relative chemical potential is

$$
\begin{aligned}
& \mu^{\mathrm{AV}, \mathrm{id}}=\left(\frac{\partial g^{\mathrm{AV}, \mathrm{id}}}{\partial A}\right)_{T, P}=\mu^{\mathrm{A}, \mathrm{id}}-\mu^{\mathrm{V}, \mathrm{id}} \\
& =g_{0}^{\mathrm{A}}+\int_{T_{0}^{\mathrm{A}}}^{T}\left(1-\frac{T}{T^{\prime}}\right) \cdot c_{P}^{\mathrm{A}, \mathrm{id}} \mathrm{d} T^{\prime}+R_{\mathrm{A}}^{\mathrm{L}} T \ln \frac{x_{\mathrm{A}}^{\mathrm{AV}} P}{P_{0}^{\mathrm{A}}} \\
& -g_{0}^{\mathrm{V}}-\int_{T_{0}^{\mathrm{V}}}^{T}\left(1-\frac{T}{T^{\prime}}\right) \cdot c_{P}^{\mathrm{V}, \mathrm{id}} \mathrm{d} T^{\prime}-R_{\mathrm{W}}^{95} T \ln \frac{x_{\mathrm{V}}^{\mathrm{AV} P}}{P_{0}^{\mathrm{V}}}
\end{aligned}
$$

and the second derivative of the Gibbs function (H5), i.e., the chemical coefficient (6.8) is given by

$$
A(1-A)\left(\frac{\partial^{2} g^{\mathrm{AV}, \text { id }}}{\partial A^{2}}\right)_{T, P}=\frac{R_{\mathrm{W}}^{95} R_{\mathrm{A}}^{\mathrm{L}} T}{R_{\mathrm{W}}^{95}(1-A)+A R_{\mathrm{A}}^{\mathrm{L}}} .
$$

To consistently adjust the free coefficients of the idealgas equations of this section to the reference state conditions used for the formulation IAPWS-95, Eq. (C3), and Lemmon et al. (2000), Eq. (B2), we require that the ideal-gas parts of the latter two provide the same values for the entropy, $s^{\text {IAPWS95,id }}\left(T_{\mathrm{t}}, P_{\mathrm{t}}\right)$, and the Gibbs energy, $g^{\text {IAPWS95, id }}\left(T_{\mathrm{t}}, P_{\mathrm{t}}\right)$, of vapour at the IAPWS-95 triple point, $P_{\mathrm{t}}=611.654771007894 \mathrm{~Pa}, T_{\mathrm{t}}=273.16 \mathrm{~K}$, and for the entropy, $s^{\mathrm{LJPF}, \mathrm{id}}\left(T_{0}, P_{0}\right)$, and the Gibbs energy, $g^{\mathrm{LJPF}, \mathrm{id}}\left(T_{0}, P_{0}\right)$, of dry air at the standard ocean surface state, $P_{0}=101325 \mathrm{~Pa}$, $T_{0}=273.15 \mathrm{~K}$.

The approximation used for the ideal-gas heat capacities of air and vapour in Eqs. (H3), (H5), (H6) etc. is restricted to pressure-independent functions of temperature (or constants); the particular choice depends on the required accuracy of the application purpose. Values for $c_{P}$ at the reference states are given in Table H1. For a wider range or a different working point, $c_{P}$ can, for example, be computed from the temperature derivative of Eq. (5.17) at very low pressure $P \rightarrow 0$ and in the limiting cases $A \rightarrow 0$ for vapour and $A \rightarrow 1$ for dry air. In general it is recommended to implement the heat capacity formula derived from the ideal-gas parts of dry air, Eq. (B2), and vapour, Eq. (C2).

For the computation of the adjustable coefficients we use the equations for the Gibbs energy of the pure components, Eq. (H5) at $A=0$ and $A=1$,

$$
g^{\mathrm{A}, \mathrm{id}}(T, P)=g_{0}^{\mathrm{A}}+\int_{T_{0}^{\mathrm{A}}}^{T}\left(1-\frac{T}{T^{\prime}}\right) \cdot c_{P}^{\mathrm{A}, \mathrm{id}}\left(T^{\prime}\right) \mathrm{d} T^{\prime}+R_{\mathrm{A}}^{\mathrm{L}} T \ln \frac{P}{P_{0}^{\mathrm{A}}},
$$

$$
g^{\mathrm{V}, \mathrm{id}}(T, P)=g_{0}^{\mathrm{V}}+\int_{T_{0}^{\mathrm{V}}}^{T}\left(1-\frac{T}{T^{\prime}}\right) \cdot c_{P}^{\mathrm{V}, \mathrm{id}}\left(T^{\prime}\right) \mathrm{d} T^{\prime}+R_{\mathrm{W}}^{95} T \ln \frac{P}{P_{0}^{\mathrm{V}}},
$$

and for their entropies, Eq. (H6) at $A=0$ and $A=1$,

$$
\begin{gathered}
s^{\mathrm{A}, \mathrm{id}}(T, P)=\int_{T_{0}^{\mathrm{A}}}^{T} \frac{c_{P}^{\mathrm{A}, \mathrm{id}}\left(T^{\prime}\right)}{T^{\prime}} \mathrm{d} T^{\prime}-R_{\mathrm{A}}^{\mathrm{L}} \ln \frac{P}{P_{0}^{\mathrm{A}}}, \\
s^{\mathrm{V}, \mathrm{id}}(T, P)=\int_{T_{0}^{\mathrm{V}}}^{T} \frac{c_{P}^{\mathrm{V}, \mathrm{id}}\left(T^{\prime}\right)}{T^{\prime}} \mathrm{d} T^{\prime}-R_{\mathrm{W}}^{95} \ln \frac{P}{P_{0}^{\mathrm{V}}} .
\end{gathered}
$$

We set the adjustable temperatures to $T_{0}^{\mathrm{V}}=273.16 \mathrm{~K}$ and $T_{0}^{\mathrm{A}}=273.15 \mathrm{~K}$ and express the adjustable pressures from Eqs. (H18), (H19) in terms of the absolute entropies at the reference points of the ideal parts of the full equations,

$$
P_{0}^{\mathrm{A}}=P_{0} \exp \left(s^{\mathrm{LJPF}, \mathrm{id}}\left(T_{0}, P_{0}\right) / R_{\mathrm{A}}^{\mathrm{L}}\right)
$$

$$
P_{0}^{\mathrm{V}}=P_{\mathrm{t}} \exp \left(s^{\mathrm{IAPWS} 95, \text { id }}\left(T_{\mathrm{t}}, P_{\mathrm{t}}\right) / R_{\mathrm{W}}^{95}\right) .
$$

Inserting Eqs. (H18), (H19) into Eqs. (H16), (H17), we find for the adjustable Gibbs energies

$g_{0}^{\mathrm{A}}=g^{\mathrm{LJPF}, \mathrm{id}}\left(T_{0}, P_{0}\right)+T_{0} s^{\mathrm{LJPF}, \mathrm{id}}\left(T_{0}, P_{0}\right)$

$g_{0}^{\mathrm{V}}=g^{\mathrm{IAPWS} 95, \text { id }}\left(T_{\mathrm{t}}, P_{\mathrm{t}}\right)+T_{\mathrm{t}} s^{\mathrm{IAPWS} 95, \text { id }}\left(T_{\mathrm{t}}, P_{\mathrm{t}}\right)$.

Choosing the adjustable densities $\rho_{0}^{\mathrm{A}}$ and $\rho_{0}^{\mathrm{V}}$ in Eqs. (H7), (H8), conveniently to satisfy the ideal-gas equations $\rho_{0}^{\mathrm{A}} R_{\mathrm{A}}^{\mathrm{L}} T_{0}^{\mathrm{A}}=P_{0}$ and $\rho_{0}^{\mathrm{V}} R_{\mathrm{W}}^{95} T_{0}^{\mathrm{V}}=P_{\mathrm{t}}$, we get for the adjustable entropies of Eq. (H3),

$$
\begin{aligned}
& s_{0}^{\mathrm{A}}=s^{\mathrm{LJPF}, \mathrm{id}}\left(T_{0}, P_{0}\right) \\
& s_{0}^{\mathrm{V}}=s^{\mathrm{IAPWS} 95, \mathrm{id}}\left(T_{\mathrm{t}}, P_{\mathrm{t}}\right) .
\end{aligned}
$$

Adding up Eqs. (H16) and (H18) at $T=T_{0}^{\mathrm{A}}$, and correspondingly for Eqs. (H17) and (H19) at $T=T_{0}^{\mathrm{V}}$, we get from Eq. (H11) the adjustable enthalpies 
Table I1. Numerical check values for the Helmholtz function $f^{\mathrm{AV}}$ and its derivatives, Eq. (D14), as well as selected properties, Eqs. (5.95.24), for saturated air, $A=A^{\text {sat }}$, with respect to ice at $200 \mathrm{~K}$, and with respect to liquid water at 300 and $400 \mathrm{~K}$. The humid-air density is $\rho=\rho^{\mathrm{AV}}=\rho^{\mathrm{A}}+\rho^{\mathrm{V}}=m^{\mathrm{AV}} / V=\left(m^{\mathrm{A}}+m^{\mathrm{V}}\right) / V$.

\begin{tabular}{|c|c|c|c|c|}
\hline Quantity & Value & Value & Value & Unit \\
\hline$A$ & +0.892224944 & +0.977600624 & +0.825531379 & $\mathrm{~kg} \mathrm{~kg}^{-1}$ \\
\hline$T$ & +200 & +300 & +400 & $\mathrm{~K}$ \\
\hline$\rho=\rho^{\mathrm{AV}}$ & $+0.163445112 \times 10^{-4}$ & $+0.114587678 \times 10$ & $+0.793198757 \times 10$ & $\mathrm{~kg} \mathrm{~m}^{-3}$ \\
\hline$p$ & $+0.100000000 \times 10$ & $+0.100000000 \times 10^{6}$ & $+0.100000000 \times 10^{7}$ & $\mathrm{~Pa}$ \\
\hline$f^{\mathrm{AV}}$ & $-0.682237558 \times 10^{6}$ & $-0.927933016 \times 10^{5}$ & $+0.240393277 \times 10^{5}$ & $\mathrm{~J} \mathrm{~kg}^{-1}$ \\
\hline$\left(\partial f^{A V} / \partial A\right)_{T, \rho}$ & $-0.572842114 \times 10^{6}$ & $-0.264760901 \times 10^{3}$ & $+0.311145187 \times 10^{6}$ & $\mathrm{~J} \mathrm{~kg}^{-1}$ \\
\hline$\left(\partial f^{A V} / \partial T\right)_{A, \rho}$ & $-0.405403634 \times 10^{4}$ & $-0.296780108 \times 10^{3}$ & $-0.106912744 \times 10^{4}$ & $\mathrm{~J} \mathrm{~kg}^{-1} \mathrm{~K}^{-1}$ \\
\hline$\left(\partial f^{\mathrm{AV}} / \partial \rho\right)_{A, T}$ & $+0.374331286 \times 10^{10}$ & $+0.761595138 \times 10^{5}$ & $+0.158941003 \times 10^{5}$ & $\mathrm{~J} \mathrm{~m}^{3} \mathrm{~kg}^{-2}$ \\
\hline$\left(\partial^{2} f^{\mathrm{AV}} / \partial A^{2}\right)_{T, \rho}$ & $+0.920803547 \times 10^{6}$ & $+0.624746061 \times 10^{7}$ & $+0.113770932 \times 10^{7}$ & $\mathrm{~J} \mathrm{~kg}^{-1}$ \\
\hline$\left(\partial^{2} f^{\mathrm{AV}} / \partial A \partial T\right)_{\rho}$ & $+0.915588345 \times 10^{4}$ & $+0.822737763 \times 10^{4}$ & $+0.702643265 \times 10^{4}$ & $\mathrm{~J} \mathrm{~kg}^{-1} \mathrm{~K}^{-1}$ \\
\hline$\left(\partial^{2} f^{\mathrm{AV}} / \partial A \partial \rho\right)_{T}$ & $-0.213404004 \times 10^{10}$ & $-0.449931362 \times 10^{5}$ & $-0.727775043 \times 10^{4}$ & $\mathrm{~J} \mathrm{~m}^{3} \mathrm{~kg}^{-2}$ \\
\hline$\left(\partial^{2} f^{\mathrm{AV}} / \partial T^{2}\right)_{A, \rho}$ & $-0.394095200 \times 10$ & $-0.244799624 \times 10$ & $-0.222492603 \times 10$ & $\mathrm{~J} \mathrm{~kg}^{-1} \mathrm{~K}^{-2}$ \\
\hline$\left(\partial^{2} f^{\mathrm{AV}} / \partial T \partial \rho\right)_{A}$ & $+0.187166127 \times 10^{8}$ & $+0.254574163 \times 10^{3}$ & $+0.414512281 \times 10^{2}$ & $\mathrm{~J} \mathrm{~m}^{3} \mathrm{~kg}^{-2} \mathrm{~K}^{-1}$ \\
\hline$\left(\partial^{2} f^{\mathrm{AV}} / \partial \rho^{2}\right)_{A, T}$ & $-0.229025760 \times 10^{15}$ & $-0.664927281 \times 10^{5}$ & $-0.202004713 \times 10^{4}$ & $\mathrm{~J} \mathrm{~m}^{6} \mathrm{~kg}^{-3}$ \\
\hline$h^{\mathrm{AV}}$ & $+0.189752330 \times 10^{6}$ & $+0.835101493 \times 10^{5}$ & $+0.577762111 \times 10^{6}$ & $\mathrm{~J} \mathrm{~kg}^{-1}$ \\
\hline$g^{\mathrm{AV}}$ & $-0.621054939 \times 10^{6}$ & $-0.552388319 \times 10^{4}$ & $+0.150111134 \times 10^{6}$ & $\mathrm{~J} \mathrm{~kg}^{-1}$ \\
\hline$s^{\mathrm{AV}}$ & $+0.405403634 \times 10^{4}$ & $+0.296780108 \times 10^{3}$ & $+0.106912744 \times 10^{4}$ & $\mathrm{~J} \mathrm{~kg}^{-1} \mathrm{~K}^{-1}$ \\
\hline$\mu^{\mathrm{V}}$ & $-0.109950916 \times 10^{6}$ & $-0.526505277 \times 10^{4}$ & $-0.106748982 \times 10^{6}$ & $\mathrm{~J} \mathrm{~kg}^{-1}$ \\
\hline$c_{P}^{\mathrm{AV}}$ & $+0.109410518 \times 10^{4}$ & $+0.102705097 \times 10^{4}$ & $+0.123576406 \times 10^{4}$ & $\mathrm{~J} \mathrm{~kg}^{-1} \mathrm{~K}^{-1}$ \\
\hline$c^{r}$ & $+0.291425752 \times 10^{3}$ & $+0.349274633 \times 10^{3}$ & $+0.416697519 \times 10^{3}$ & $\mathrm{~m} \mathrm{~s}^{-1}$ \\
\hline
\end{tabular}

$h_{0}^{\mathrm{A}}=g_{0}^{\mathrm{A}}$

$h_{0}^{\mathrm{V}}=g_{0}^{\mathrm{V}}$.

Finally, evaluating Eq. (H3) at $A=1, T=T_{0}^{\mathrm{A}}, \rho=\rho_{0}^{\mathrm{A}}$ and at $A=0, T=T_{0}^{\mathrm{V}}, \rho=\rho_{0}^{\mathrm{V}}$, we get the adjustable Helmholtz energies from $g^{\mathrm{AV} \text {,id }}=f^{\mathrm{AV} \text {,id }}+P^{\mathrm{AV} \text {,id }} / \rho^{\mathrm{AV}}$ and Eqs. (H16), (H17) as

$f_{0}^{\mathrm{A}}=g_{0}^{\mathrm{A}}-P_{0} / \rho_{0}^{\mathrm{A}}=g_{0}^{\mathrm{A}}-R_{\mathrm{A}}^{\mathrm{L}} T_{0}^{\mathrm{A}}$

$f_{0}^{\mathrm{V}}=g_{0}^{\mathrm{V}}-P_{\mathrm{t}} / \rho_{0}^{\mathrm{V}}=g_{0}^{\mathrm{V}}-R_{\mathrm{W}}^{95} T_{0}^{\mathrm{V}}$.

The numerical results for these constants are listed in Table $\mathrm{H} 1$.

\section{Appendix I}

\section{Check values for the humid-air formulation}

The check values reported in Tables I1-I3 correspond to those of the draft IAPWS Guideline (IAPWS, 2010) that is the subject of an evaluation process at the time this paper is being written. The evaluation includes the reproduction of the numbers given in Tables I1-I3 by independent implementations of the equations defined in Appendix D.

\section{Appendix J}

\section{Glossary of symbols and abbreviations}

The following table summarized the formula symbols used in this paper. Additional symbols used exclusively in the appendix are explained there. 
Table I2. Numerical check values for the dry-air part, $f^{\mathrm{A}}$, and the water-vapour part, $f^{\mathrm{V}}$, of the Helmholtz function $f^{\mathrm{AV}}$ and its derivatives, Eq. (D14), for saturated air, $A=A^{\text {sat }}$, with respect to ice at $200 \mathrm{~K}$, and with respect to liquid water at 300 and $400 \mathrm{~K}$, corresponding to Table I1. The numerical functions evaluated here at given points $\left(A, T, \rho^{\mathrm{AV}}\right)$ are defined in Eqs. (B1) and (C1). The humid-air density is $\rho=\rho^{\mathrm{AV}}=\rho^{\mathrm{A}}+\rho^{\mathrm{V}}=m^{\mathrm{AV}} / V=\left(m^{\mathrm{A}}+m^{\mathrm{V}}\right) / V$.

\begin{tabular}{lllll}
\hline Quantity & Value & Value & Value & Unit \\
\hline$A$ & +0.892224944 & +0.977600624 & +0.825531379 & $\mathrm{~kg} \mathrm{~kg}^{-1}$ \\
$T$ & +200 & +300 & +400 & $\mathrm{~K}$ \\
$\rho=\rho^{\mathrm{AV}}$ & $+0.163445112 \times 10^{-4}$ & $+0.114587678 \times 10$ & $+0.793198757 \times 10$ & $\mathrm{~kg} \mathrm{~m}^{-3}$ \\
$\rho^{\mathrm{A}}=A \rho^{\mathrm{AV}}$ & $+0.145829806 \times 10^{-4}$ & $+0.112020986 \times 10$ & $+0.654810464 \times 10$ & $\mathrm{~kg} \mathrm{~m}^{-3}$ \\
$f^{\mathrm{A}}$ & $-0.740216453 \times 10^{6}$ & $-0.916320468 \times 10^{5}$ & $+0.895773442 \times 10^{5}$ & $\mathrm{~J} \mathrm{~kg}^{-1}$ \\
$\left(\partial f^{\mathrm{A}} / \partial T\right)_{\rho^{\mathrm{A}}}$ & $-0.304846375 \times 10^{4}$ & $-0.108501917 \times 10^{3}$ & $+0.193317180 \times 10^{3}$ & $\mathrm{~J} \mathrm{~kg}^{-1} \mathrm{~K}^{-1}$ \\
$\left(\partial f^{A} / \partial \rho^{\mathrm{A}}\right)_{T}$ & $+0.393770146 \times 10^{10}$ & $+0.768690856 \times 10^{5}$ & $+0.175643300 \times 10^{5}$ & $\mathrm{~J} \mathrm{~m}^{3} \mathrm{~kg}^{-2}$ \\
$\left(\partial^{2} f^{\mathrm{A}} / \partial T^{2}\right)_{\rho^{\mathrm{A}}}$ & $-0.357762609 \times 10$ & $-0.239376632 \times 10$ & $-0.181852945 \times 10$ & $\mathrm{~J} \mathrm{~kg}^{-1} \mathrm{~K}^{-2}$ \\
$\partial^{2} f^{\mathrm{A}} / \partial T \partial \rho^{\mathrm{A}}$ & $+0.196885083 \times 10^{8}$ & $+0.256804931 \times 10^{3}$ & $+0.442979471 \times 10^{2}$ & $\mathrm{~J} \mathrm{~m}^{3} \mathrm{~kg}^{-2} \mathrm{~K}^{-1}$ \\
{$\left[\partial^{2} f^{\mathrm{A}} / \partial\left(\rho^{\mathrm{A}}\right)^{2}\right]_{T}$} & $-0.270020351 \times 10^{15}$ & $-0.686404948 \times 10^{5}$ & $-0.267826171 \times 10^{4}$ & $\mathrm{~J} \mathrm{~m}^{6} \mathrm{~kg}^{-3}$ \\
$\rho^{\mathrm{V}}=(1-A) \rho^{\mathrm{AV}}$ & $+0.176153061 \times 10^{-5}$ & $+0.256669248 \times 10^{-1}$ & $+0.138388293 \times 10$ & $\mathrm{~kg} \mathrm{~m}^{-3}$ \\
$f^{\mathrm{V}}$ & $-0.202254350 \times 10^{6}$ & $-0.143157503 \times 10^{6}$ & $-0.285137783 \times 10^{6}$ & $\mathrm{~J} \mathrm{~kg}^{-1}$ \\
$\left(\partial f^{\mathrm{V}} / \partial T\right)_{\rho} \mathrm{V}$ & $-0.123787544 \times 10^{5}$ & $-0.851598239 \times 10^{4}$ & $-0.705288118 \times 10^{4}$ & $\mathrm{~J} \mathrm{~kg}^{-1} \mathrm{~K}^{-1}$ \\
$\left(\partial f^{\mathrm{V}} / \partial \rho^{\mathrm{V}}\right)_{T}$ & $+0.523995669 \times 10^{11}$ & $+0.538480918 \times 10^{7}$ & $+0.129645224 \times 10^{6}$ & $\mathrm{~J} \mathrm{~m}^{3} \mathrm{~kg}^{-2}$ \\
$\left(\partial^{2} f^{\mathrm{V}} / \partial T^{2}\right)_{\rho^{\mathrm{V}}}$ & $-0.694877601 \times 10$ & $-0.480816998 \times 10$ & $-0.411710547 \times 10$ & $\mathrm{~J} \mathrm{~kg}^{-1} \mathrm{~K}^{-2}$ \\
$\partial^{2} f^{\mathrm{V}} / \partial T \partial \rho^{\mathrm{V}}$ & $+0.262001883 \times 10^{9}$ & $+0.181489601 \times 10^{5}$ & $+0.361784532 \times 10^{3}$ & $\mathrm{~J} \mathrm{~m}^{3} \mathrm{~kg}^{-2} \mathrm{~K}^{-1}$ \\
{$\left[\partial^{2} f^{\mathrm{V}} / \partial\left(\rho^{\mathrm{V}}\right)^{2}\right]_{T}$} & $-0.297466666 \times 10^{17}$ & $-0.210185225 \times 10^{9}$ & $-0.965542132 \times 10^{5}$ & $\mathrm{~J} \mathrm{~m}^{6} \mathrm{~kg}^{-3}$ \\
\hline
\end{tabular}

Table I3. Numerical check values for the cross-virial part, $f^{\mathrm{mix}}$, and the cross-virial coefficients, $B_{\mathrm{AW}}, C_{\mathrm{AAW}}, C_{\mathrm{AWW}}$, of the Helmholtz function $f^{\mathrm{AV}}$ and its derivatives, Eq. (D14), for saturated air, $A=A^{\text {sat }}$, with respect to ice at $200 \mathrm{~K}$, and with respect to liquid water at 300 and $400 \mathrm{~K}$, corresponding to Table I1. The numerical functions evaluated here at given points $\left(A, T, \rho^{\mathrm{AV}}\right)$ are defined in Eqs. (D15-D18). The humid-air density is $\rho=\rho^{\mathrm{AV}}=\rho^{\mathrm{A}}+\rho^{\mathrm{V}}=m^{\mathrm{AV}} / V=\left(m^{\mathrm{A}}+m^{\mathrm{V}}\right) / V$.

\begin{tabular}{|c|c|c|c|c|}
\hline Quantity & Value & Value & Value & Unit \\
\hline$A$ & +0.892224944 & +0.977600624 & +0.825531379 & $\mathrm{~kg} \mathrm{~kg}^{-1}$ \\
\hline$T$ & +200 & +300 & +400 & $\mathrm{~K}$ \\
\hline$\rho=\rho^{\mathrm{AV}}$ & $+0.163445112 \times 10^{-4}$ & $+0.114587678 \times 10$ & $+0.793198757 \times 10$ & $\mathrm{~kg} \mathrm{~m}^{-3}$ \\
\hline$f^{\text {mix }}$ & $-0.786211837 \times 10^{-3}$ & $-0.711673566 \times 10$ & $-0.161985034 \times 10^{3}$ & $\mathrm{~J} \mathrm{~kg}^{-1}$ \\
\hline$\left(\partial f^{\operatorname{mix}} / \partial A\right)_{T, \rho}$ & $+0.641377589 \times 10^{-2}$ & $+0.311768501 \times 10^{3}$ & $+0.830802876 \times 10^{3}$ & $\mathrm{~J} \mathrm{~kg}^{-1}$ \\
\hline$\left(\partial f^{\mathrm{mix}} / \partial T\right)_{A, \rho}$ & $+0.456427011 \times 10^{-5}$ & $+0.441245367 \times 10^{-1}$ & $+0.178961265 \times 10$ & $\mathrm{~J} \mathrm{~kg}^{-1} \mathrm{~K}^{-1}$ \\
\hline$\left(\partial f^{\operatorname{mix}} / \partial \rho\right)_{A, T}$ & $-0.481026562 \times 10^{2}$ & $-0.623171268 \times 10$ & $-0.223365432 \times 10^{2}$ & $\mathrm{~J} \mathrm{~m}^{3} \mathrm{~kg}^{-2}$ \\
\hline$\left(\partial^{2} f^{\mathrm{mix}} / \partial A^{2}\right)_{T \rho}$ & $+0.163518397 \times 10^{-1}$ & $+0.534139178 \times 10^{3}$ & $+0.135815610 \times 10^{4}$ & $\mathrm{~J} \mathrm{~kg}^{-1}$ \\
\hline$\left(\partial^{2} f^{\mathrm{mix}} / \partial A \partial T\right)_{\rho}$ & $-0.372355251 \times 10^{-4}$ & $-0.195026098 \times 10$ & $-0.916586082 \times 10$ & $\mathrm{~J} \mathrm{~kg}^{-1} \mathrm{~K}^{-1}$ \\
\hline$\left(\partial^{2} f^{\operatorname{mix}} / \partial A \partial \rho\right)_{T}$ & $+0.392414346 \times 10^{3}$ & $+0.274152649 \times 10^{3}$ & $+0.125823778 \times 10^{3}$ & $\mathrm{~J} \mathrm{~m}^{3} \mathrm{~kg}^{-2}$ \\
\hline$\left(\partial^{2} f^{\operatorname{mix}} / \partial T^{2}\right)_{A, \rho}$ & $-0.378866039 \times 10^{-7}$ & $-0.148782305 \times 10^{-3}$ & $-0.536718536 \times 10^{-2}$ & $\mathrm{~J} \mathrm{~kg}^{-1} \mathrm{~K}^{-2}$ \\
\hline$\left(\partial^{2} f^{\operatorname{mix}} / \partial T \partial \rho\right)_{A}$ & +0.279261664 & $+0.390100462 \times 10^{-1}$ & +0.249618216 & $\mathrm{~J} \mathrm{~m}^{3} \mathrm{~kg}^{-2} \mathrm{~K}^{-1}$ \\
\hline$\left(\partial^{2} f^{\text {mix }} / \partial \rho^{2}\right)_{A, T}$ & $-0.192118914 \times 10^{2}$ & $-0.366162709 \times 10^{-1}$ & -0.482803925 & $\mathrm{~J} \mathrm{~m}^{6} \mathrm{~kg}^{-3}$ \\
\hline$B_{\mathrm{AW}}$ & $-0.784874278 \times 10^{-4}$ & $-0.295672747 \times 10^{-4}$ & $-0.100804610 \times 10^{-4}$ & $\mathrm{~m}^{3} \mathrm{~mol}^{-1}$ \\
\hline $\mathrm{d} B_{\mathrm{AW}} / \mathrm{d} T$ & $+0.848076624 \times 10^{-6}$ & $+0.280097360 \times 10^{-6}$ & $+0.135021228 \times 10^{-6}$ & $\mathrm{~m}^{3} \mathrm{~mol}^{-1} \mathrm{~K}^{-1}$ \\
\hline $\mathrm{d}^{2} B_{\mathrm{AW}} / \mathrm{d} T^{2}$ & $-0.122622146 \times 10^{-7}$ & $-0.242599241 \times 10^{-8}$ & $-0.839901729 \times 10^{-9}$ & $\mathrm{~m}^{3} \mathrm{~mol}^{-1} \mathrm{~K}^{-2}$ \\
\hline$C_{\mathrm{AAW}}$ & $+0.105493575 \times 10^{-8}$ & $+0.801977741 \times 10^{-9}$ & $+0.672018172 \times 10^{-9}$ & $\mathrm{~m}^{6} \mathrm{~mol}^{-2}$ \\
\hline $\mathrm{d} C_{\mathrm{AAW}}$ & $-0.152535000 \times 10^{-11}$ & $-0.196103457 \times 10^{-11}$ & $-0.812416406 \times 10^{-12}$ & $\mathrm{~m}^{6} \mathrm{~mol}^{-2} \mathrm{~K}^{-1}$ \\
\hline $\mathrm{d}^{2} C_{\mathrm{AAW}} / \mathrm{d} T^{2}$ & $-0.113436375 \times 10^{-12}$ & $+0.170055638 \times 10^{-13}$ & $+0.683147461 \times 10^{-14}$ & $\mathrm{~m}^{6} \mathrm{~mol}^{-2} \mathrm{~K}^{-2}$ \\
\hline$C_{\mathrm{AWW}}$ & $-0.349872634 \times 10^{-5}$ & $-0.115552784 \times 10^{-6}$ & $-0.200806021 \times 10^{-7}$ & $\mathrm{~m}^{6} \mathrm{~mol}^{-2}$ \\
\hline $\mathrm{d} C_{\mathrm{AWv}}$ & $+0.188025052 \times 10^{-6}$ & $+0.261363278 \times 10^{-8}$ & $+0.274535403 \times 10^{-9}$ & $\mathrm{~m}^{6} \mathrm{~mol}^{-2} \mathrm{~K}^{-1}$ \\
\hline $\mathrm{d}^{2} C_{\mathrm{AWW}} / \mathrm{d} T^{2}$ & $-0.124996856 \times 10^{-7}$ & $-0.751334582 \times 10^{-10}$ & $-0.491763910 \times 10^{-11}$ & $\mathrm{~m}^{6} \mathrm{~mol}^{-2} \mathrm{~K}^{-2}$ \\
\hline
\end{tabular}


Table J1. Formula symbols used in the paper.

\begin{tabular}{|c|c|c|}
\hline Symbol & Quantity & Unit \\
\hline$A$ & mass fraction of dry air in humid air & $\mathrm{kg} \mathrm{kg}^{-1}$ \\
\hline$A^{\mathrm{sat}}$ & mass fraction of dry air in saturated humid air & $\mathrm{kg} \mathrm{kg}^{-1}$ \\
\hline$A_{\mathrm{AW}}^{\mathrm{sat}}$ & mass fraction of dry air in the humid-air part of wet air & $\mathrm{kg} \mathrm{kg}^{-1}$ \\
\hline$A_{\mathrm{AI}}^{\mathrm{Aw}}$ & mass fraction of dry air in the humid-air part of ice air & $\mathrm{kg} \mathrm{kg}^{-1}$ \\
\hline$A_{\mathrm{SO}}$ & standard-ocean air fraction, $A_{\mathrm{SO}}=0.996293104 \mathrm{~kg} \mathrm{~kg}^{-1}$ & $\mathrm{~kg} \mathrm{~kg}^{-1}$ \\
\hline$a_{\mathrm{W}}$ & activity of water & \\
\hline$B_{\mathrm{AW}}$ & molar second virial coefficient air-water & $\mathrm{m}^{3} \mathrm{~mol}^{-1}$ \\
\hline$C_{\mathrm{AAW}}$ & molar third virial coefficient air-air-water & $\mathrm{m}^{6} \mathrm{~mol}^{-2}$ \\
\hline$C_{\mathrm{AWW}}$ & molar third virial coefficient air-water-water & $\mathrm{m}^{6} \mathrm{~mol}^{-2}$ \\
\hline$c$ & sound speed & $\mathrm{m} \mathrm{s}^{-1}$ \\
\hline$c_{P}^{\mathrm{Ar}, \mathrm{id}}$ & specific isobaric heat capacity of Argon & $\mathrm{J} \mathrm{kg}^{-1} \mathrm{~K}^{-1}$ \\
\hline$c_{P}^{\mathrm{AV}}$ & specific isobaric heat capacity of humd air & $\mathrm{J} \mathrm{kg}^{-1} \mathrm{~K}^{-1}$ \\
\hline$c_{P}^{\mathrm{AW}}$ & specific isobaric heat capacity of wet air & $\mathrm{J} \mathrm{kg}^{-1} \mathrm{~K}^{-1}$ \\
\hline$c_{P}^{\mathrm{SA}}$ & specific isobaric heat capacity of sea air & $\mathrm{J} \mathrm{kg}^{-1} \mathrm{~K}^{-1}$ \\
\hline$c_{P}^{\mathrm{SIA}}$ & specific isobaric heat capacity of sea-ice air & $\mathrm{J} \mathrm{kg}^{-1} \mathrm{~K}^{-1}$ \\
\hline$D_{\mathrm{A}}$ & chemical air coefficient of humid air, Eq. (6.8) & $\mathrm{J} \mathrm{kg}^{-1}$ \\
\hline$D_{\mathrm{AS}}$ & chemical air-salt coefficient of sea air, Eq. (6.12) & $\mathrm{J} \mathrm{kg}^{-1}$ \\
\hline$D_{\mathrm{S}}$ & chemical sea-salt coefficient of seawater, Eq. (6.9) & $\mathrm{J} \mathrm{kg}^{-1}$ \\
\hline$F^{\mathrm{A}}$ & Helmholtz energy of dry air & $\mathrm{J}$ \\
\hline$F^{\mathrm{AV}}$ & Helmholtz energy of humid air & $\mathrm{J}$ \\
\hline$f$ & specific Helmholtz energy & $\mathrm{J} \mathrm{kg}^{-1}$ \\
\hline$f^{\mathrm{A}}$ & specific Helmholtz energy of dry air & $\mathrm{J} \mathrm{kg}^{-1}$ \\
\hline$f^{\mathrm{A}, \mathrm{mol}}$ & molar Helmholtz energy of dry air & $\mathrm{J} \mathrm{mol}^{-1}$ \\
\hline$f^{\mathrm{AV}}$ & specific Helmholtz energy of humid air & $\mathrm{J} \mathrm{kg}^{-1}$ \\
\hline$f_{\mathrm{AV}}$ & fugacity of humid air & $\mathrm{Pa}$ \\
\hline$f^{\mathrm{F}}$ & specific Helmholtz energy of fluid water & $\mathrm{J} \mathrm{kg}^{-1}$ \\
\hline$f^{\mathrm{V}}$ & specific Helmholtz energy of water vapour & $\mathrm{J} \mathrm{kg}^{-1}$ \\
\hline$f_{\mathrm{V}}$ & fugacity of vapour in humid air & $\mathrm{Pa}$ \\
\hline$f_{\mathrm{V}}^{0}$ & reference fugacity of vapour in humid air & $\mathrm{Pa}$ \\
\hline$f_{\mathrm{V}}^{\mathrm{SA}}$ & fugacity of vapour in sea air & $\mathrm{Pa}$ \\
\hline$f_{\mathrm{V}}^{\text {sat }}$ & saturation fugacity of vapour in humid air & $\mathrm{Pa}$ \\
\hline$g$ & specific Gibbs energy & $\mathrm{J} \mathrm{kg}^{-1}$ \\
\hline$g^{\mathrm{AV}}$ & specific Gibbs energy of humid air & $\mathrm{J} \mathrm{kg}^{-1}$ \\
\hline$g^{A V, i d}$ & specific Gibbs energy of ideal-gas humid air & $\mathrm{J} \mathrm{kg}^{-1}$ \\
\hline$g$ Ih & specific Gibbs energy of ice Ih & $\mathrm{J} \mathrm{kg}^{-1}$ \\
\hline$g^{S}$ & saline part of the specific Gibbs energy of seawater & $\mathrm{J} \mathrm{kg}^{-1}$ \\
\hline$g^{\circ} \mathrm{SA}$ & specific Gibbs energy of sea air & $\mathrm{J} \mathrm{kg}^{-1}$ \\
\hline$h$ & specific enthalpy & $\mathrm{J} \mathrm{kg}^{-1}$ \\
\hline$h^{\mathrm{AV}}$ & specific enthalpy of humid air & $\mathrm{J} \mathrm{kg}^{-1}$ \\
\hline$H_{\mathrm{V}}^{\mathrm{AV}}$ & partial specific enthalpy of water vapour in humid air & $\mathrm{J} \mathrm{kg}^{-1}$ \\
\hline$h^{\mathrm{AW}}$ & specific enthalpy of wet air & $\mathrm{J} \mathrm{kg}^{-1}$ \\
\hline$h^{\mathrm{Ih}}$ & specific enthalpy of ice Ih & $\mathrm{J} \mathrm{kg}^{-1}$ \\
\hline$h^{\mathrm{SA}}$ & specific enthalpy of sea air & $\mathrm{J} \mathrm{kg}^{-1}$ \\
\hline$h^{\mathrm{SIA}}$ & specific enthalpy of sea-ice air & $\mathrm{J} \mathrm{kg}^{-1}$ \\
\hline$h^{\mathrm{SW}}$ & specific enthalpy of seawater & $\mathrm{J} \mathrm{kg}^{-1}$ \\
\hline$H_{\mathrm{W}}^{\mathrm{SW}}$ & partial specific enthalpy of water in seawater & $\mathrm{J} \mathrm{kg}^{-1}$ \\
\hline$L_{P}^{\mathrm{Al}}$ & isobaric specific latent heat of ice air & $\mathrm{J} \mathrm{kg}^{-1}$ \\
\hline$L_{P}^{\mathrm{ASS}}$ & isobaric specific latent heat of sea air & $\mathrm{J} \mathrm{kg}^{-1}$ \\
\hline$L_{P}^{\mathrm{AW}}$ & isobaric specific latent heat of wet air & $\mathrm{J} \mathrm{kg}^{-1}$ \\
\hline$L_{P}^{\mathrm{SI}}$ & isobaric specific latent heat of sea ice & $\mathrm{J} \mathrm{kg}^{-1}$ \\
\hline$L_{P}^{S I A}$ & isobaric specific latent heat of sea-ice air & $\mathrm{J} \mathrm{kg}^{-1}$ \\
\hline$M^{\mathrm{A}}$ & molar mass of dry air, $M^{\mathrm{A}}=0.02896546 \mathrm{~kg} \mathrm{~mol}^{-1}$ & $\mathrm{~kg} \mathrm{~mol}^{-1}$ \\
\hline$M^{\mathrm{A}, \mathrm{L}}$ & $\begin{array}{l}\text { molar mass of dry air used in Lemmon et al. (2000), } \\
M^{\mathrm{A}, \mathrm{L}}=0.0289586 \mathrm{~kg} \mathrm{~mol}^{-1}\end{array}$ & $\mathrm{~kg} \mathrm{~mol}^{-1}$ \\
\hline$M^{\mathrm{AV}}$ & molar mass of humid air & $\mathrm{kg} \mathrm{mol}^{-1}$ \\
\hline$M^{S}$ & molar mass of sea salt, $M^{\mathrm{S}}=0.031404 \mathrm{~kg} \mathrm{~mol}^{-1}$ & $\mathrm{~kg} \mathrm{~mol}^{-1}$ \\
\hline
\end{tabular}


Table J1. Continued.

\begin{tabular}{|c|c|c|}
\hline Symbol & Quantity & Unit \\
\hline$M^{\mathrm{W}}$ & molar mass of water, $M^{\mathrm{W}}=0.018015268 \mathrm{~kg} \mathrm{~mol}^{-1}$ & $\mathrm{~kg} \mathrm{~mol}^{-1}$ \\
\hline$m^{\mathrm{A}}$ & mass of dry air & $\mathrm{kg}$ \\
\hline$m^{\mathrm{Ih}}$ & mass of ice Ih & $\mathrm{kg}$ \\
\hline$m^{\mathrm{AV}}$ & mass of humid air & $\mathrm{kg}$ \\
\hline$m^{\mathrm{S}}$ & mass of sea salt & $\mathrm{kg}$ \\
\hline$m^{\mathrm{SA}}$ & mass of sea air & $\mathrm{kg}$ \\
\hline$m^{\text {SIA }}$ & mass of sea-ice air & $\mathrm{kg}$ \\
\hline$m^{\mathrm{SW}}$ & mass of seawater & $\mathrm{kg}$ \\
\hline$m_{\mathrm{SW}}$ & molality of seawater & $\mathrm{mol} \mathrm{kg}^{-1}$ \\
\hline$m^{\mathrm{W}}$ & mass of liquid water & $\mathrm{kg}$ \\
\hline$m^{\mathrm{V}}$ & mass of water vapour & $\mathrm{kg}$ \\
\hline$P$ & absolute pressure & $\mathrm{Pa}$ \\
\hline$P^{\mathrm{a}}$ & partial pressure of the component "a" & $\mathrm{Pa}$ \\
\hline$P_{\mathrm{DP}}$ & dewpoint pressure & $\mathrm{Pa}$ \\
\hline$P_{\mathrm{f}}$ & freezing point pressure & $\mathrm{Pa}$ \\
\hline$P_{0}, P_{\mathrm{SO}}$ & standard ocean surface pressure, $P_{0}=101325 \mathrm{~Pa}$ & $\mathrm{~Pa}$ \\
\hline$P^{\mathrm{V}}$ & partial pressure of vapour & $\mathrm{Pa}$ \\
\hline$q$ & specific humidity & $\mathrm{kg} \mathrm{kg}^{-1}$ \\
\hline$R$ & molar gas constant, $R=8.314472 \mathrm{~J} \mathrm{~mol}^{-1} \mathrm{~K}^{-1}$ & $\mathrm{~J} \mathrm{~mol}^{-1} \mathrm{~K}^{-1}$ \\
\hline$R^{\mathrm{L}}$ & $\begin{array}{l}\text { molar gas constant used in Lemmon et al. (2000), } \\
R^{\mathrm{L}}=8.31451 \mathrm{~J} \mathrm{~mol}^{-1} \mathrm{~K}^{-1}\end{array}$ & $\mathrm{~J} \mathrm{~mol}^{-1} \mathrm{~K}^{-1}$ \\
\hline$R_{\mathrm{A}}$ & specific gas constant of dry air, $R_{\mathrm{A}}=287.0478 \mathrm{~J} \mathrm{~kg}^{-1} \mathrm{~K}^{-1}$ & $\mathrm{~J} \mathrm{~mol}^{-1} \mathrm{~K}^{-1}$ \\
\hline$R_{\mathrm{A}}^{\mathrm{L}}$ & $\begin{array}{l}\text { specific gas constant of dry air used in Lemmon et al. } \\
(2000), R_{\mathrm{A}}^{\mathrm{L}}=R^{\mathrm{L}} / M^{\mathrm{A}, \mathrm{L}}\end{array}$ & $\mathrm{J} \mathrm{mol}^{-1} \mathrm{~K}^{-1}$ \\
\hline$R_{\mathrm{AV}}$ & specific gas "constant" of humid air & $\mathrm{J} \mathrm{mol}^{-1} \mathrm{~K}^{-1}$ \\
\hline RH & relative humidity & \\
\hline$R H_{\mathrm{CCT}}$ & relative humidity, CCT definition & \\
\hline$R H_{\mathrm{WMO}}$ & relative humidity, WMO definition & \\
\hline$R_{\mathrm{W}}$ & specific gas constant of water, $R_{\mathrm{W}}=461.52364 \mathrm{~J} \mathrm{~kg}^{-1} \mathrm{~K}^{-1}$ & $\mathrm{~J} \mathrm{~mol}^{-1} \mathrm{~K}^{-1}$ \\
\hline$R_{\mathrm{W}}^{95}$ & $\begin{array}{l}\text { specific gas constant of water used in IAPWS-95, } \\
R_{\mathrm{W}}^{95}=461.51805 \mathrm{~J} \mathrm{~kg}^{-1} \mathrm{~K}^{-1}\end{array}$ & $\mathrm{~J} \mathrm{~mol}^{-1} \mathrm{~K}^{-1}$ \\
\hline$r$ & humidity ratio, mixing ratio & $\mathrm{kg} \mathrm{kg}^{-1}$ \\
\hline$S_{\mathrm{A}}$ & absolute salinity: mass fraction of sea salt in seawater & $\mathrm{kg} \mathrm{kg}^{-1}$ \\
\hline$S_{\mathrm{P}}$ & practical salinity & \\
\hline$S_{\mathrm{SO}}$ & $\begin{array}{l}\text { standard-ocean absolute salinity, } \\
S_{\mathrm{SO}}=0.03516504 \mathrm{~kg} \mathrm{~kg}^{-1}\end{array}$ & $\mathrm{~kg} \mathrm{~kg}^{-1}$ \\
\hline$s$ & specific entropy & $\mathrm{J} \mathrm{kg}^{-1} \mathrm{~K}^{-1}$ \\
\hline$s^{\mathrm{AV}}$ & specific entropy of humid air & $\mathrm{J} \mathrm{kg}^{-1} \mathrm{~K}^{-1}$ \\
\hline$s^{\mathrm{AW}}$ & specific entropy of wet air & $\mathrm{J} \mathrm{kg}^{-1} \mathrm{~K}^{-1}$ \\
\hline$s^{\text {SIA }}$ & specific entropy of sea-ice air & $\mathrm{J} \mathrm{kg}^{-1} \mathrm{~K}^{-1}$ \\
\hline$s^{\mathrm{SA}}$ & specific entropy of sea air & $\mathrm{J} \mathrm{kg}^{-1} \mathrm{~K}^{-1}$ \\
\hline$s^{\mathrm{SW}}$ & specific entropy of seawater & $\mathrm{J} \mathrm{kg}^{-1} \mathrm{~K}^{-1}$ \\
\hline$T$ & absolute temperature & $\mathrm{K}$ \\
\hline$T_{\mathrm{DP}}$ & dewpoint temperature & $\mathrm{K}$ \\
\hline$T_{\mathrm{f}}$ & freezing point temperature & $\mathrm{K}$ \\
\hline$T_{0}, T_{\mathrm{SO}}$ & standard ocean temperature, $T_{0}=273.15 \mathrm{~K}$ & $\mathrm{~K}$ \\
\hline$T_{\mathrm{V}}$ & virtual temperature & $\mathrm{K}$ \\
\hline$V$ & volume & $\mathrm{m}^{3}$ \\
\hline$v$ & specific volume & $\mathrm{m}^{3} \mathrm{~kg}^{-1}$ \\
\hline$v^{\mathrm{AV}}$ & specific volume of humid air & $\mathrm{m}^{3} \mathrm{~kg}^{-1}$ \\
\hline$v^{\mathrm{AW}}$ & specific volume of wet air & $\mathrm{m}^{3} \mathrm{~kg}^{-1}$ \\
\hline$v^{\mathrm{SA}}$ & specific volume of sea air & $\mathrm{m}^{3} \mathrm{~kg}^{-1}$ \\
\hline$v^{\mathrm{SIA}}$ & specific volume of sea-ice air & $\mathrm{m}^{3} \mathrm{~kg}^{-1}$ \\
\hline$v^{\mathrm{SW}}$ & specific volume of seawater & $\mathrm{m}^{3} \mathrm{~kg}^{-1}$ \\
\hline
\end{tabular}


Table J1. Continued.

\begin{tabular}{|c|c|c|}
\hline Symbol & Quantity & Unit \\
\hline$w^{\mathrm{A}}$ & mass fraction of air in sea/wet air & $\mathrm{kg} \mathrm{kg}^{-1}$ \\
\hline$w^{\mathrm{AV}}$ & mass fraction of humid air in sea/wet air & $\mathrm{kg} \mathrm{kg}^{-1}$ \\
\hline$w^{\text {Ih }}$ & mass fraction of ice in ice/sea-ice air & $\mathrm{kg} \mathrm{kg}^{-1}$ \\
\hline$w^{\mathrm{S}}$ & mass fraction of sea salt in sea air & $\mathrm{kg} \mathrm{kg}^{-1}$ \\
\hline$w^{\mathrm{SW}}$ & mass fraction of seawater in sea air & $\mathrm{kg} \mathrm{kg}^{-1}$ \\
\hline$w^{\mathrm{W}}$ & mass fraction of liquid water in wet air & $\mathrm{kg} \mathrm{kg}^{-1}$ \\
\hline$x_{a}$ & mole fraction of a component "a" & $\mathrm{mol} \mathrm{mol}^{-1}$ \\
\hline$x_{\mathrm{V}}^{\mathrm{AV}}$ & mole fraction of vapour in humid air & $\mathrm{mol} \mathrm{mol}^{-1}$ \\
\hline$x_{\mathrm{V}}^{\mathrm{AV}, \mathrm{sat}}$ & saturation mole fraction of vapour in humid air & $\mathrm{mol} \mathrm{mol}^{-1}$ \\
\hline$Z_{\mathrm{AV}}$ & compressibility factor of humid air & \\
\hline$z$ & any thermodynamic property & \\
\hline$\alpha^{\mathrm{AW}}$ & thermal expansion coefficient of wet air & $\mathrm{K}^{-1}$ \\
\hline$\delta_{P}^{\mathrm{AI}}$ & isobaric drying rate of ice air & $\mathrm{K}^{-1}$ \\
\hline$\delta_{T}^{\mathrm{AI}}$ & isothermal drying rate of ice air & $\mathrm{Pa}^{-1}$ \\
\hline$\delta_{P}^{\mathrm{AWW}}$ & isobaric drying rate of wet air & $\mathrm{K}^{-1}$ \\
\hline$\delta_{s}^{\mathrm{AW}}$ & adiabatic drying rate of wet air & $\mathrm{Pa}^{-1}$ \\
\hline$\delta_{T}^{\mathrm{AW}}$ & isothermal drying rate of wet air & $\mathrm{Pa}^{-1}$ \\
\hline$\varepsilon_{P}^{\text {SIA }}$ & isobaric melting rate & $\mathrm{K}^{-1}$ \\
\hline$\varepsilon_{T}^{\mathrm{SIA}}$ & isothermal melting rate & $\mathrm{Pa}^{-1}$ \\
\hline$\varphi$ & relative fugacity & \\
\hline$\varphi^{\mathrm{SA}}$ & relative fugacity of sea air & \\
\hline$\phi^{\mathrm{SW}}$ & osmotic coefficient of seawater & \\
\hline$\Gamma$ & adiabatic lapse rate & $\mathrm{K} \mathrm{Pa}^{-1}$ \\
\hline$\Gamma^{\mathrm{AV}}$ & adiabatic lapse rate of humid air & $\mathrm{K} \mathrm{Pa}^{-1}$ \\
\hline$\Gamma_{\mathrm{V}}^{\mathrm{AV}}$ & virtual adiabatic lapse rate of humid air & $\mathrm{K} \mathrm{Pa}^{-1}$ \\
\hline$\Gamma^{\mathrm{SA}}$ & adiabatic lapse rate of sea air & $\mathrm{K} \mathrm{Pa}^{-1}$ \\
\hline$\kappa_{s}^{\mathrm{AV}}$ & isentropic compressibility of humid air & $\mathrm{Pa}^{-1}$ \\
\hline$\kappa_{T}^{\mathrm{AV}}$ & isothermal compressibility of humid air & $\mathrm{Pa}^{-1}$ \\
\hline$\kappa_{T}^{\mathrm{AW}}$ & isothermal compressibility of wet air & $\mathrm{Pa}^{-1}$ \\
\hline$\kappa_{T}^{\mathrm{SA}}$ & isothermal compressibility of sea air & $\mathrm{Pa}^{-1}$ \\
\hline$\kappa_{T}^{\mathrm{SW}}$ & isothermal compressibility of seawater & $\mathrm{Pa}^{-1}$ \\
\hline$\Lambda_{\mathrm{AI}}$ & ice-air phase-transition latency operator & \\
\hline$\Lambda_{\mathrm{AS}}$ & air-sea phase-transition latency operator & \\
\hline$\Lambda_{\mathrm{AW}}$ & wet-air phase-transition latency operator & \\
\hline$\Lambda_{\mathrm{SI}}$ & sea-ice phase-transition latency operator & \\
\hline$\Lambda_{\mathrm{WI}}$ & water-ice phase-transition latency operator & \\
\hline$\lambda_{\mathrm{V}}$ & absolute activity of vapour & \\
\hline$\mu^{\mathrm{A}}$ & chemical potential of air in humid air & $\mathrm{J} \mathrm{kg}^{-1}$ \\
\hline$\mu^{\mathrm{Ih}}$ & chemical potential of ice Ih & $\mathrm{J} \mathrm{kg}^{-1}$ \\
\hline$\mu^{\mathrm{V}}$ & chemical potential of vapour in humid air & $\mathrm{J} \mathrm{kg}^{-1}$ \\
\hline$\mu^{\mathrm{V}, \mathrm{id}}$ & chemical potential of ideal-gas vapour & $\mathrm{J} \mathrm{kg}^{-1}$ \\
\hline$\mu^{\mathrm{W}}$ & chemical potential of liquid water & $\mathrm{J} \mathrm{kg}^{-1}$ \\
\hline$\rho$ & density & $\mathrm{kg} \mathrm{m}^{-3}$ \\
\hline$\rho^{\mathrm{A}}$ & density of dry air & $\mathrm{kg} \mathrm{m}^{-3}$ \\
\hline$\rho^{\mathrm{AV}}$ & density of humid air & $\mathrm{kg} \mathrm{m}^{-3}$ \\
\hline$\rho^{\mathrm{mol}}$ & molar density & $\mathrm{mol} \mathrm{m}^{-3}$ \\
\hline$\rho^{\mathrm{SA}}$ & density of sea air & $\mathrm{kg} \mathrm{m}^{-3}$ \\
\hline$\rho^{\mathrm{V}}$ & density of water vapour & $\mathrm{kg} \mathrm{m}^{-3}$ \\
\hline$\rho^{\mathrm{W}}$ & density of liquid water & $\mathrm{kg} \mathrm{m}^{-3}$ \\
\hline$\theta^{\mathrm{AV}}$ & potential temperature of humid air & $\mathrm{K}$ \\
\hline$\theta_{\mathrm{e}}^{\mathrm{AV}}$ & equivalent potential temperature of humid air & K \\
\hline$\theta^{\mathrm{AW}}$ & potential temperature of wet air & $\mathrm{K}$ \\
\hline$\chi$ & adiabatic humidification rate & $\mathrm{K}^{-1}$ \\
\hline
\end{tabular}


Table J2. Abbreviations used in the paper.

\begin{tabular}{|c|c|}
\hline Abbreviation & Comment \\
\hline AMOR-92 & Atlantic Measurement of Ocean Radiation 1992 \\
\hline $\mathrm{CCT}$ & $\begin{array}{l}\text { Consultative Committee for Thermometry } \\
\text { http://www.bipm.org/en/committees/cc/cct/ }\end{array}$ \\
\hline CIPM & $\begin{array}{l}\text { International Committee for Weights and Measures } \\
\text { http://www.bipm.org/en/committees/cipm/ }\end{array}$ \\
\hline CIPM-2007 & CIPM equation of state of moist air, Picard et al. (2008) \\
\hline EOS-80 & $\begin{array}{l}\text { International Equation of State of Seawater 1980 } \\
\text { http://unesdoc.unesco.org/images/0005/000598/059832EB.pdf }\end{array}$ \\
\hline IAPSO & $\begin{array}{l}\text { International Association for the Physical Sciences of the Ocean } \\
\text { http://iapso.sweweb.net }\end{array}$ \\
\hline IAPWS & $\begin{array}{l}\text { The International Association for the Properties of Water and Steam } \\
\text { http://www.iapws.org }\end{array}$ \\
\hline IAPWS-95 & $\begin{array}{l}\text { Revised Release on the IAPWS Formulation } 1995 \text { for the Thermodynamic } \\
\text { Properties of Ordinary Water Substance for General and Scientific Use } \\
\text { http://www.iapws.org/relguide/IAPWS95-Rev.pdf }\end{array}$ \\
\hline ICL & isentropic condensation level \\
\hline ICT & isentropic condensation temperature \\
\hline IFL & isentropic freezing level \\
\hline IML & isentropic melting level \\
\hline IOC & $\begin{array}{l}\text { Intergovernmental Oceanographic Commission } \\
\text { http://ioc-unesco.org }\end{array}$ \\
\hline IPTS-68 & $\begin{array}{l}\text { International Practical Temperature Scale of } 1968 \\
\text { Goldberg and Weir (1992) }\end{array}$ \\
\hline ITS-90 & $\begin{array}{l}\text { International Temperature Scale of } 1990 \\
\text { http://www.bipm.org/en/publications/its-90.html }\end{array}$ \\
\hline IUPAC & $\begin{array}{l}\text { International Union of Pure and Applied Chemistry } \\
\text { http://www.iupac.org }\end{array}$ \\
\hline PSS-78 & Practical Salinity Scale 1978, Unesco (1981) \\
\hline RS & radiosonde \\
\hline UTC & Universal Coordinated Time \\
\hline SCOR & $\begin{array}{l}\text { Scientific Committee on Oceanic Research } \\
\text { http://www.scor-int.org }\end{array}$ \\
\hline SLP & sea-level pressure \\
\hline SP & saturation point \\
\hline SSS & sea-surface salinity \\
\hline SST & sea-surface temperature \\
\hline TEOS-10 & $\begin{array}{l}\text { International Thermodynamic Equation of Seawater } \\
\text { http://www.teos-10.org }\end{array}$ \\
\hline VB & Visual Basic \\
\hline WG127 & $\begin{array}{l}\text { SCOR/IAPSO Working Group } 127 \text { on } \\
\text { Thermodynamics and Equation of State of Seawater } \\
\text { http://www.scor-int.org/Working_Groups/wg127.htm }\end{array}$ \\
\hline WMO & $\begin{array}{l}\text { World Meteorological Organization } \\
\text { http://www.wmo.ch }\end{array}$ \\
\hline
\end{tabular}


Acknowledgements. The authors are indebted to Allan H. Harvey and Trevor J. McDougall for essential contributions to this paper. They thank Nicholas R. Nalli for kindly providing AEROSE radiosonde data, Eric W. Lemmon for assisting with digital dry-air data, and Paul Durack and Ingrid Hartmann for hints on additional literature. Various comments on the manuscript given by Frank Debogorski as well as by the anonymous reviewers are gratefully acknowledged. This paper contributes to the tasks of the SCOR/IAPSO WG127 on Thermodynamics and Equation of State of Seawater.

The software library used and described in this paper is available in the form of Fortran or Visual Basic source code from the digital supplement of Wright et al. (2009) and as a beta version from the authors upon request.

Edited by: R. Tailleux

\section{References}

Alberty, R. A.: Use of Legendre transforms in chemical thermodynamics, Pure Appl. Chem., 73, 1349-1380, 2001.

Alexandrov, A. A. and Orlov, K. A.: The thermodynamic properties of humid air at high pressure and temperature, Thermal Eng., 54, 548-551, 2007.

Ambrose, D. and Lawrenson, I. J.: The vapour pressure of water, J. Chem. Thermodyn., 4, 755-761, 1972.

Arons, A. B. and Kientzler, C. F.: Vapor pressure of sea-salt solutions, Trans. Am. Geophys. Union, 35, 722-728, 1954.

Baosen, L.: The latent and sensible heat fluxes over the western tropical Pacific and its relationship to ENSO, Adv. Atmosph. Sci., 6, 467-474, 1989.

Battino, R., Rettich, T. R., and Tominaga, T.: The solubility of nitrogen and air in liquids, J. Phys. Chem. Ref. Data, 13, 563-600, 1984.

Bezold, W. v.: Zur Thermodynamik der Atmosphaere. Zweite Mittheilung. Potentielle Temperatur. Verticaler Temperaturgradient. Zusammengesetzte Convection, Sitz. ber. Königl. Preuss. Akad. Wiss. Berlin, 46, 1189-1206, online available at: http://bibliothek.bbaw.de/bibliothek-digital/digitalequellen/ schriften/anzeige/index_html?band=10-sitz/1888-2\\&seite: int $=530,1888$.

Blandamer, M. J., Engberts, J. B. F. N., Gleeson, P. T., and Reis, J. C. R.: Activity of water in aqueous systems; A frequently neglected property, Chem. Soc. Rev. 34, 440-458, 2005.

Bolton, D.: The computation of equivalent potential temperature, Mon. Weather Rev., 108, 1046-1053, 1980.

Calvert, J. G.: Glossary of atmospheric chemistry terms (Recommendations 1990), Pure Appl. Chem., 62, 2167-2219, 1990.

Cochrane, T. T. and Cochrane, T. A.: Sea Water Dilution and its Influence on Climatic Change, J. Climatic Change, submitted, 2009.

Cooper, J. R.: Representation of the ideal-gas thermodynamic properties of water, Int. J. Thermophys., 3, 35-43, 1982.
Curry, R., Dickson, B., and Yashayev, I.: A change in the freshwater balance of the Atlantic Ocean over the past four decades, Nature, 426, 826-829, 2003.

Dai, A.: Recent Climatology, Variability, and Trends in Global Surface Humidity, J. Climate, 19, 3589-3605, 2006.

Davis, R. S.: Equation for the determination of the density of moist air (1981/91), Metrologia, 29, 67-70, 1992.

Defant, A.: Physical Oceanography, Vol. I, Pergamon Press, Oxford, 1961.

De Groot, S. R. and Mazur, P.: Non-equilibrium thermodynamics, Dover Publications, New York, 1984.

Dietrich, G., Kalle, K., Krauss, W., and Siedler, G.: Allgemeine Meereskunde, Gebr. Bornträger, Berlin, Stuttgart, 1975.

Doherty, B. T. and Kester, D. R.: Freezing Point of Seawater, J. Mar. Res., 32, 285-300, 1974.

Dressler, A. E., Zhang, Z., and Yang, P.: Water-vapor climate feedback inferred from climate fluctuations. 2003-2008, Geophys. Res. Lett., 35, L20704, doi:10.1029/2008GL035333, 2008.

Durack, P. J. and Wijffels, S. E.: Fifty-year trends in global ocean salinities and their relationship to broadscale warming, J. Climate, submitted, 2009.

Ebeling, W. and Feistel, R.: Physik der Selbstorganisation und Evolution, Akademie-Verlag, Berlin, 1982.

Emanuel, K. A.: Atmospheric convection, University Press, New York, Oxford, 1994.

Ewing, M. B., Lilley, T. H., Olofsson, G. M., Ratzsch, M. T., and Somsen, G.: Standard quantities in chemical thermodynamics. Fugacities, activities and equilibrium constants for pure and mixed phases (IUPAC Recommendations 1994), Pure Appl. Chem., 66, 533-552, 1994.

Falkenhagen, H., Ebeling, W., and Hertz, H. G.: Theorie der Elektrolyte, S. Hirzel, Leipzig, 1971.

Feistel, R.: Equilibrium thermodynamics of seawater revisited, Progr. Oceanogr., 31, 101-179, 1993.

Feistel, R.: A Gibbs Function for Seawater Thermodynamics for -6 to $80^{\circ} \mathrm{C}$ and Salinity up to $120 \mathrm{~g} / \mathrm{kg}$, Deep-Sea Res. I, 55, 1639-1671, 2008a.

Feistel, R.: Thermodynamics of Water, Vapour, Ice and Seawater, Accred. Qual. Assur., 13, 593-599, 2008b.

Feistel, R.: Extended Equation of State for Seawater at Elevated Temperature and Salinity, Desalination, 250, 14-18, 2010.

Feistel, R. and Feistel, S.: Die Ostsee als thermodynamisches System, in: L. Schimansky-Geier, H. Malchow, T. Pöschel (Hrsg.), Irreversible Prozesse und Selbstorganisation, LogosVerlag, Berlin, 247-264, 2006.

Feistel, R. and Hagen, E.: On the GIBBS thermodynamic potential of seawater, Progr. Oceanogr., 36, 249-327, 1995.

Feistel, R. and Hagen, E.: A Gibbs thermodynamic potential of sea ice, Cold Reg. Sci. Technol., 28, 83-142, 1998.

Feistel, R. and Marion, G. M.: A Gibbs-Pitzer Function for HighSalinity Seawater Thermodynamics, Prog. Oceanogr., 74, 515539, 2007.

Feistel, R. and Wagner, W.: High-pressure thermodynamic Gibbs functions of ice and sea ice, J Mar. Res., 63, 95-139, 2005.

Feistel, R. and Wagner, W.: A new equation of state for $\mathrm{H}_{2} \mathrm{O}$ ice Ih, J. Phys. Chem. Ref. Data, 35, 1021-1047, 2006.

Feistel, R. and Wagner, W.: Sublimation pressure and sublimation enthalpy of $\mathrm{H}_{2} \mathrm{O}$ ice Ih between 0 and $273.16 \mathrm{~K}$, Geochim. Cosmochim. Acta, 71, 36-45, 2007. 
Feistel, R., Wright, D. G., Jackett, D. R., Miyagawa, K., Reissmann, J. H., Wagner, W., Overhoff, U., Guder, C., Tchijov, V., Feistel, A., and Marion, G. M.: Numerical implementation and oceanographic application of the thermodynamic potentials of water, vapour, ice, seawater and air. Part I: Background and Equations, Ocean Sci., submitted, 2009.

Feistel, R., Feistel, S., Nausch, G., Szaron, J., Lysiak-Pastuszak, E., and Ærtebjerg, G.: BALTIC: Monthly time series 1900-2005, in: State and Evolution of the Baltic Sea, 1952 - 2005. A Detailed 50-Year Survey of Meteorology and Climate, Physics, Chemistry, Biology, and Marine Environment, edited by: Feistel, R., Nausch, G., and Wasmund, N., John Wiley \& Sons, Inc., Hoboken, 311-336, 2008a.

Feistel, R., Wright, D. G., Miyagawa, K., Harvey, A. H., Hruby, J., Jackett, D. R., McDougall, T. J., and Wagner, W.: Mutually consistent thermodynamic potentials for fluid water, ice and seawater: a new standard for oceanography, Ocean Sci., 4, 275-291, 2008 b, http://www.ocean-sci.net/4/275/2008/.

Fofonoff, N. P.: Physical properties of sea-water, in: The Sea, edited by: Hill, M. N., Wiley, New York, 3-30, 1962.

Fofonoff, N. P. and Millard, R. C.: Algorithms for the computation of fundamental properties of seawater, Unesco techn. pap. mar. sci., 44, online available at: http://unesdoc.unesco.org/images/ 0005/000598/059832EB.pdf, 1983.

Gatley, D. P.: Understanding Psychrometrics, 2nd ed., American Society of Heating, Refrigerating and Air-Conditioning ASHRAE, Atlanta, 2005.

Giacomo, P.: Equation for the determination of the density of moist air (1981), Metrologia, 18, 33-40, 1982.

Gibbs, J. W.: Graphical methods in the thermodynamics of fluids, Trans. Connecticut Acad. Arts Sci., 2, 309-342, 1873.

Gill, A. E.: Atmosphere Ocean Dynamics, Academic Press, San Diego, 1982.

Glansdorff, P. and Prigogine, I.: Thermodynamic Theory of Structure, Stability, and Fluctuations, Wiley Interscience, New York, 1971.

Glasstone, S.: Thermodynamics for Chemists, D. van Nostrand Company, Inc., Princeton, NJ, USA, 1947.

Goldberg, R. N. and Weir, R. D.: Conversion of temperatures and thermodynamic properties to the basis of the International Temperature Scale of 1990, Pure Appl. Chem., 64, 1545-1562, 1992.

Goodstein, D. L.: States of matter, Prentice-Hall, Inc., Englewood Cliffs, New Jersey, 1975.

Guggenheim, E. A.: Thermodynamics. An advanced treatment for chemists and physicists, North-Holland Physics Publishing, Amsterdam, 1967.

Hagen, E., Zülicke, Ch., and Feistel, R.: Near surface structures in the Cape Ghir filament off Morocco, Oceanol. Acta, 19, 577597, 1996.

Hantel, M. and Mayer, P.: Skriptum Theoretische Meteorologie I. Wiener Meteorologische Schriften, Heft 3, Facultas Universitätsverlag, Wien, 2006.

Harvey, A. H. and Huang, P. H.: First-Principles Calculation of the Air-Water Second Virial Coefficient, Int. J. Thermophys., 28, 556-565, 2007.

Helland-Hansen, B.: The Ocean Waters. International Review on Hydrobiological Hydrographic, Hydrog. Supp. Ser. 1 No. 2, Leipzig, 1912.
Hellmuth, O.: Columnar modelling of nucleation burst evolution in the convective boundary layer - first results from a feasibility study Part I: Modelling approach, Atmos. Chem. Phys., 6, 41754214, 2006,

http://www.atmos-chem-phys.net/6/4175/2006/.

Helmholtz, H. v.: Ueber atmosphaerische Bewegungen, Sitz. ber. Königl. Preuss. Akad. Wiss. Berlin, 46, 647-663, online available at: http://bibliothek.bbaw.de/bibliothek-digital/ digitalequellen/schriften/anzeige/index_html?band=10-sitz/ 1888-1 $\backslash$ \&seite: int $=665,1888$.

Henry, P. S. H.: The Specific Heats of Air, Oxygen, Nitrogen from $20^{\circ} \mathrm{C}$ to $370^{\circ} \mathrm{C}$, Proc. Roy. Soc. London, A133, 492-506, 1931.

Herrmann, S., Kretzschmar, H.-J., and Gatley, D. P.: Thermodynamic Properties of Real Moist Air, Dry Air, Steam, Water, and Ice, ASHRAE RP-1485, American Society of Heating, Refrigeration, and Air-Conditioning Engineers, Inc., Atlanta, GA, online available at: http://www.ashrae.org, 2009a.

Herrmann, S., Kretzschmar, H.-J., and Gatley, D. P.: Thermodynamic Properties of Real Moist Air, Dry Air, Steam, Water, and Ice, HVAC\&R Research, 15, 961-986, 2009b.

Herrmann, S., Kretzschmar, H.-J., Teske, V., Vogel, E., Ulbig, P., Span, R., and Gatley, D. P.: Determination of Thermodynamic and Transport Properties of Humid Air for Power-Cycle Calculations, Report PTB-CP-3, Physikalisch-Technische Bundesanstalt, Braunschweig und Berlin, Germany, 2009c.

Herrmann, S., Kretzschmar, H.-J., Teske, V., Vogel, E., Ulbig, P., Span, R., and Gatley, D. P.: Properties of Humid Air for Calculating Power Cycles, J. Eng. Gas Turbines Power, in press, 2010.

Higashi, K., Nakamura, K., and Hara, R.: The specific gravities and the vapour pressures of the concentrated sea water at $0^{\circ}-17^{\circ} \mathrm{C}$ (in Japanese), J. Soc. Chem. Ind. Japan, 34, 166-172, 1931.

Hofmann, G.: Die Thermodynamik der Taubildung, Ber. Deutsch. Wetterd., 18, 1-45, 1955.

Hofmann, G.: Verdunstung und Tau als Glieder des Wärmehaushalts, Planta, 47, 303-322, 1956.

Hyland, R. W. and Wexler, A.: The Enhancement of Water Vapor in Carbon Dioxide-Free Air at 30,40 , and $50^{\circ} \mathrm{C}$, J. Res. NBS, 77A, 115-131, 1973.

Hyland, R. W: A Correlation for the Second Interaction Virial Coefficients and Enhancement Factors for Humid Air, J. Res. NBS, 79A, 551-560, 1975.

Hyland, R. W. and Wexler, A.: Formulations for the Thermodynamic Properties of the Saturated Phases of $\mathrm{H}_{2} \mathrm{O}$ From $173.15 \mathrm{~K}$ to $473.15 \mathrm{~K}$, ASHRAE Transact., 89, 500-519, 1983a.

Hyland, R. W. and Wexler, A.: Formulations for the thermodynamic properties of dry air from $173.15 \mathrm{~K}$ to $473.15 \mathrm{~K}$, and of saturated moist air from $173.15 \mathrm{~K}$ to $372.15 \mathrm{~K}$, at pressures up to $5 \mathrm{MPa}$, ASHRAE Transact., 89, 520-535, 1983 b.

IAPWS: Guideline on the Use of Fundamental Physical Constants and Basic Constants of Water. The International Association for the Properties of Water and Steam. Gaithersburg, Mayland, USA, September 2001, Revision July 2005, online available at: http: //www.iapws.org, 2005.

IAPWS: Guideline on the Henry's Constant and Vapor-Liquid Distribution Constant for Gases in $\mathrm{H}_{2} \mathrm{O}$ and $\mathrm{D}_{2} \mathrm{O}$ at High Temperatures. The International Association for the Properties of Water and Steam, 2004, online available at: http://www.iapws.org, 2004.

IAPWS: Release on the IAPWS Formulation 2008 for the Thermo- 
dynamic Properties of Seawater. The International Association for the Properties of Water and Steam. Berlin, Germany, September 2008, online available at: http://www.iapws.org, 2008a.

IAPWS: Revised Release on the Pressure along the Melting and Sublimation Curves of Ordinary Water Substance. The International Association of the Properties of Water and Steam, Berlin, Germany, September 2008, onlien available at: http://www. iapws.org, 2008b.

IAPWS: Revised Release on the IAPWS Formulation 1995 for the Thermodynamic Properties of Ordinary Water Substance for General and Scientific Use. The International Association for the Properties of Water and Steam. Doorwerth, The Netherlands, September 2009, online available at: http://www.iapws. org, 2009a.

IAPWS: Revised Release on the Equation of State 2006 for $\mathrm{H}_{2} \mathrm{O}$ Ice Ih. The International Association for the Properties of Water and Steam, Doorwerth, The Netherlands, September 2009, online available at: http://www.iapws.org, 2009b.

IAPWS: Guideline on an Equation of State for Humid Air in Contact with Seawater and Ice, Consistent with the IAPWS Formulation 2008 for the Thermodynamic Properties of Seawater. The International Association for the Properties of Water and Steam. Niagara Falls, Canada, July 2010, in preparation, 2010.

IOC: Resolution XXV-7, INTERNATIONAL THERMODYNAMIC EQUATION OF SEAWATER (TEOS-10), INTERGOVERNMENTAL OCEANOGRAPHIC COMMISSION (of UNESCO), Twenty-fifth Session of the Assembly, Paris, 16-25 June 2009.

IOC: The international thermodynamic equation of seawater - 2010: Calculation and use of thermodynamic properties, Intergovernmental Oceanographic Commission, Manuals and Guides, UNESCO (English), online available at: http://www.TEOS-10.org), in preparation, 2010.

IUPAC: Compendium of Chemical Terminology, 2nd ed. (the "Gold Book"), compiled by: A. D. McNaught and A. Wilkinsonm, Blackwell Scientific Publications, Oxford 1997, XML on-line corrected version: http://goldbook.iupac.org (2006) created by: Nic, M., Jirat, J., and Kosata, B., updates compiled by: Jenkins, A., ISBN 0-9678550-9-8, doi:10.1351/goldbook, 1997.

Jacobsen, R. T., Clarke, W. P., Penoncello, S. G., and McCarthy, R. D.: A Thermodynamic Property Formulation for Air. I. SinglePhase Equation of State from 60 to $873 \mathrm{~K}$ at Pressures to $70 \mathrm{MPa}$, Int. J. Thermophys., 11, 169-177, 1990.

Jacobson, M. Z.: Fundamentals of Atmospheric Modeling, 2nd Edition, University Press, Cambridge, 2005.

Kammer, H. and Schwabe, K.: Einführung in die statistische Thermodynamik, Akademie-Verlag, Berlin, 1971.

Kennish, M. J.: Practical Handbook of Marine Science, CRC Press, Boca Raton, 2001.

Kittel, C.: Thermal Physics. John Wiley \& Sons, Inc., New York, 1969.

Köhler, H.: The nucleus in and the growth of hygroscopic droplets, T. Faraday Soc., 43, 1152-1161, 1936.

Korolev, A. and Isaac, G. A.: Relative humidity in liquid, mixedphase, and ice clouds, J. Atmos. Sci., 63, 2865-2880, 2006.

Kraus, E. B.: Atmosphere-Ocean Interaction, Oxford University Press, London, New York, 1972.

Landau, L. D. and Lifschitz, I. M.: Hydrodynamik, AkademieVerlag, Berlin, 1974.
Landau, L. D. and Lifschitz, I. M.: Statistische Physik, AkademieVerlag, Berlin, 1987.

Lehmann, H. P., Fuentes-Arderiu, X., and Bertello, L. F.: Glossary of terms in quantities and units in Clinical Chemistry (IUPACIFCC Recommendations 1996), Pure Appl. Chem., 68, 9571000, 1996.

Lemmon, E. W., Jacobsen, R. T., Penoncello, S. G., and Friend, D. G.: Thermodynamic Properties of Air and Mixtures of Nitrogen, Argon and Oxygen From 60 to $2000 \mathrm{~K}$ at Pressures to $2000 \mathrm{MPa}$, J. Phys. Chem. Ref. Data, 29, 331-362, 2000.

Lewis, G. L.: The osmotic pressure of concentrated solutions, and the laws of the perfect solution, J. Am. Chem. Soc., 30, 668-683, 1908.

Linke, F. and Baur, F.: Meteorologisches Taschenbuch, Geest \& Portig, Leipzig, 1970.

List, R. J.: Smithsonian Meteorological Tables, Smithson. Misc. Collect. 114, Smithson. Inst., Washington DC, 1951.

Lunt, D. J., Foster, G. L., Haywood, A. M., and Stone, E. J.: Late Pliocene Greenland glaciation controlled by a decline in atmospheric $\mathrm{CO}_{2}$ levels, Nature, 454, 1102-1105, 2008.

Magee, J. W.: Molar Heat Capacity at Constant Volume for Air from 67 to $300 \mathrm{~K}$ at Pressures to $35 \mathrm{MPa}$, Int. J. Thermophys., 15, 849-861, 1994.

Margenau, H. and Murphy, G. M.: The Mathematics of Physics and Chemistry, D. van Nostrand Company, Inc., New York, 1943.

Marion, G. M., Millero, F. J., and Feistel, R.: Precipitation of solid phase calcium carbonates and their effect on application of seawater $S_{A} T$ P models, Ocean Sci., 5, 285-291, 2009, http://www.ocean-sci.net/5/285/2009/.

McDougall, T. J.: Potential Enthalpy: A conservative oceanic variable for evaluating heat content and heat fluxes, J. Phys. Oceanogr., 33, 945-963, 2003.

McDougall, T. J. and Feistel, R.: What Causes the Adiabatic Lapse Rate?, Deep-Sea Res. I, 50, 1523-1535, 2003.

Millero, F. J.: The thermodynamics of seawater. Part II. Thermochemical properties, Ocean Sci. Eng., 8, 1-40, 1983.

Millero, F. J.: Physical Chemistry of Natural Waters, WileyInterscience, New York, 2001.

Millero, F. J.: Chemical Oceanography, CRC Press, Boca Raton, 1996.

Millero, F. J., Feistel, R., Wright, D. G., and McDougall, T. J.: The composition of Standard Seawater and the definition of the Reference-Composition Salinity Scale, Deep-Sea Res. I, 55, 5072, 2008.

Millero, F. J. and Huang, F.: The density of seawater as a function of salinity ( 5 to $70 \mathrm{~g} \mathrm{~kg}^{-1}$ ) and temperature ( 273.15 to 363.15 K), Ocean Sci., 5, 91-100, 2009, http://www.ocean-sci.net/5/91/2009/.

Millero, F. J. and Leung, W. H.: The thermodynamics of seawater at one atmosphere, Am. J. Sci., 276, 1035-1077, 1976.

Möbius, H.-H. and Dürselen, W.: Chemische Thermodynamik, VEB Deutscher Verlag für Grundstoffindustrie, Leipzig, 1973.

Montgomery, R. B.: Observations of vertical humidity distribution above the ocean surface and their relation to evaporation, Pap. Phys. Oceanogr. Meteor., 7, 1-30, 1940.

Morris, V., Clemente-Colón, P., Nalli, N. R., Joseph, E., Armstrong, R. A., Detrés, Y., Goldberg, M. D., Minnett, P. J., and Rick Lumpkin, R.: Measuring Trans-Atlantic Aerosol Transport From Africa, Eos, 87, 565-571, 2006. 
Nalli, N. R., Clemente-Colon, P., Morris, V., Joseph, E., Szczodrak, M., Minnett, P. J., Shannahoff, J., Goldberg, M. D., Barnet, C., Wolf, W. W., Feltz, W. F., and Knuteson, R. O.: Profile observations of the Saharan air layer during AEROSE 2004, Geophys. Res. Lett., 32, L05815, 1-5, 2005.

O'Dowd, C. D., Smith, M. H., Consterdine, I. E., and Lowe, J. A.: Marine aerosol, sea-salt, and the marine sulphur cycle: A short review, Atmos. Environ., 31, 73-80, 1997.

Ostwald, W.: Lehrbuch der Allgemeinen Chemie, Leipzig, 1896.

Panin, G. N. and Brezgunov, V. S.: Influence of the Salinity of Water on its Evaporation, Izvestiya, Atmos. Ocean. Phys., 43, 663-665, 2007.

Pauluis, O., Czaja, A., and Korty, R.: The Global Atmospheric Circulation on Moist Isentropes, Science, 321, 1075-1078, 2008.

Picard, A., Davis, R. S., Gläser, M., and Fujii, K.: Revised formula for the density of moist air (CIPM-2007), Metrologia, 45, 149$155,2008$.

Pollitzer, F. and Strebel, E.: Over the Influence of Indifferent Gases on the Saturation Steam Concentration of Liquids, Z. Phys. Chem., 110, 768-785, 1924 (in German).

Preston-Thomas, H.: The international temperature scale of 1990 (ITS-90), Metrologia, 27, 3-10, 1990.

Prigogine, I. and Defay, R.: Chemical Thermodynamics, Longmans Green, London, 1954.

Pruppacher, H. R. and Klett, J. D.: Microphysics of Clouds and Precipitation. Second Revised and Enlarged Edition with an Introduction to Cloud Chemistry and Cloud Electricity, Kluwer Academic Publishers, Dordrecht/Boston/London, 1997.

Reif, F.: Fundamentals of Statistical and Thermal Physics, McGraw-Hill Book Co., Singapore, 1965.

Robinson, R. A.: The vapour pressure and osmotic equivalence of sea water, J. Mar. Biol. Ass. United Kingdom, 33, 449-455, 1954.

Rogers, R. R. and Yau, M. K.: Short Course in Cloud Physics, Third Edition, Butterworth-Heinemann, Oxford, 1989.

Safarov, J., Millero, F., Feistel, R., Heintz, A., and Hassel, E.: Thermodynamic properties of standard seawater: extensions to high temperatures and pressures, Ocean Sci., 5, 235-246, 2009, http://www.ocean-sci.net/5/235/2009/.

Santer, B. D., Mears, C., Wentz, F. J., Taylor, K. E., Gleckler, P. J., Wigley, T. M. L., Barnett, T. P., Boyle, J. S., Brüggemann, W., Gillett, N. P., Klein, S. A., Meehl, G. A., Nozawa, T., Pierce, D. W., Stott, P. A., Washington, W. M., and Wehner, M. F.: Identification of human-induced changes in atmospheric moisture content, P. Natl. Acad. Sci. USA, 104, 15248-15253, 2007.

Schmelzer, J. and Schweitzer, F.: Ostwald ripening of bubbles in liquid-gas solutions, J. Non-Equilib. Thermodyn., 12, 255-270, 1987.

Shaw, A. N.: The Derivation of Thermodynamical Relations for a Simple System, Philos. T. Roy. Soc. London A, 234, 299-328, 1935.

Seinfeld, J. H. and Pandis, S. N.: Atmospheric Chemistry \& Physics: From Air Pollution to Climate Change, John Wiley \& Sons, New York, 1998.

Siedler, G. and Peters, H.: Properties of Sea Water: Physical Properties, in: Oceanography, edited by: Sündermann, J., LandoltBörnstein V/3a, Springer, Berlin, Heidelberg, 1986.

Smith, S. D.: Coefficients for the sea surface wind stress, heat flux, and wind profiles as a function of wind speed and temperature, $\mathrm{J}$.
Geophys. Res., 93(C12), 15467-15472, 1988.

Sonntag, D.: Formeln verschiedenen Genauigkeitsgrades zur Berechnung des Sättigungsdampfdruckes über Wasser und über Eis und ihre Anwendung auf einige praktische Feuchtemeßaufgaben, Abh. Met. Dienst. DDR, 17, 1-59, 1982.

Sonntag, D.: Important new Values of the Physical Constants of 1986, Vapour Pressure Formulations based on the ITS-90, and Psychrometer Formulae, Z. Meteorol., 40, 340-344, 1990.

Sonntag, D.: The History of Formulations and Measurements of Saturated Water Vapour Pressure. Third International Symposium on Humidity and Moisture, National Physical Laboratory, Teddington, Middlesex, UK, April 1998, Vol. 1, 93-102, 1998.

Span, R., Lemmon, E. W., Jacobsen, R. T., Wagner, W., and Yokozeki, A.: A Reference Equation of State for the Thermodynamic Properties of Nitrogen for Temperatures from 63.151 to $1000 \mathrm{~K}$ and Pressures to $2200 \mathrm{MPa}$, J. Phys. Chem. Ref. Data, 29, 1361-1433, 2000.

Stanley, E. H.: Introduction to Phase Transitions and Critical Phenomena, Clarendon Press, Oxford, 1971.

Stewart, R. B., Jacobsen, R. T., and Wagner, W.: Thermodynamic Properties of Oxygen from the Triple Point to $300 \mathrm{~K}$ with Pressures to $80 \mathrm{MPa}$, J. Phys. Chem. Ref. Data, 20, 917-1021, 1991.

Stott, R. A., Sutton, R. T., and Smith, D. M.: Detection and attribution of Atlantic salinity changes, Geophys. Res. Lett., 35, L21702, doi:10.1029/2008GL035874, 2008.

Stull, R. B.: An Introduction to Boundary Layer Meteorology, Kluwer Academic Publishers, Dordrecht, The Netherlands, 2003.

Sun, R., Hu, W., and Duan, Z.: Prediction of Nitrogen Solubility in Pure Water and Aqueous $\mathrm{NaCl}$ Solutions up to High Temperature, Pressure, and Ionic Strength, J. Solution Chem., 30, 561573, 2004.

Tegeler, Ch., Span, R., and Wagner, W.: A New Equation of State for Argon Covering the Fluid Region for Temperatures From the Melting Line to $700 \mathrm{~K}$ at Pressures up to $1000 \mathrm{MPa}$, J. Phys. Chem. Ref. Data, 28, 779-850, 1999.

Tetens, O.: Über einige meteorologische Begriffe, Z. Geophys., 6, 297-309, 1930.

Tillner-Roth, R.: Fundamental Equations of State, Shaker Verlag, Aachen, 1998.

Trenberth, K. E., Caron, J. M., and Stepaniak, D. P.: The atmospheric energy budget and implications for surface fluxes and ocean heat transports, Clim. Dynam., 17, 259-276, 2001.

Unesco: The Practical Salinity Scale 1978 and the International Equation of State of Seawater 1980, Unesco techn. pap. mar. sci., 36, 8-25, online available at: http://unesdoc.unesco.org/images/ 0004/000461/046148eb.pdf, 1981.

Van Wylen, G. J. and Sonntag, R. E.: Fundamentals of Classical Thermodynamics, John Wiley \& Sons, Inc., New York, 1965.

Wagner, W. and de Reuck, M.: International thermodynamic tables of the fluid state-9, oxygen, Blackwell Scientific, Oxford, 1987.

Wagner, W. and Kretzschmar, H.-J.: International Steam Tables, 2nd edition. Springer Verlag, Berlin, Heidelberg, 2008.

Wagner, W. and Pruß, A.: The IAPWS formulation 1995 for the thermodynamic properties of ordinary water substance for general and scientific use, J. Phys. Chem. Ref. Data, 31, 387-535, 2002.

Walton Smith, F. G.: Handbook of Marine Science, Vol. I. CRC Press, Cleveland, 1974. 
Weare, B. C., Strub, P. T., and Samuel, M. D.: Annual mean surface heat fluxes in the tropical Pacific Ocean, J. Phys. Oceanogr., 2, 705-717, 1981.

Webster, T. J.: The Effect on Water Vapour Pressure of Superimposed Air Pressure, J. Soc. Chem. Ind. London, 69, 343-346, 1950.

Weiss, R. F.: The solubility of nitrogen, oxygen and argon in water and seawater, Deep-Sea Res., 17, 721-735, 1970.

Weiss, R. F. and Price, B. A.: Nitrous oxide solubility in water and seawater, Mar. Chem., 8, 347-359, 1980.

Weller, R. A., Bradley, E. F., Edson, J. B., Fairall, C. W., Brooks, I., Yelland, M. J., and Pascal, R. W.: Sensors for physical fluxes at the sea surface: energy, heat, water, salt, Ocean Sci., 4, 247-263, 2008 , http://www.ocean-sci.net/4/247/2008/.

Wells, N. C. and King-Hele, S.: Parametrization of tropical ocean heat flux, Q. J. Roy. Meteorol. Soc., 116, 1213-1224, 1990.

Wieser, M. E.: Atomic Weights of the elements (IUPAC Technical Report), Pure Appl. Chem., 78, 2051-2066, online available at: http://www.iupac.org/publications/pac/78/11/2051/, 2006.
Willett, K. M., Gillett, N. P., Jones, P. D., and Thorne, P. W.: Attribution of observed surface humidity changes to human influence, Nature, 44(9), 710-712, 2007.

Witting, R.: Untersuchungen zur Kenntnis der Wasserbewegungen und der Wasserumsetzung in den Finnland umgebenden Meeren, I, Der Bottnische Meerbusen in den Jahren 1904 und 1905, Erster Teil, Finnl. Hydrogr.-Biol. Unters., 2, 1-246, 1908.

Woolley, H. W.: Thermodynamic properties for $\mathrm{H}_{2} \mathrm{O}$ in the ideal gas state, in: Water and Steam, edited by: Straub, J. and Scheffler, K., Proceedings of the 9th International Conference on the Properties of Steam, München, 1979, Pergamon Press, Oxford-New YorkToronto-Sydney-Paris-Frankfurt, 166-175, 1980.

Wright, D. G., Feistel, R., Jackett, D. R., Miyagawa, K., Reissmann, J. H., Wagner, W., Overhoff, U., Guder, C., Tchijov, V., Feistel, A., and Marion, G. M.: Numerical implementation and oceanographic application of the thermodynamic potentials of water, vapour, ice, seawater and air. Part II: The Library Routines, Ocean Sci., submitted, 2009.

Wylie, R. G. and Fisher, R. S.: Molecular Interaction of Water Vapor and Air, J. Chem. Eng. Data, 41, 133-142, 1996. 